\title{
The redesign and implementation of a reliable robotic pipe inspection system
}

\author{
A thesis \\ submitted in fulfilment \\ of the requirements for a Degree \\ of \\ Master of Engineering in Electronic \\ and Computer Systems Engineering \\ at \\ Victoria University of Wellington
}

by

Stephen Winch

TE WHARE WĀNANGa O TE ŪPOKo O TE IKA A MĀUI

Victoria University of Wellington 



\begin{abstract}
Robotic units used in pipe inspection are expensive to purchase and repair. Many companies do not offer long term support for the repair and maintenance of their robotic units.

Consequently Associated Environmental Services Limited (AES), a Wellington based pipe inspection company approached Victoria University to facilitate the repair of a non-functional system.

As a result this Masters project aims to design and implement a reliable pipe inspection system for use by AES. This thesis outlines and discusses the circuit boards and software designed for such a robotic unit. A reliable graphical user interface and internal circuitry for the robot was designed and is also presented.
\end{abstract}





\section{Acknowledgements}

There are many people other than myself that made the completion of this project possible, first and foremost I would like to thank Bryan Pobar and Prof. Dale Carnegie.

To Bryan, I would like to thank you for allowing me to work on this project for your company. I would also like to thank you for your financial support. Without either of these I doubt I would have started, let alone completed a Masters project.

To Dale, thank you for accepting me as one of your postgraduate students two years running, without your support I would not have considered doing Masters.

To the electronic technicians Jason Edwards and Tim Exley, and the workshop technicians Alan Rennie, Manu Pouajen-Blakiston and Nick Grinter, thank you for your expertise, knowledge and help in making this project possible.

To my family thank you for your support and understanding, and attempts at comprehension.

Finally I would like to thank you for reading this Thesis. I hope you find it as informative and interesting as I did when creating it. 



\section{Contents}

1 Introduction xvii

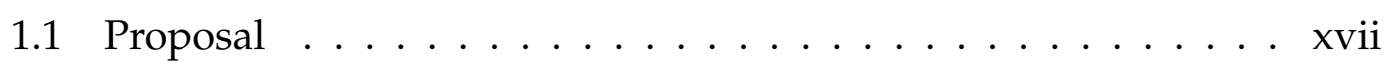

1.2 Project Specifications . . . . . . . . . . . . . xix

1.3 Project Milestones . . . . . . . . . . . . . . . . xx

1.4 Thesis Structure . . . . . . . . . . . . . . . xxi

2 Background 1

2.1 Current Robotic Systems . . . . . . . . . . . . . . . . . 1

2.1 .1 IBAK Robots . . . . . . . . . . . . . . . . . . . 1

2.1.2 Radiodetection PearPoint ............. 6

2.1 .3 Rovvers...................... 9

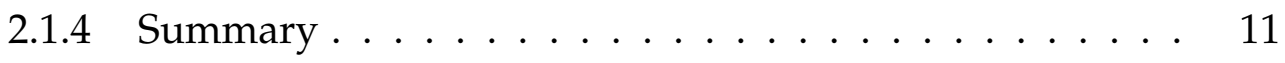

2.2 The Non-Functional Robotic Unit . . . . . . . . . . . . . . . 13

2.3 Previous Work . . . . . . . . . . . . . . . . . . 16

3 Communication and Transmission $\quad 19$

3.1 Overview ............................... 19

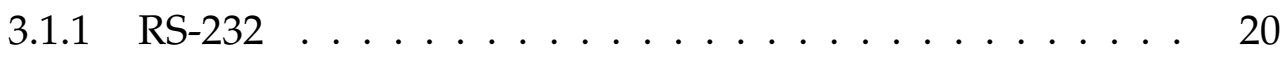

3.1 .2 RS-485 ............................ 20

3.1 .3 USB ............................... 21

3.1 .4 Firewire .......................... 22

3.1 .5 Fibre Optics ...................... 22

3.1.6 Communication Summary . . . . . . . . . . . . . 23

3.2 Video Setup and Standards . . . . . . . . . . . . . . . 24

3.2 .1 Analogue Video . . . . . . . . . . . . . . . . . 25

3.2 .2 Digital Video . . . . . . . . . . . . . . . 25 
3.2.3 Selected video comparison . . . . . . . . . . . . . 26

3.2.4 Video Configurations . . . . . . . . . . . . . . . 27

3.2.5 Video configuration summary . . . . . . . . . . 30

4 Robotic Printed Circuit Boards $\quad 31$

4.1 Camera Board . . . . . . . . . . . . . . . . . 31

4.1 Revision $1 \ldots \ldots \ldots 33$

4.1 .2 Revision $2 \ldots \ldots \ldots \ldots \ldots$

4.2 Main Control Board . . . . . . . . . . . . . . . . . . . . 36

4.2.1 Revision $1 \ldots \ldots \ldots . \ldots \ldots$

4.2 .2 Revision $2 \ldots \ldots \ldots \ldots$. . . . . . . . . . 41

4.2 .3 Revision $3 \ldots \ldots \ldots \ldots$

4.2 .4 Revision $4 \ldots \ldots \ldots . \ldots \ldots 4$

4.3 Plug Board . . . . . . . . . . . . . . . . 46

4.3 .1 Revision $1 \ldots \ldots \ldots 47$

4.3 .2 Revision $2 \ldots \ldots \ldots \ldots$. . . . . . . . . . . 49

4.4 Power Module Board . . . . . . . . . . . . . . . . . . . . . 50

4.4 .1 Revision $1 \ldots \ldots \ldots 52$

4.4 .2 Revision $2 \ldots \ldots \ldots \ldots$

4.5 Internal Mounting . . . . . . . . . . . . . . . . . . 54

4.6 Internal PCB Summary . . . . . . . . . . . . . . . . 56

5 External Printed Circuirt Boards 59

5.1 Base Station Board . . . . . . . . . . . . . . . . . 59

5.1 Revision $1 \ldots \ldots \ldots 1$

5.1 .2 Revision $2 \ldots \ldots \ldots \ldots$. . . . . . . . 62

5.1 .3 Revision $3 \ldots \ldots \ldots$................. 64

5.2 Motor Driver Board . . . . . . . . . . . . . . . . . . . 65

5.2 .1 Revision $1 \ldots \ldots \ldots 6$

5.3 Camera Test Board . . . . . . . . . . . . . . . 68

5.4 USB to Serial Board . . . . . . . . . . . . . . . . . . . . . 69

5.5 External PCB Summary . . . . . . . . . . . . . . . . . 70

6 Lights, Camera, Action $\quad 73$

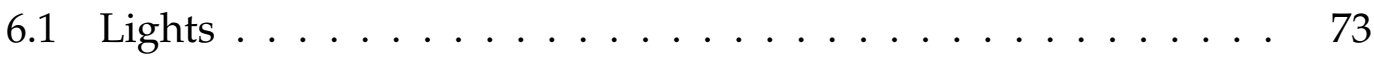


6.2 Commercial peripherals . . . . . . . . . . . . . . 75

6.2 .1 Cameras . . . . . . . . . . . . . . . . . . 75

6.2 .2 Camera Functions . . . . . . . . . . . . . . . . 78

6.2 .3 Motor Drivers . . . . . . . . . . . . . . . . . . . . . 79

6.2 .4 Joystick . . . . . . . . . . . . . . . . . . . 82

6.2 .5 Internal Motors . . . . . . . . . . . . . . . . 83

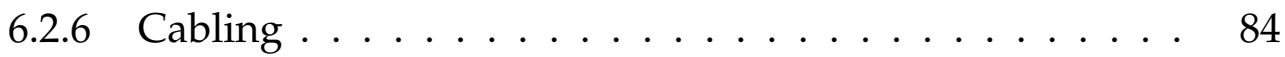

6.2 .7 Reel Motors . . . . . . . . . . . . . . . . . . . . 87

6.3 Hardware Summary . . . . . . . . . . . . . . . . . . . . . 89

$\begin{array}{lll}7 & \text { Software } & 91\end{array}$

7.1 C\# Graphical User Interface . . . . . . . . . . . . . . . . . . 92

7.1.1 Revision 1: C\# Implementation . . . . . . . . . . . . . 93

7.1.2 Revision 2: Joystick Adaptation . . . . . . . . . . . . 102

7.1.3 Revision 3: Indicators . . . . . . . . . . . . . . . . . 106

7.1.4 Revision 4: Video Recording \& Relocation . . . . . . . . 107

7.1.5 Improvements Over Ristic Version . . . . . . . . . . . . 111

7.2 Microcontroller code . . . . . . . . . . . . . . . . . . 113

7.2.1 Robot Code . . . . . . . . . . . . . . . . . . . . 113

7.2.2 Base Station Code . . . . . . . . . . . . . . . . . . . . . 114

7.3 Code Summary . . . . . . . . . . . . . . . . . . . . 116

8 Evaluation and Conclusion $\quad 117$

8.1 Evaluation . . . . . . . . . . . . . . . . . . . . . . . 117

8.1 .1 Efficiency . . . . . . . . . . . . . . . . . . . . 118

8.1 .2 Review . . . . . . . . . . . . . . . . . . . . . 119

8.2 Future Work . . . . . . . . . . . . . . . . . . . . . . . . 127

8.2 .1 Robot . . . . . . . . . . . . . . . . . . . . . . . 127

8.2.2 Base Station . . . . . . . . . . . . . . . . . . . . . . . . . . 129

8.2 .3 GUI . . . . . . . . . . . . . . . . . . . . . . . . 131

$8.2 .4 \quad$ Reel design . . . . . . . . . . . . . . . . . . . . . . 132

8.3 Summary . . . . . . . . . . . . . . . . . . . . . . 132

$\begin{array}{ll}\text { References } & 135\end{array}$

$\begin{array}{ll}\text { Appendix : CD } & 141\end{array}$ 



\section{List of Figures}

2.1 IBAK Systems $\ldots \ldots \ldots \ldots$. . . . . . . . . . . . 2

2.2 IBAK Tractor units . . . . . . . . . . . . . . 3

2.3 KRA Systems . . . . . . . . . . . . . . . . 4

2.4 IBAK Cameras . . . . . . . . . . . . . . . . . . . 5

2.5 IBAK BS5 setup . . . . . . . . . . . . . . 6

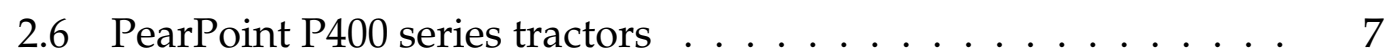

2.7 PearPoint P377 Control Centre . . . . . . . . . . . 8

2.8 PearPoint Portable System . . . . . . . . . . . . . . 9

2.9 Rovvers . . . . . . . . . . . . . . . . . . 9

2.10 Environsight modular system design . . . . . . . . . . . . 11

2.11 Project Robot . . . . . . . . . . . . . . . . . . 13

2.12 Gearing Configuration of Camera Head . . . . . . . . . . . 14

2.13 Camera pan gearing diagram . . . . . . . . . . . . . 15

2.14 SolidWorks Model of Camera Head . . . . . . . . . . . . . . . 15

2.15 Drive Motor Gear Systems . . . . . . . . . . . . . . . . 16

2.16 Mr Ristic's LabVIEW GUI . . . . . . . . . . . . . . . . 17

4.1 Camera Board Common Parts . . . . . . . . . . . . . . 32

4.2 Camera Board Common Comparators . . . . . . . . . . 33

4.3 Camera Board Revision 1 . . . . . . . . . . . . . . . . . . 34

4.4 Camera Board Revision 2 . . . . . . . . . . . . . . . . . 35

4.5 Main Control Board Microcontroller . . . . . . . . . . . . . 37

4.6 Main Control Board Common I/O . . . . . . . . . . . . 38

4.7 Main Control Board Communication . . . . . . . . . . . . . . . 38

4.8 Main Control Board Revision 1 PCB . . . . . . . . . . . . . . . 39 
4.9 Main Board Power Circuitry . . . . . . . . . . . . . . . . . . . 39

4.10 Main Board Plug Location . . . . . . . . . . . . . . . . . . 40

4.11 Main Control Board Revision 1 Schematics . . . . . . . . . . . . 41

4.12 Main Board Revision 2 Regulators . . . . . . . . . . . . . . . 42

4.13 Main Board Revision 2 Input Header . . . . . . . . . . . . . . 42

4.14 Main Board Revision 3 Plug and Play . . . . . . . . . . . . . . . 44

4.15 Wiring Inside of Robot . . . . . . . . . . . . . . . . . 46

4.16 Plug Board Revision 1 I/O Headers . . . . . . . . . . . . . . . 47

4.17 Plug Board Revision 1 I/O Plugs . . . . . . . . . . . . . . 48

4.18 Plug Board Revision 1 PCB . . . . . . . . . . . . . . . . . . 49

4.19 Main Motor in Base . . . . . . . . . . . . . . . . . . . . . . 49

4.20 Plug Board Revision $2 \ldots \ldots$. . . . . . . . . . . 50

4.21 Vicor DC-DC Converter . . . . . . . . . . . . . . . . 51

4.22 Power Module Board Revision 1 . . . . . . . . . . . . 52

4.23 header Positioning on Power Module Board . . . . . . . . . . . . 53

4.24 Power Module Board Revision 2 . . . . . . . . . . . . . . . 53

4.25 Lid with Vicor Module . . . . . . . . . . . . . . . . . . . 54

4.26 Board Stacking Results . . . . . . . . . . . . . . . 55

4.27 Camera Board in Head . . . . . . . . . . . . . . . . 55

5.1 Base Station Board Common Components . . . . . . . . . . . . . 60

5.2 Base Station Board Revision 1 Schematics . . . . . . . . . . . 61

5.3 Base Station Board Revision 1 PCB . . . . . . . . . . . . . . . 62

5.4 Base Station Board Revision $2 \ldots . . \ldots 63$

5.5 Base Station Board Revision $2 \ldots \ldots$. . . . . . . . . . . 64

5.6 Base Station Rev 3 Modification . . . . . . . . . . . . . . . 65

5.7 Custom IGBT Footprint . . . . . . . . . . . . . . . . 66

5.8 YC4469CPD Driver IC $\ldots \ldots \ldots$. . . . . . . . . . . 67

5.9 Motor Driver Revision $1 \ldots \ldots 7$

5.10 Camera Test Board . . . . . . . . . . . . . . . . . 69

5.11 USB to Serial Board . . . . . . . . . . . . . . . 70

6.1 LED Used for Lighting . . . . . . . . . . . . . . . . . 74

6.2 LED Light Socket . . . . . . . . . . . . . . . . . . . . . . 74 
6.3 LED Light Socket Drawing . . . . . . . . . . . . . . . . . . 75

6.4 Sony Camera Modules . . . . . . . . . . . . . . . . . . 76

6.5 Motors Driver Setup ． . . . . . . . . . . . . . . . . . 79

6.6 SyRen10 Motor Driver Characteristics . . . . . . . . . . . . . . 80

6.7 Motor Driver Filter . . . . . . . . . . . . . . . . . . . . 82

6.8 Logitech Joystick . . . . . . . . . . . . . . . . . . . 82

6.9 Faulhaber DC Motors and Gearboxes . . . . . . . . . . . . . . . 84

6.10 Current Reel . . . . . . . . . . . . . . . . . . . . . 87

7.1 System Hierarchy . . . . . . . . . . . . . . . . . . . . . . . . . . . 92

7.2 C\# Front Panel . . . . . . . . . . . . . . . . . . . . 93

7.3 C\# Code Window . . . . . . . . . . . . . . . . . . . . . . . 94

7.4 GUI Revision $1 \ldots \ldots$. . . . . . . . . . . . . . . 95

7.5 Camera Data Packet Code . . . . . . . . . . . . . . . . 96

7.6 Video Closure Code . . . . . . . . . . . . . . . . . . . . . . 99

7.7 Serial Port Closure Code . . . . . . . . . . . . . . . . . . 100

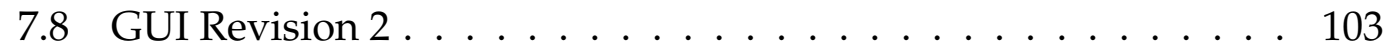

7.9 Logitech Joystick Buttons . . . . . . . . . . . . . . . . . . . . . 104

7.10 GUI Revision $3 \ldots$. . . . . . . . . . . . . . . . . . 106

7.11 Uncompressed Video Size . . . . . . . . . . . . . . 107

7.12 Video Compression Comparison . . . . . . . . . . . . . 109

7.13 GUI Revision $4 \ldots$. . . . . . . . . . . . . . . . 110

7.14 Taut Sensor Code . . . . . . . . . . . . . . . . . . . . . . . 114

8.1 Main Control Board Regulator Efficiency _ . . . . . . . . . . 118

8.2 Camera Board Regulator Efficiency . . . . . . . . . . . . . . . 119

8.3 Final Main Control Board . . . . . . . . . . . . . . . . . 120

8.4 Final Plug Board . . . . . . . . . . . . . . . . . 121

8.5 Final Power Module Board . . . . . . . . . . . . . . . . . . . . 121

8.6 Final Camera Control Boards . . . . . . . . . . . . . . . . 122

8.7 Hardware Over Rotation Prevention . . . . . . . . . . . . . . . 123

8.8 Final Base Station Board . . . . . . . . . . . . . . . . . . . . 124

8.9 Final Motor Driver . . . . . . . . . . . . . . . . . . . 125

8.10 Exploded View of Robotic Unit . . . . . . . . . . . . . . . 128 



\section{List of Tables}

2.1 IBAK Camera Comparison . . . . . . . . . . . . . . . . 5

2.2 Ideal Robotic System Requirements . . . . . . . . . . . . . . 12

3.1 ATSC Digital Video Standards . . . . . . . . . . . . . . . 26

3.2 Analogue and Digital Video Standards . . . . . . . . . . . 26

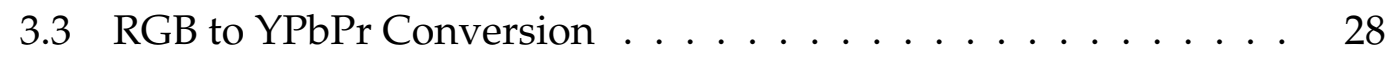

6.1 Camera Command List . . . . . . . . . . . . . . . . . . . 78

6.2 Motor Driver DIP Configurations . . . . . . . . . . . . . . 81

7.1 Camera Control Strings . . . . . . . . . . . . . . . . . . . . . 101

7.2 Daily Video File Size . . . . . . . . . . . . . . . . . 110

8.1 Project Versus Ideal . . . . . . . . . . . . . . . . . . 126 



\section{Chapter 1}

\section{Introduction}

\subsection{Proposal}

In the pipe inspection industry it is necessary to have up-to-date equipment, with low maintenance costs in order to be profitable. Current robotic pipe inspection systems cost a minimum of NZD $\$ 150,000$ with repairs costing in the tens of thousands [1].

Associated Environmental Services (AES), the company for whom this project has been undertaken in association with, would like to expand their business further into the New Zealand market. Currently they operate solely in the Wellington region but with the ability to self repair their systems they believe that they will be able to expand into the Auckland and Christchurch regions. These three regions contain approximately $26,000 \mathrm{~km}$ of sewerage and storm water piping; Auckland dominates this with over 22,000 km of piping alone [2]-[4]. Without adequate inspection of the pipes, faults can go un-noticed until a serious leakage occurs, polluting the environment and/or disrupting day-today activities.

In the case of waste water (sewerage) pipes an intrusion into the pipes will 
cause an extrusion of waste into the environment contaminating the region. Identifying problem areas before serious damage occurs is a key factor in keeping the environment clean.

Damage to storm water pipes can cause flooding during later storms due to root penetration causing blockages and infrastructure damage when pipes open in sections causing ground levels to shift. This can be especially serious as storm water pipes run parallel and under roadways.

AES own two non-functional Optimess pipe inspection robots that are no longer supported by the manufacturer. The current operational robotic units used are PearPoint units designed and built by the British company Radio Detection Ltd[5]. Repair options for these units are currently restricted to sending the units back to Britain as no repair facility exists locally. This is an expensive and time consuming operation. Since they use custom components in all of their robots, servicing by AES technicians is not an option.

The robots considered in this project are the two non-functioning robots from the Germany company Optimess. The smaller of these robots is designed to inspect pipes from $150 \mathrm{~mm}$ in diameter and weighs $20 \mathrm{~kg}$, the other is designed to inspect pipes from $300 \mathrm{~mm}$ in diameter and weighs $60 \mathrm{~kg}$. Initially the smaller robot of the two is considered after consultation with Bryan Pobar of AES [1]. This unit has a solid body with a swivelling camera head which is explain in detail in section 2.2 .

The robot operates at the end of a 200 to 300 metre long cable. When the robot is inserted into a pipe, communication between the laptop control station and the robotic unit is via this cable. Wireless communication is not required as the robot needs a power supply which is also provided via this cable that can easily support transmission wires. The robot is unable to internally carry its own power supply due to its small dimensions dictated by the size of the pipes 
it must inspect.

The non-support of older robotic units, a scarcity of repair options coupled with the company's desire to be a competitor on the national scale provided the motivation of this project. The goal is to design inexpensive hardware and software repair and upgrade solutions for the AES robots and control stations. This includes a software interface that can be easily used by the field technicians. The ideal outcome for AES would be for the robots to be able to be repaired by a person who has minimal training in electronics.

\subsection{Project Specifications}

The specific objective of this project is to take the system started by a previous Victoria University student, Aleksandar Ristic, and progress the system such that a robust and reliable system will be able to be completed at a future date. Four important criteria provided by AES are that the system should be robust, reliable, user friendly and extendable.

The system must be user friendly in both operation and repairs so that significant retraining of the field technicians in how to use the system is not required as well as allowing for repairs of the system to be made by a person with limited knowledge in electronics. Additionally, replacement components should be easily and inexpensively available.

The objective of making this system extendable is so that it is possible to use the hardware and software of this project as a platform for creating a working system with the larger non-functional robot. 


\subsection{Project Milestones}

To meet the specifications set by AES, the following milestones were set:

The first task in this project is to research current systems on the market so that a system that is able to compete with these may be designed.

Secondly, the circuitry and coding designed by Mr. Ristic needs to be understood.

Thirdly, the problems with the existing circuitry need to be found so that robust and reliable circuit boards can be created.

Fourthly, external peripherals required to upgrade this system to current standards require sourcing of components as malfunctions or otherwise obsolete components should be identified and replaced.

Fifthly, the user interface needs to be designed to allow for user friendly control of the system.

The sixth milestone is testing, redesigning and evaluation of the system so that a decision can be made on how to proceed with the project.

The final result of the system needs to be a significant step forward in the design of a robust and reliable pipe inspection system. Internal circuitry should be designed and external components specified and sourced where possible. Note the scope of this project is not to fully design the external units since this is a significant task and neither sufficient time nor resources are available. 


\subsection{Thesis Structure}

In Chapter 2 an introduction into current robotic pipe inspection systems is presented with emphasis being placed on their overall specifications so that a competitive pipe inspection system is able to be designed for AES. A brief introduction into the robotic unit used in this project and the work carried out by Ristic is also discussed.

In Chapter 3 the relevant communication protocols and the different types of video transmission is explained.

Chapters 4 and 5 cover the design of all Printed Circuit Boards (PCBs) in this project as well as why certain components and methods were chosen over others.

Chapter 6 covers the commercially obtained hardware peripherals used in this project.

Chapter 7 details the design and implementation of the code for the control interface required in this project including why one application was chosen over another and how each section of the program was implemented. The chapter also includes code written for the microcontrollers present on two of the PCBs.

Chapter 8 gives results of this project, concludes this thesis by reviewing the project achievements and discusses potential future additions required to make this system fully operational. 



\section{Chapter 2}

\section{Background}

\subsection{Current Robotic Systems}

Before the design and implementation of a reliable pipe inspection system can be undertaken, research of current systems available and their specifications needs to be carried out so that the redesigned system has similar or superior specifications at a lower maintenance cost. Currently two of the main suppliers of CCTV pipe inspection equipment are IBAK and Radiodetection, although other systems, such as the Rovvers (section 2.1.3) exist.

\subsubsection{IBAK Robots}

IBAK was founded in late September of 1945 as "Ingenieurbüro Atlas Kiel" and is one of the biggest manufacturers of CCTV inspection equipment in the world[6]. Based in Kiel, Germany, IBAK has a selection of 4 robotic units from their custom designed modular systems. These systems are separated into four sections; the first section requires the selection of the robotic tractor unit; the second section specifies the camera component. In the third section the reel required is selected. The fourth section involves selecting the integrated 


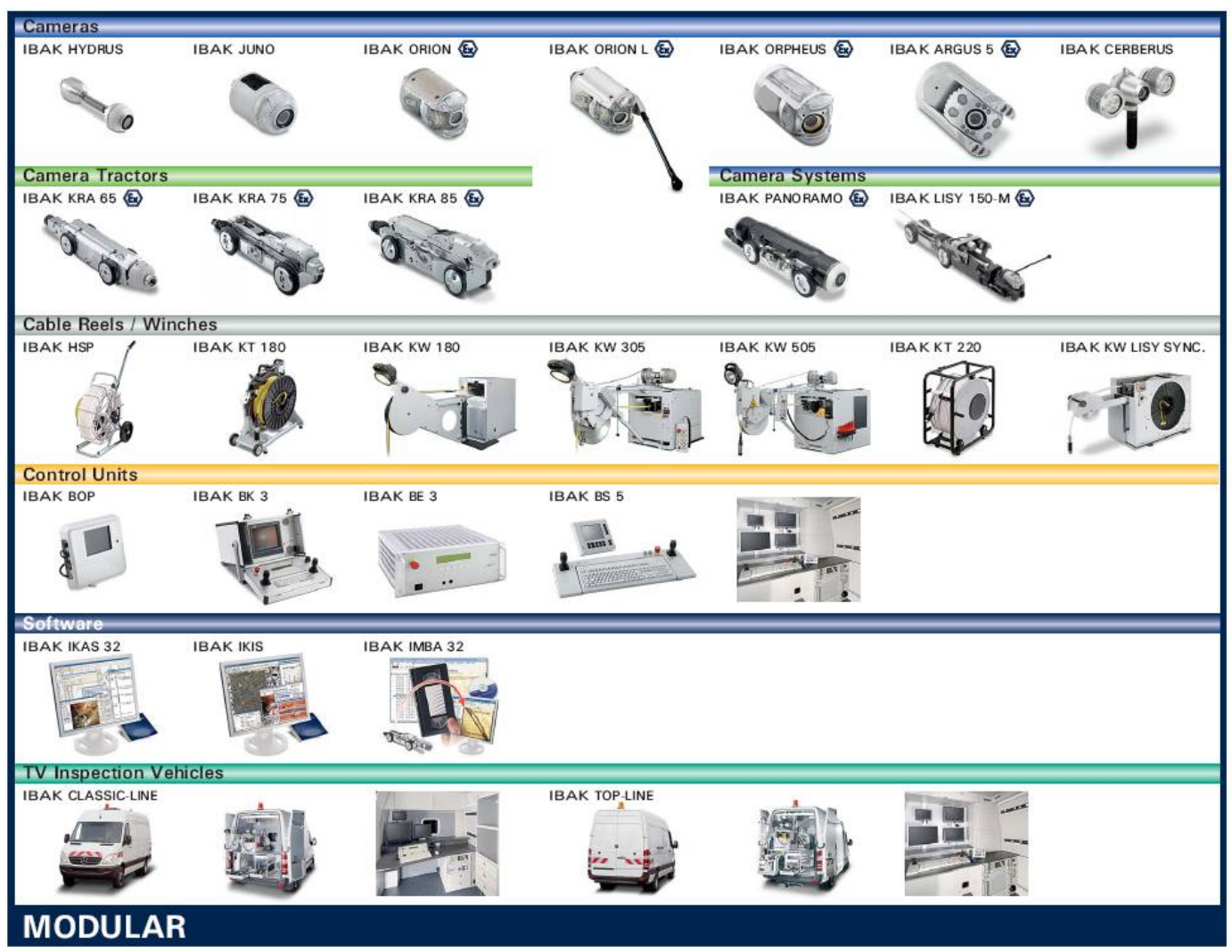

Figure 2.1: IBAK robotic systems involve one of each of these sections

control system required, this is a grouping of component lines four and five of Figure 2.1. The sixth component line, TV Inspection Vehicles is not applicable to the New Zealand pipe inspection industry due to the transport costs related to getting these vehicles here.

\section{Tractors}

The 4 tractor units designed by IBAK are the Argus (Figure 2.2(a)), Triton (Figure 2.2(b)), Sirius (Figure 2.2(c)) and KRA (Figure 2.3) units. The Argus pipe inspection robot is a modifiable system which incorporates a camera and laser distance measurement system. The system uses halogen lighting to illuminate the interior of the pipe and is designed for operation in pipes from $200 \mathrm{~mm}$ in diameter upwards. The robot incorporates a 1/3" colour CCD-sensor camera with 2 x zoom which outputs a PAL or NTSC video to the user. The camera is mounted on an adjustable height arm allowing for the camera to be placed 


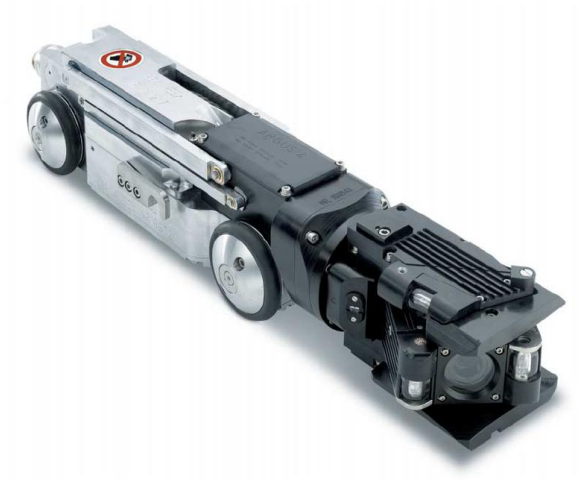

(a)

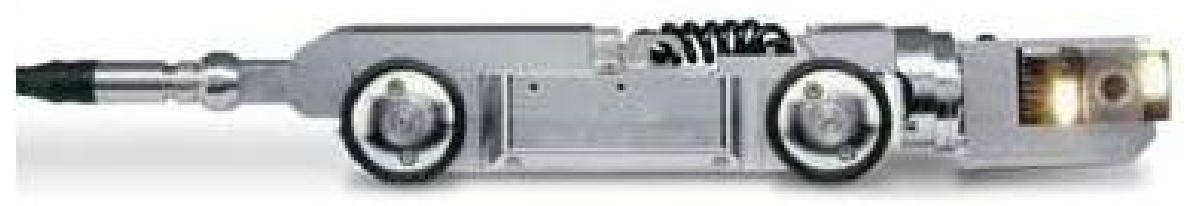

(b)

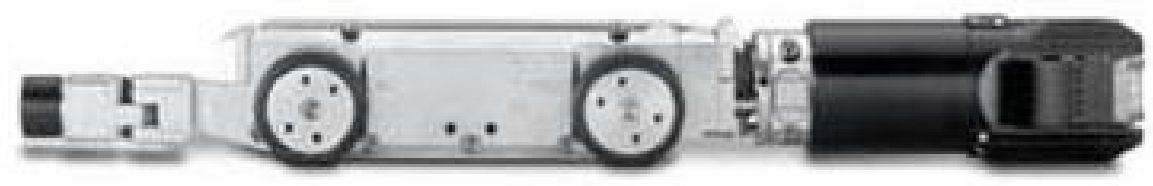

(c)

Figure 2.2: The IBAK (a) Argus, (b) Triton and (c) Sirius tractor units

in the centre of the pipe and has a rotation and pan range of $340^{\circ}$ and $240^{\circ}$ respectively. The robot is $120 \mathrm{~mm}$ wide and $432 \mathrm{~mm}$ long with a maximum adjustable height of $175 \mathrm{~mm}$. It weighs a total of $38 \mathrm{~kg}$ without additional accessories added.

Both the Trition and Sirius units are similar to the Argus system other than that they are for operation in $150 \mathrm{~mm}$ pipes and $100 \mathrm{~mm}$ pipes respectively and these systems do not incorporate the extendable camera arm present in the Argus system.

There are two versions of the KRA system; the KRA 65 system is a robust pipeline inspection chassis for operation in $100 \mathrm{~mm}$ pipes upwards. It has a length to width to height ratio of $345: 79: 68 \mathrm{~mm}$ and weighs $5.5 \mathrm{~kg}$ without camera and lighting additions. Camera and lighting options include the IBAK 


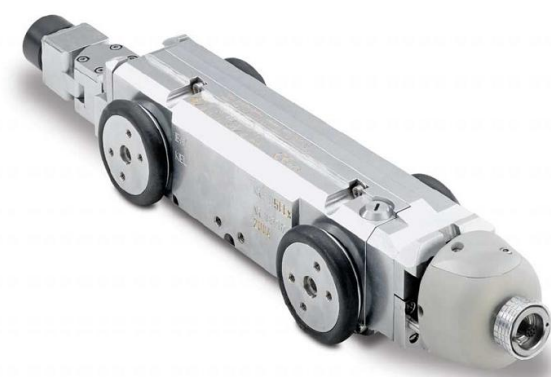

(a)

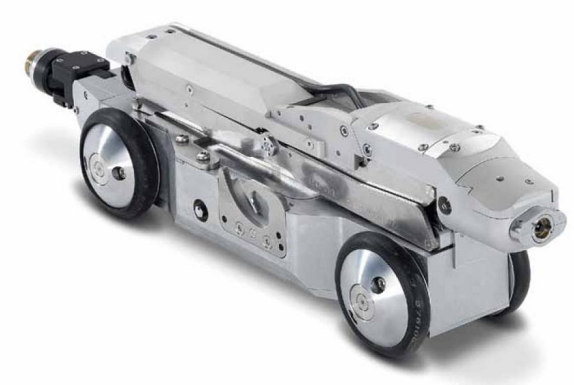

(b)

Figure 2.3: The (a) KRA 65 and (b) KRA 85 robotic systems

Juno, Orion or Orpheus modules which add between $0.5 \mathrm{~kg}$ and $1.6 \mathrm{~kg}$ to the overall weight of the unit.

The larger KRA 85 system is designed for use in $225 \mathrm{~mm}$ pipes upwards. It has a length to width to height ratio of 485:115:68 $\mathrm{mm}$ and weighs $21 \mathrm{~kg}$ without additional components including camera, electric camera lift and pneumatic tyres. With attachments the unit can weigh up to $46 \mathrm{~kg}$. Both these units contain pressure sensors and pressurisation inputs for detection of seal compromise. These robotic units also have the option of attaching three types of cameras.

\section{Cameras}

The IBAK KRA systems have the possibility of being integrated with multiple camera options dependant on the diameter of the pipe being inspected. The smallest options are the IBAK Juno (Figure 2.4(a)) and IBEK Orion (Figure 2.4(b)) camera/lighting modules which will operate in pipes of $100 \mathrm{~mm}$ diameter or greater. The other camera option is the IBAK Orpheus (Figure 2.4(c)) which can operate in pipes greater than $150 \mathrm{~mm}$ in diameter. The Orpheus camera module also has 2 integrated laser diodes for reference measurement. A comparison of the three camera options is shown in Table 2.1. 


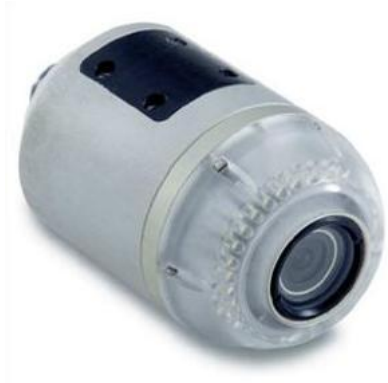

(a)

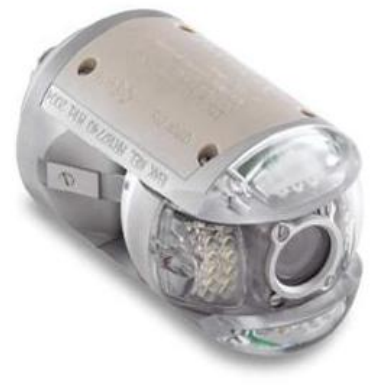

(b)

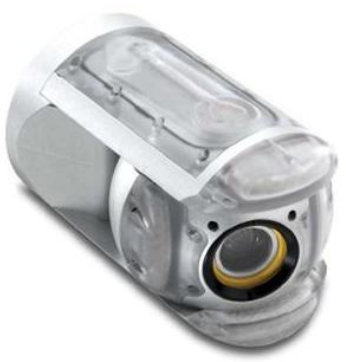

(c)

Figure 2.4: The IBAK (a) Juno, (b) Orion and (c) Orpheus camera module attachments for the KRA tractors

Table 2.1: IBAK Camera Comparison

\begin{tabular}{lccccccc}
\hline \hline Camera & $\begin{array}{c}\text { Diameter: } \\
\text { length }(\mathrm{mm})\end{array}$ & $\begin{array}{c}\text { Weight } \\
(\mathrm{kg})\end{array}$ & $\begin{array}{c}\text { Lighting } \\
(\# \text { LEDs) }\end{array}$ & $\begin{array}{c}\text { Optical } \\
\text { Zoom }\end{array}$ & Focus & Rotation & $\begin{array}{c}\text { Pan } \\
(+/-)\end{array}$ \\
\hline Juno & $60: 100$ & 0.5 & $56^{[1]}$ & None & Auto & None & N/A \\
Orion & $60: 100$ & 0.5 & $40^{[1]}$ & None & Auto & Cont $^{[2]}$ & $120^{\circ}$ \\
Orpheus & $96: 160$ & 1.6 & $88^{[1]}$ & $10 \times$ & Auto & Cont $^{[2]}$ & $120^{\circ}$ \\
\hline
\end{tabular}

${ }^{[1]}$ strength of lighting is adjustable

${ }^{[2]}$ Continuous $360^{\circ}$ rotation is possible

\section{Reels}

IBAK has two types of reels that are applicable for use with the tractor units, motorised truck mounted reels and manual portable units. If a motorised reel is desired, then the selection of reel type is dependant on the maximum size of the unit that is to be used and the size of the vehicle that the reel is to be stored and used within. If a portable system is required, the terrain that the reel must cross is the major factor in deciding which reel to use.

\section{Controllers}

The control systems designed by IBAK to move their tractor units are the BK 3 and BS 5 units shown in Figure 2.1. The BK 3 is a portable control unit with a small TV screen inbuilt, keyboard and dual joysticks for full control of the robotic unit, and can be purchased with the option of adding data acquisition software so that the video received from the robot can be recorded. The BS 5 system is a vehicle mounted system that comes with an ergonomically de- 


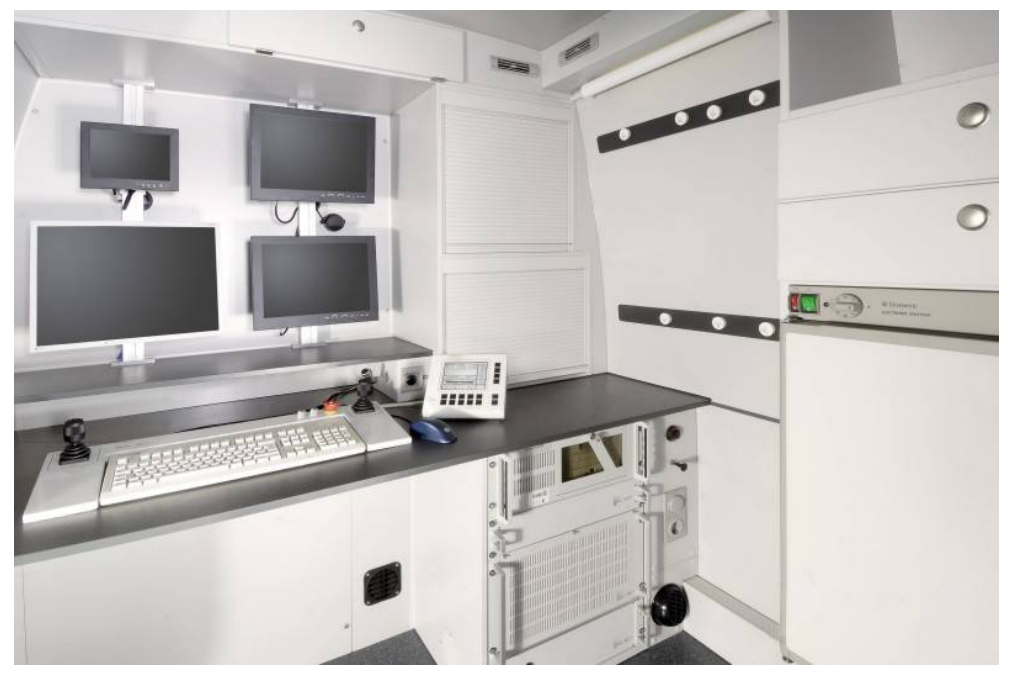

Figure 2.5: BS 5 setup example showing control unit on bench.

signed keyboard and joystick setup for full control of the robot and reel movement; a central control unit is also provided for additional control of the unit (setup example of this system is shown in Figure 2.5). For either of these control systems to record the video from the robot, one of the two IKIS software programs has to be purchased and installed on the control station. IMBA software is then required to convert this to a usable format back at the office.

\subsubsection{Radiodetection PearPoint}

The current systems used by AES are PearPoint systems purchased from Radiodetection $\operatorname{Ltd}[1][5]$.

\section{Tractors}

AES currently use the P400 series of main line tractors. They are designed for use in storm water or sewage pipe and are four or six wheeled tractors that are able to overcome debris encountered in the pipes. The tractors are made from robust stainless steel and anodised aluminium. They are compact in length so that they can be easily inserted into man holes. This series of tractors consists of the P420 unit (Figure 2.6(a) left and middle) which is a six wheeled unit with 


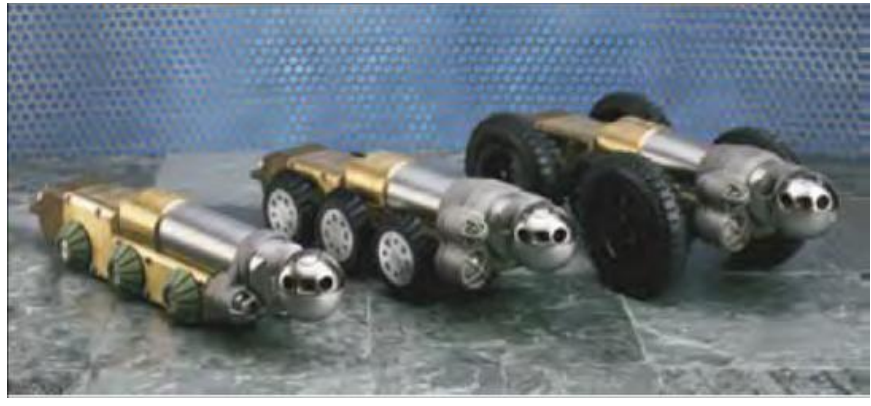

(a)

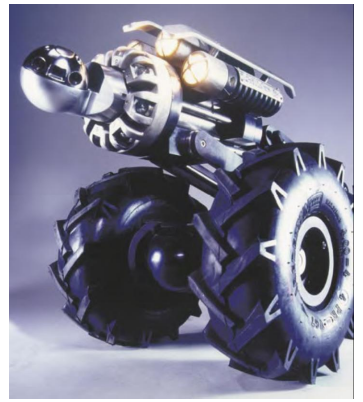

(b)

Figure 2.6: From left to right: (a) P420 with small wheels, P420 with large wheels, P400 and (b) P448

the option of attaching different sized wheels depending on the size of the pipe being inspected. It is able to operate in pipes between $150 \mathrm{~mm}$ and $750 \mathrm{~mm}$ in diameter and can provide up to $110 \mathrm{~W}$ of lighting.

The P400 (Figure 2.6(a) right) tractor is a larger, four wheeled unit with a remote controlled elevator used for insertion and removal into pipes. This unit is used to inspect pipes from $300 \mathrm{~mm}$ upwards with the ability to provide $200 \mathrm{~W}$ of lighting. Both of these tractor units are only able to travel in a straight line. The final P400 series tractor is the P448 (Figure 2.6(b)) which is a large steerable tractor with a remote controlled elevating camera arm and is able to inspect pipes from $500 \mathrm{~mm}$ and upwards. This unit is able to provide $200 \mathrm{~W}$ of lighting. All of the P400 series tractors are able to be mounted with an optional swivel tail cable connector to allow for easier insertion into pipes.

\section{Cameras}

The P494 flexiview $\AA$ Camera from PearPoint is a $360^{\circ}$ continuous pan and tilt camera with better than 1 lux digital video processing. The P494 can be utilised on all P400 series tractors. The standard camera head is designed for inspection of pipes $150 \mathrm{~mm}$ in diameter and larger, whereas the 40:1 auto focus zoom pan and tilt colour camera head option is designed for inspection of pipes $200 \mathrm{~mm}$ and larger. The camera has digital processing and 10× optical zoom followed by $4 \times$ digital zoom camera technology for in-depth inspection of the pipe. The 
P494 camera series comes with directional lighting built into its frame which is rugged and waterproof up to 150 PSI.

\section{Controllers}

The P377 System Control Centre (Figure 2.7) designed for the PearPoint systems is constructed from rugged polyethylene and has a standard laptop "QWERTY" keyboard built into the unit. The unit also contains two bi-axial joysticks and an assortment of function keys that relate to the robot and reel operations. The unit is watertight, displays error messages on the attached screen and is compatible with Composite and SVHS (S-Video) inputs.

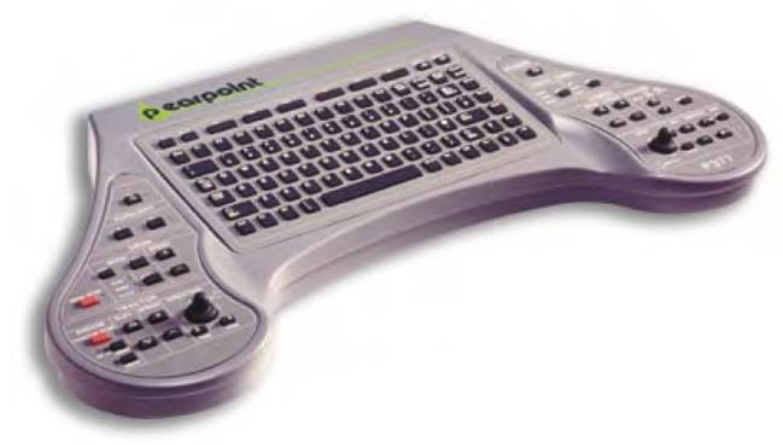

Figure 2.7: PearPoint P377 Control Centre

\section{Portable Robotic Systems}

Radiodetection also sells a range of portable PearPoint inspection systems (Figure 2.8(a)). These systems are able to inspect pipes from $100 \mathrm{~mm}$ to $1200 \mathrm{~mm}$ in diameter, and come with $150 \mathrm{~m}$ of cable on a manual drum which has an inbuilt distance measurement device (displayed in feet). They have a control system with built-in VCR recording and have the ability to attach a camera which can tilt $360^{\circ}$ and pan $270^{\circ}$. The tractors are made from solid brass with stainless steel fittings, not unlike those of the robotic unit being repaired in this project (section 2.2). The portable systems can use one of three possible inspection units, these are the T100, T200 and T300 units (Figure 2.8(b)). 


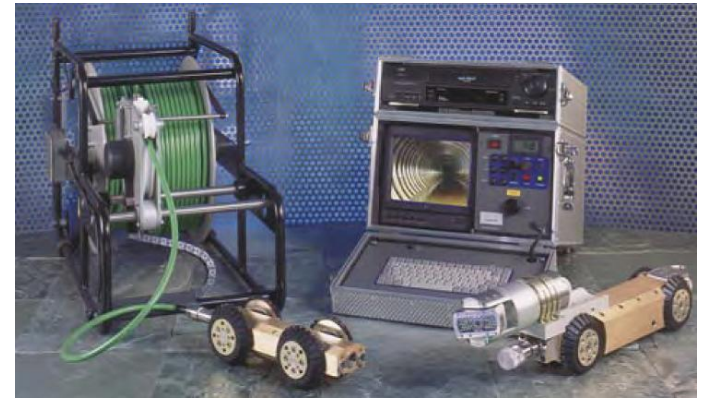

(a)

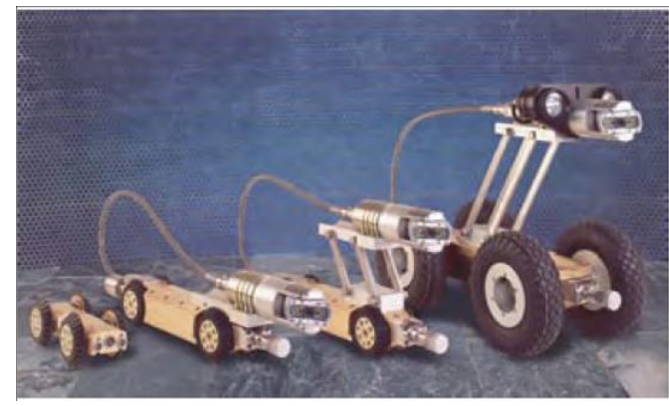

(b)

Figure 2.8: (a) PearPoint portable system and (b) tractor units

The T100 Mini is able to inspect pipes between $100 \mathrm{~mm}$ and $250 \mathrm{~mm}$ in diameter and contains a built-in straight view camera. The T200 Midi unit is a simple modular unit with a pan and tilt camera. An elevated platform can be attached to this robot to allow for inspection of larger pipes from $160 \mathrm{~mm}$ to $750 \mathrm{~mm}$ in diameter. The T300 unit is the largest of the three and is able to inspect pipes up to $1200 \mathrm{~mm}$ in diameter with the ability to attach a remote controlled extension arm allowing for the camera to be raised.

\subsubsection{Rovvers}

Another pipe inspection system currently in operation is the Rovver. Two companies produce these (Figure 2.9), they are iPEK [7] and Environsight [8]. The general specifications of a Rovver system is one able to be modified to work in

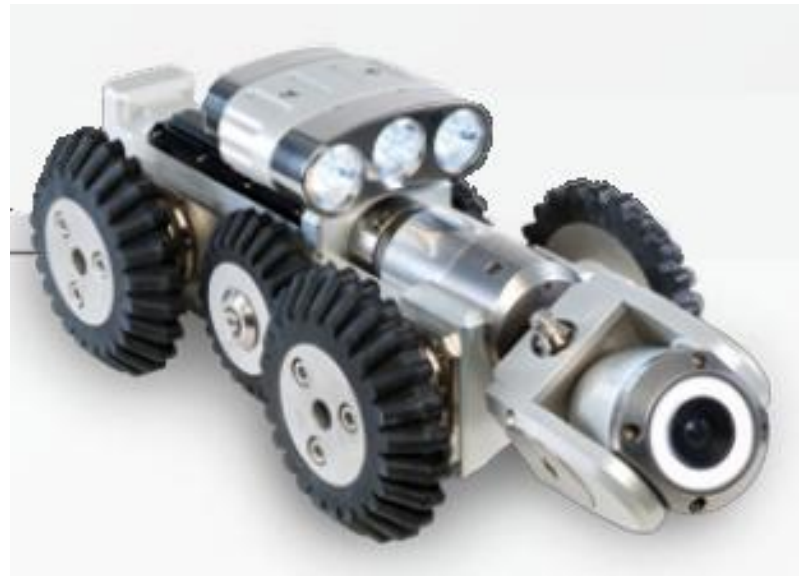

Figure 2.9: Example of Rovver system produced by Envirosight 
pipes ranging from $100 \mathrm{~mm}$ to $1.5 \mathrm{~m}$ in diameter with a camera resolution up to 450 Television Lines (TVL) (equivalent to $600 \times 450$ pixels using the screen aspect ratio of 4:3). The difference in design between the two companies is mainly in their control systems. iPEK systems are designed to be operated within a short distance of the transport vehicle and the Environsight systems include both vehicle mounted and portable systems for all of their Rovver units.

As the robotic system being designed is to be portable, the Enivronsight systems is the most applicable. Their systems are classed as a modular design as all components are interchangeable. This includes being able to attach a different control unit, reel, camera, or tractor to an existing system with minimal effort and no miscommunication (Figure 2.10). This system is able to be controlled via a portable hand pendant or keyboard pendant. These units do not have a video display on them but they can be connected to a portable or vehicle mounted control unit which does. Other options such as a computer or digital video recorder can be attached to a control unit with or without an in-built screen. The reels that Environsight supply are a portable manual reel and a vehicle mounted motorised reel. These reels are interchangeable, allowing them to connect to any of the control stations and tractor units supplied by Environsight. The tractor units supplied are the Rovver 100, 125 and 225 crawlers shown in Figure 2.10. These can have additional attachments added which include different camera modules as well as different wheel sizes and the possibility of an extendable camera arm for adjusting the height of the camera inside the pipe (displayed on Crawler 225 in Figure 2.10). The camera modules are shown on the far right of Figure 2.10 and include straight axial cameras as well as pan/tilt and zoom cameras.

Due to scarcity of information provided on these systems, limited specifications can be found. What can be deduced is that the robotic units start from 


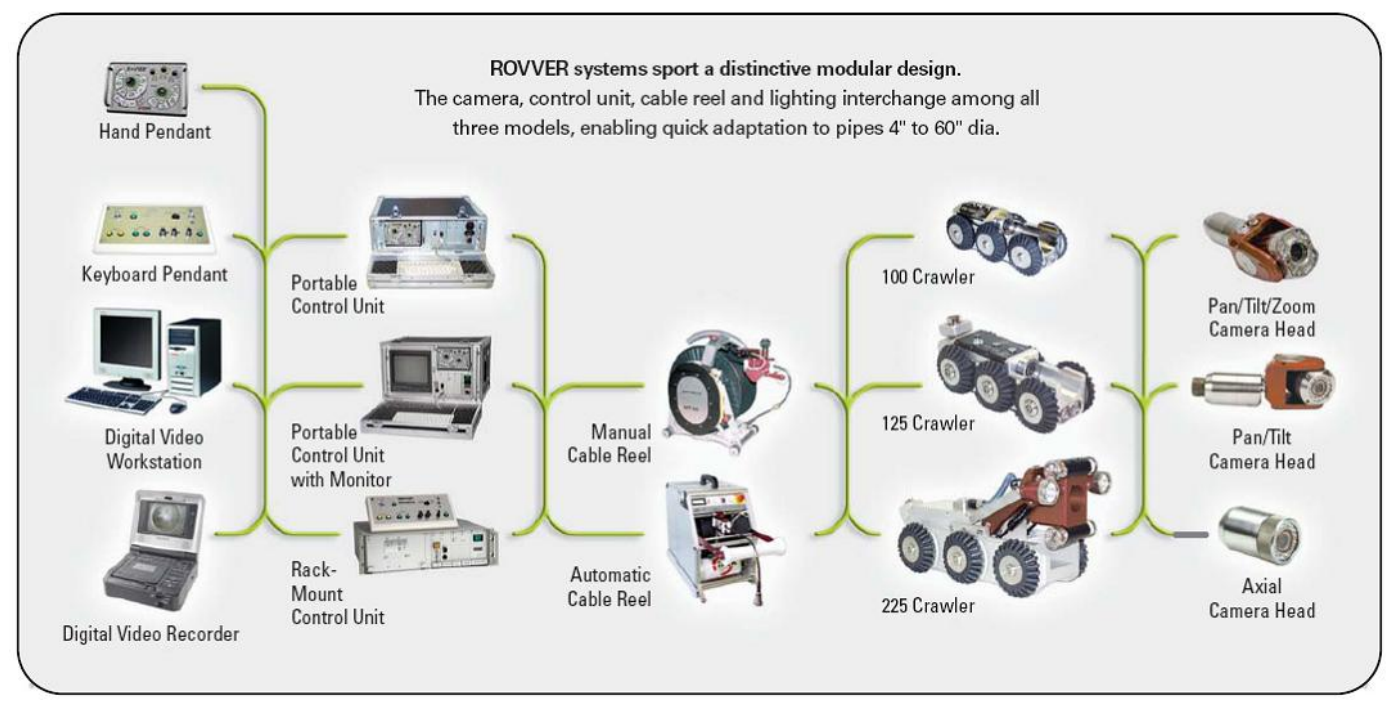

Figure 2.10: Environsight modular system design

under $100 \mathrm{~mm}$ in width and height up to dimensions similar to the IBAK K85 units (section 2.1.1, $115 \mathrm{~mm}$ in width). The cameras in these units are able to provide a resolution of 450 TVL with zoom pan and tilt operations possible. The system itself has a control station able to display video and control the movement of the robot with a joystick.

\subsubsection{Summary}

From all of these systems it is possible to conclude that the diameter of pipes inspected can range from $100 \mathrm{~mm}$ in diameter up to $1.5 \mathrm{~m}$ with different sized robots being required in order to centre the camera in the middle of the pipe. Optional extras that can be incorporated to the robotic units include an extendable camera arm which allows the camera to be raised, centring it in the pipe to give an equal view to all sides as well as preventing damage to the camera caused by debris in the pipe. This is useful although not essential as a good quality camera is able to compensate for non-central positioning and a robust camera housing should prevent damage occurring.

The best camera resolution reported in pipe inspection systems is $450 \mathrm{TVL}$ with a zoom of $120 \times(12 \times$ optical and $10 \times$ digital $)$, although digital zoom is 
Table 2.2: Ideal Robotic System Requirements

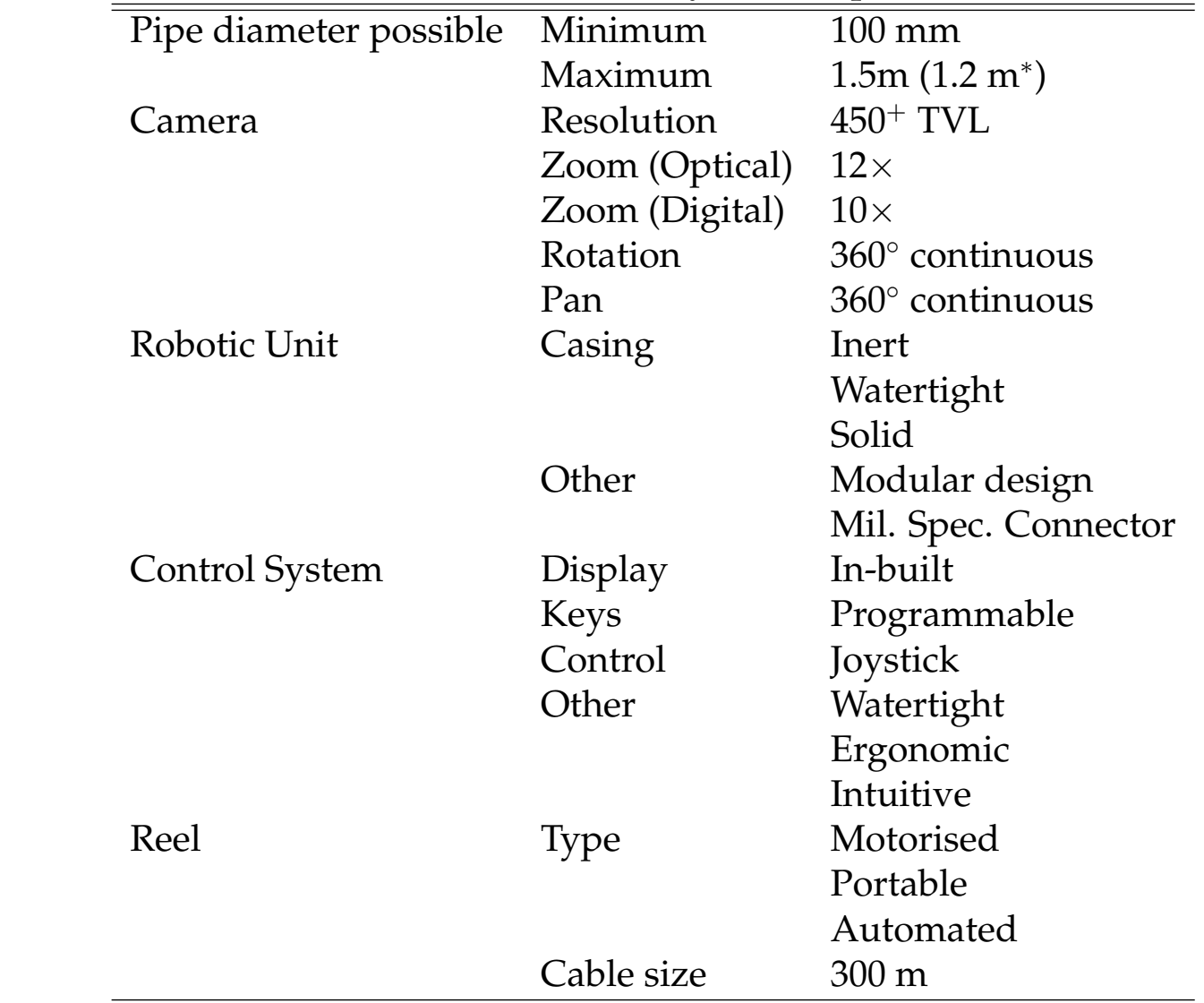

* For a portable system

just a re-sampling of the image and does not truly increase the resolution of the image captured.

Systems that incorporate motorised reels are not portable, and the portable systems with manual reels come with less than $200 \mathrm{~m}$ of cable. These units can be used to inspect pipes between $100 \mathrm{~mm}$ and $1200 \mathrm{~mm}$ in diameter. The control systems are watertight and are fully custom made with built-in joysticks and keys. Some units contain customisable programmable keys while others contain some pre-programmed keys and a QWERTY laptop keyboard. The majority of these control systems do not contain built-in screens, those that do are small low resolution displays.

The maximum distance of inspection of any of these systems is stated at $300 \mathrm{~m}$ with the vehicle mounted reels and control systems. 
All of the cable attachments that are present on the robots researched are military specification connectors which are designed to be rugged whilst providing a strong and secure connection.

Thus the ideal specifications for a portable robotic system would be those displayed in Table 2.2. If most of these specifications are met then the system designed will be able to compete in today's market.

\subsection{The Non-Functional Robotic Unit}

The unit that is to be modified for this project (Figure 2.11) comes with an old camera which can display up to 460 Horizontal TVL and 400 Vertical TVL in PAL format. The minimum pipe diameter that this robotic unit is able to inspect is $150 \mathrm{~mm}$ due to its physical dimensions of $120 \mathrm{~mm}$ in both height and width; the unit is also $630 \mathrm{~mm}$ in length. Additionally this robotic unit weighs $20 \mathrm{~kg}$ due to the main body being constructed of $12 \mathrm{~mm}$ thick solid brass walls.

It is four wheel drive and has the ability to inspect $360^{\circ}$ of the pipe in $460 \mathrm{TVL}$ resolution. It is only able to travel in a straight line but the head of the robot, housing the camera, is able to pan $270^{\circ}$ and rotate $370^{\circ}[9]$ making it a highly useful system. The main body of the robot houses two geared motors, a rotary encoder, two motor drivers, the main control board, a $300 \mathrm{~V}$ to $24 \mathrm{~V}$ DC to

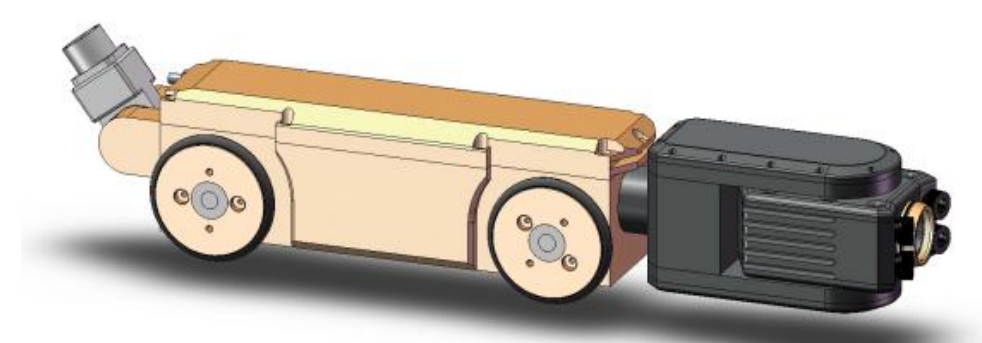

Figure 2.11: SolidWorks model of the Optimess Crawler robot used in this project, (C) author. 


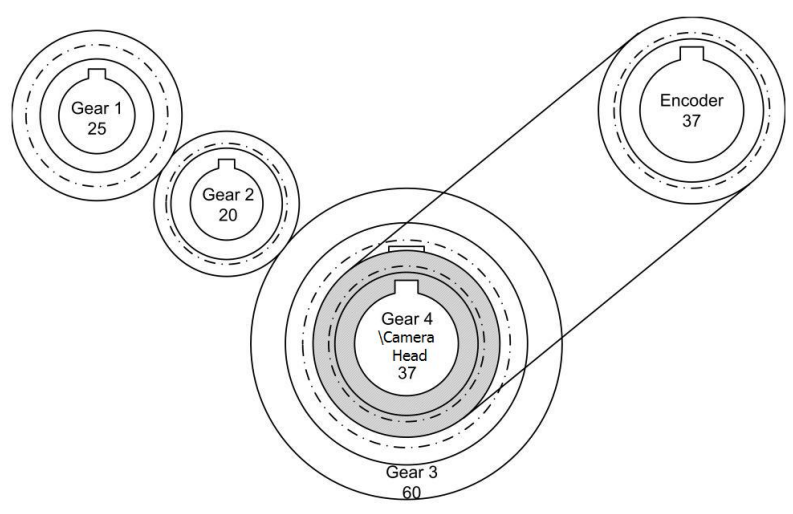

Figure 2.12: Gearing configuration of camera tilt system showing driving gear, translation gear, driven gear on camera head shaft and encoder.

DC converter with external protection circuitry and all necessary wiring. Due to these size constraints, the designing of compact circuitry is an important consideration.

A rotary encoder inside the robot is used to measure how far the camera head has rotated. It is connected to the driven shaft of the camera head by a $1: 1$ belt driven gearing system shown as Gear 4 in Figure 2.12. This diagram also shows that the camera shaft is driven by another gearing system. This system attaches to a $12 \mathrm{~V}$ (all voltages are direct current unless otherwise stated) motor at gear 1, with gear 2 acting as a scaling gear such that the ratio between gear 1 and the camera shaft gear (gear 3 ) is 25:60, or 5:12. Thus the camera speed of rotation is $\frac{5}{12}$ that of gear 1 and the torque produced is $\frac{12}{5}$ greater. The $12 \mathrm{~V}$ motor is connected to gear 1 via a planetary gear box which translates the torque produced by the motor to overcome the weight of the camera head (the motor and gearbox are defined in section 6.2.5).

In this head an analogue Sony camera (section 6.2.1) is housed along with four high powered LEDs (section 6.1) for illuminating the inside of the pipe, a motor to control the panning of the camera and a camera control board (section 4.1). The camera control board handles the transmission of control signals to the camera (such as focus and zoom), the transmission of the video signal back 


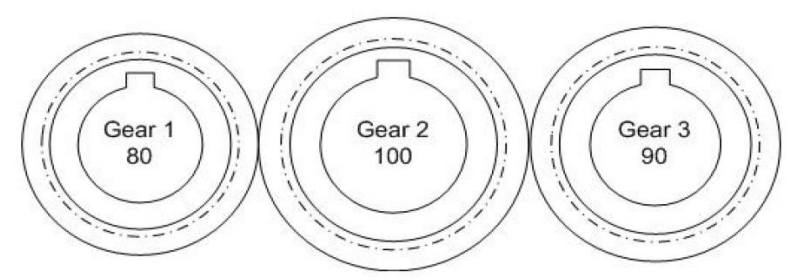

Figure 2.13: Camera pan gearing diagram.

through the robot to the user, and provides the power to the LEDs and camera. The internal section of the head is only able to fit a control board within a $57 \mathrm{~mm} \times 57 \mathrm{~mm}$ space, hence the circuit must be compact. The panning of the camera is accomplished with the gearing system shown in Figure 2.13. This minimal torque increase of $\frac{9}{8}$ allows the controlling motor to be housed at the back of the camera head whilst the pivot point is forward of centre (shown in Figure 2.14). To overcome the weight of the camera head and to allow for smoother panning, a planetary gearbox is inserted between the motor and this gearing system.

The drive motor of the robot is a $24 \mathrm{~V}$ motor that also has a planetary gearbox attached to it. This is a $66: 1$ gearbox that is connected to the wheel axle via a bevel gearing system (Figure 2.15(a)) as the direction of rotation needs to be switched $90^{\circ}$ to allow the motor which is housed long ways in the robot to drive the wheels which are perpendicular to it. This bevel gearing system is a further 2:1 translation that gives the robot a slower overall speed whilst increasing its torque throughput to 132 times that of the motor. The larger of

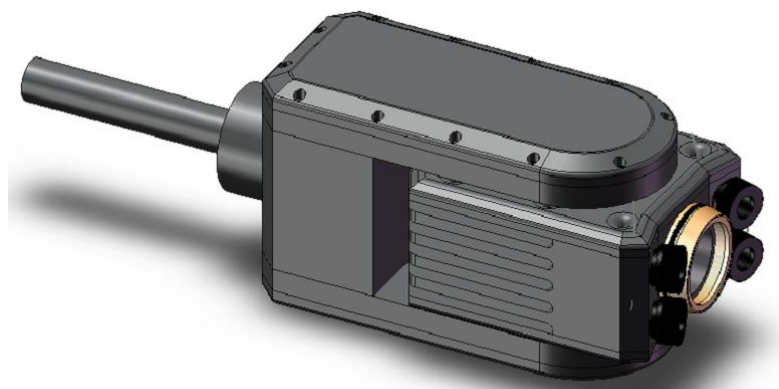

Figure 2.14: SolidWorks model of camera head, (c) author. 


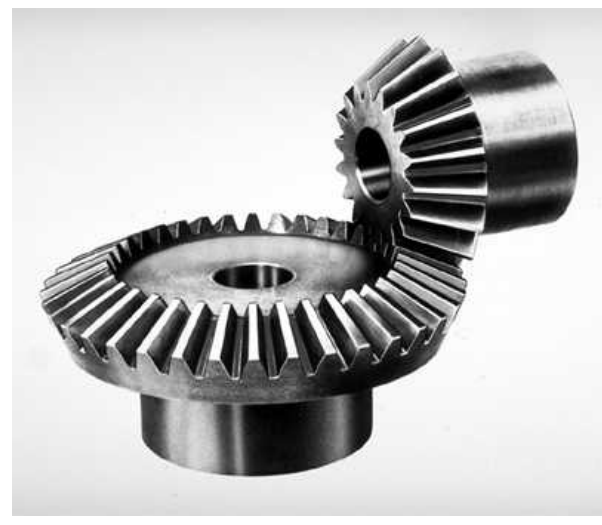

(a)

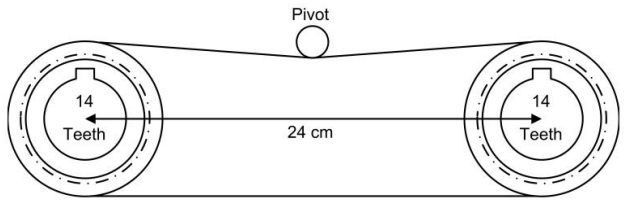

(b)

Figure 2.15: (a) straight toothed bevel gear system [10] and (b) front and rear axle chain connection diagram, both of which used in driving of robot.

the two bevel gears is directly connected to the rear wheel axle of the robot. To make this robot four wheel drive, an additional gearing system has been employed in the form of a chain driven system connecting the front and rear axles with a 1:1 ratio (Figure 2.15(b)).

\subsection{Previous Work}

This project was started by another Victoria University of Wellington Masters student, Aleks Ristic, in 2008[11]. During his work Ristic was able to reverse engineer or redesign most of the pipe inspection robot's internal circuitry and create a Graphical User Interface (GUI) in LabVIEW (Figure 2.16). The internal circuitry of the robot involves a main control board which is designed to implement serial communication between the computer and the robot, a camera control board to control the camera and the four lighting LEDs and three motors. Two of the motors control the pan and tilt of the camera head with the third controlling the forward movement of the robot. The final part of the internal circuitry is the Vicor power module which converts the incoming $300 \mathrm{~V}$ to $12 \mathrm{~V}$ (at $10 \mathrm{~A}$ maximum output) and the power module protection board. 
year. During this time AES field technicians found the LabVIEW GUI to be extremely hard to use and requested for it to be changed. Ristic had started the conversion into Visual Basic with the .NET framework setting up the initial serial communication and adding skeleton code required for the activation and controlling of the robot. The methods were setup in such a way that the robot was controlled by clicking on the appropriate command button in the GUI.

By the end of his tenure at AES Ristic was able to control the robot via the LabVIEW code, with the video from the camera displayed on the computer screen, but had not yet implemented the Visual Basic GUI. Control is only possible when the robot was directly connected to the computer, and not via the cable.

Hence the broad objective of this project is to expand on the work done by Ristic such that a robust and reliable system is able to be designed with today's standards in mind.

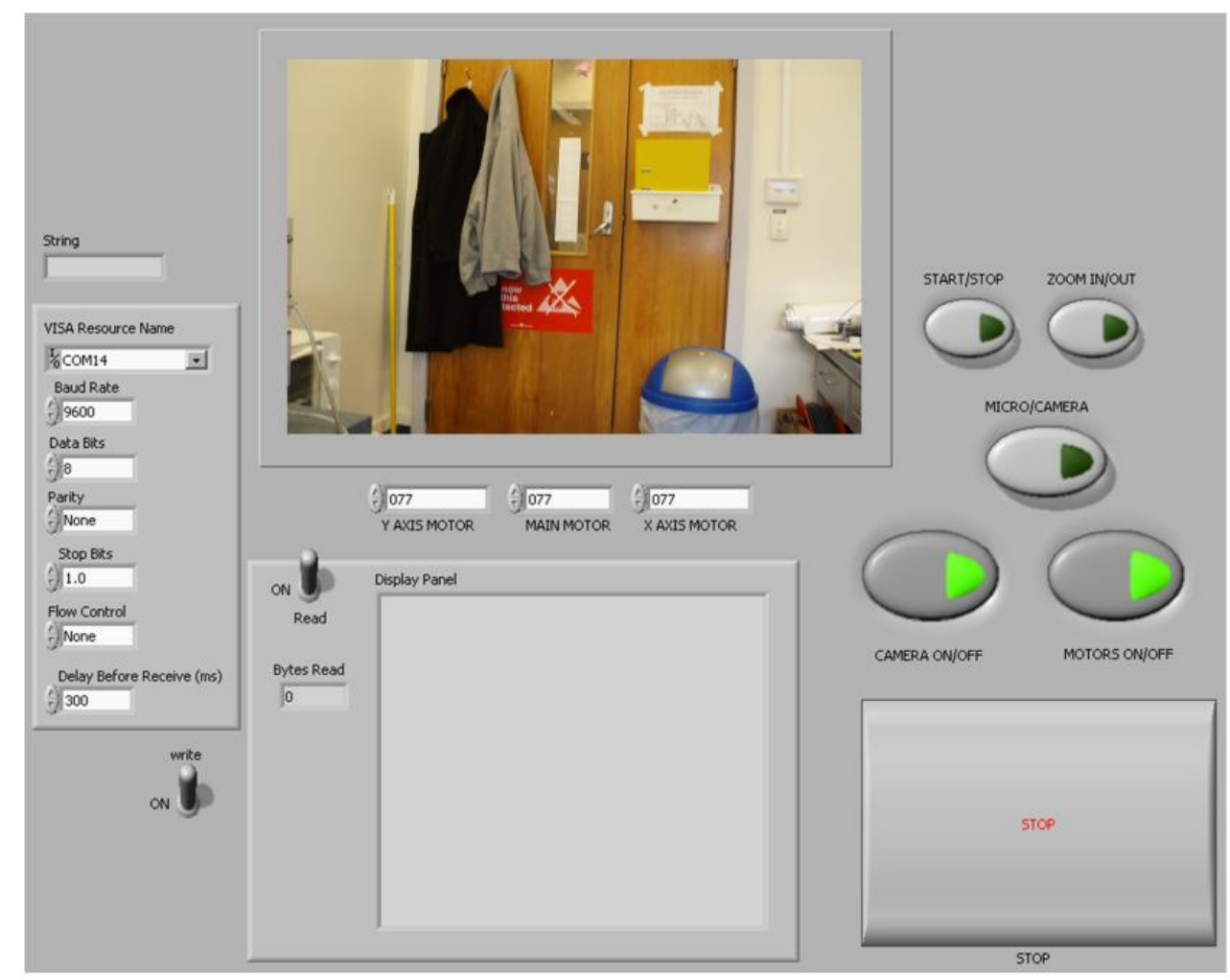

Figure 2.16: Mr Ristic's LabVIEW GUI 



\section{Chapter 3}

\section{Communication and Transmission}

\subsection{Overview}

This project requires several sub-systems to communicate with each other. These sub-systems are the control system which the user interacts with, the base station which is designed to interpret information from the computer, the robotic unit and the camera inside the robot. This section outlines communication protocols that were considered for use in this project.

Due to the communication distance required in this project, parallel data transmission is not possible hence serial asynchronous data packet transmission is utilised.

Data packet transmission allows for a large amount of data to be sent to a device. This requires encoding characters into the standard serial pulse stream. A common format used for this encoding is ASCII (American Standard Code for Information Interchange). ASCII contains 128 characters, 95 of which are standard printable characters including the space character. 


\subsubsection{RS-232}

Recommended Standard 232 (RS-232) is one of the most common forms of serial data communication in use. It is a set of standards that specify the electrical, functional and mechanical configuration required to interface to devices running this standard. The advantages of this style of communication are its ability to run in both synchronous and asynchronous data transmission modes and the ability to send and receive data at the same time. The setup of this communication requires that the transmission line of device 1 is connected to the receive line of device 2 and vice versa. The major disadvantage of RS-232 communication is the maximum distance that a signal can travel is approximately $15 \mathrm{~m}$ without the use of costly low capacitance cables. These cables can expand the maximum distance to $300 \mathrm{~m}$ but this does not allow the data to be transmitted at the maximum speed of $20 \mathrm{~kb} / \mathrm{s}[12][13]$.

RS-232 is used in this project as the programming of the microcontroller requires a serial interface to the computer. RS-232 is easy to implement and requires low cost components. An external RS-232 to USB converter is required for use with modern computers as serial ports are no longer standard.

\subsubsection{RS-485}

Recommended Standard 485 (RS-485) is also known as EIA-485 after the Electronic Industries Alliance. It defines a standard that uses a tri-state driver and is most commonly used for point-to-point long range data transmission. RS485 communication involves the use of a differential twisted pair cabling system which allows the signal to travel longer distances than RS-232. Taking the data signal from the measured difference on the two transmission lines gives the RS-485 standard its long range advantage as well as its singular direction disadvantage. This communication standard is not able to simulta- 
neously transmit and receive signals unlike the RS-232 standard. RS-485 can however run significantly faster than RS-232 with a theoretical maximum data rate of $35 \mathrm{Mb} / \mathrm{s}$ over $12 \mathrm{~m}$. Due to losses in cabling $10 \mathrm{Mb} / \mathrm{s}$ is usually specified as the usable maximum[14]-[16]. RS-485 communication can also run a "Master/Slave" communication arrangement.

The Master and Slave arrangement is where one device is programmed to be the Master and controls another device known as the Slave. In RS-485 communication this Master is able to control up to 32 Slaves on the same communication line by giving each Slave a unique identifying name. In naming each Slave, the Master is able to transmit data specific to one Slave without other devices misinterpreting the data. The Slave name is the first character sent down the transmission line which notifies the device if the data is for it or for another device. If the system is configured so that the Master sends an instruction and waits for a response before transmitting again then the Slave is also able to transmit on the line. In this configuration both the Master and Slave need to be reverted back to a receiving state after transmission otherwise the signal will not be read. Thus the coding for a Master Slave arrangement is more complex than for a RS-232 communication setup[12].

\subsubsection{USB}

Universal Serial Bus (USB) communication uses a master-slave configuration known as a host-client interface, in this the host is usually a computer. USB devices are the most common peripheral device connection in personal computers with all currently manufactured desktop and laptop computers containing a USB interface. Due to this, the option of using USB for controlling of the base station is to be considered[17].

In 2000 USB 2.0 was introduced which took the maximum speed of USB devices from $12 \mathrm{Mb} / \mathrm{s}$ to $480 \mathrm{Mb} / \mathrm{s}$ with a $5 \mathrm{~V} 500 \mathrm{~mA}$ power supply, although 
the sustained read and write data rate of USB is $264 \mathrm{Mb} / \mathrm{s}$ and $216 \mathrm{Mb} / \mathrm{s}$ respectively. The maximum distance that a USB cable can be is $5 \mathrm{~m}$ although a maximum of 5 USB hubs can be connected to a single line expanding the distance to $30 \mathrm{~m}[18]$.

Using USB as a computer to board interface requires more costly on-board components than the RS-232 configuration that it would replace, but would allow for a more user-friendly setup as an external RS-232 to USB converter would not be required.

\subsubsection{Firewire}

FireWire is an Apple corporation invention known as the IEEE-1394 standard after the Institute of Electrical and Electronics Engineers proposed to replace the Small Computer System Interface (SCSI) with it. It is a peer-to-peer network where each node, or device is considered equal. FireWire is able to carry data up to $4.5 \mathrm{~m}$ at $400 \mathrm{Mb} / \mathrm{s}$ but with a sustained read and write data rate of $304 \mathrm{Mb} / \mathrm{s}$ and $280 \mathrm{Mb} / \mathrm{s}$ which is 40 and $64 \mathrm{Mb} / \mathrm{s}$ faster than USB 2.0 respectively [17]. Due to FireWire not being standard on laptop personal computers it will not be used in this project.

\subsubsection{Fibre Optics}

Fibre optic communication is a high speed, low loss means of communication. Fibre optic cables are traditionally constructed using a glass core, derived from silica, but can also be made from plastic. A plastic core is not suitable for long-distance transmission as it experiences large transmission losses compared to quartz cores[19]. The core is protected, insulated and strengthened by adding layers of plastic, metal and insulation materials. The glass cores have the advantages of extremely small transmission losses over long distances and 
large bandwidth. The bandwidth is dependent on the distance the data has to travel, an example of which is a common multi-mode fibre cable which has an approximate bandwidth of $500 \mathrm{MHz} / \mathrm{km}$, resulting in $1666 \mathrm{MHz}$ over $0.3 \mathrm{~km}$ (the maximum length of cable considered in this project).

Other advantages of fibre optics are their immunity to electromagnetic radiation and interference, they are secure as they cannot be influenced by external sources and they cannot be read by receivers that the cable does not terminate at. The reverse situation is also true, fibre optic cables do not transmit noise to external components making it possible to pack optical cables close together without risk of cross-talk. In multimode communication cables, the internal cores are typically between $50 \mu \mathrm{m}$ and $200 \mu \mathrm{m}$ in diameter.

Two significant disadvantages exist for fibre optics, the first being their cost. Fibre optics were originally developed in 1970 for research purposes using GaAs (Gallium Arsenide) semiconducting lasers. In the late 1990s commercial fibre optic cables were produced to overcome the internet broadband shortages in the United States. Due to this late development of fibre optics for commercial application and the complex manufacturing process required they still cost in the order of USD $\$ 800$ for a $500 \mathrm{~m}$ cable [19]. The second disadvantage is that fibre optics are unable to carry large amounts of power, in fact they are unable to carry enough power to supply any form of electrical device and so the robot would need to be powered by some means other than via an optical tether cable[12][20].

\subsubsection{Communication Summary}

RS-232, RS-485 and USB protocols will be used in this project. Integrating this into a singular system will allow the computer to control the reel and robot as well as be able to read the signals returned by the reel, robot and camera. 


\subsection{Video Setup and Standards}

Interfacing to a video source requires knowledge of the video standard that the device is running on. This is true for both a television and a camera as the standard on which they run defines the boundaries of operation for these devices. Many systems can handle multiple standards; hence the selection of the standard to use may not be defined by the hardware but instead be defined by the software configuration as selected by the user. Of these video standards there consist two broad spectrums, analogue video and digital video, each of which can use one or more of the video configurations described in section 3.2.4.

Screen flickering is a major problem than can occur in both analogue and digital video display; it is caused by an insufficient frame rate or interlaced scanning. The frame rate of the video defines the number of still images that will be displayed in one second. Too few frames and the image will flicker, too many can overload the processing unit causing the video to lag. Interlaced scanning was developed for CRT (Cathode Ray Tube) televisions to allow for improved apparent picture quality without increasing the required bandwidth or speed of the components. This is done by displaying every second horizontal line of data per scan across and down the screen. Because of this, interlaced video has the problem of twittering if sharp horizontal lines are displayed as every second scan the line would disappear causing the image to flicker. The solution to this is progressive scanning which avoids this problem by displaying each line progressively. The problem with this method is that to be able to refresh the screen at the desired frame rate the components are required to run at twice the speed as interlaced systems. 


\subsubsection{Analogue Video}

There are 3 major analogue video standards currently being employed throughout the world; National Television Standards Committee (NTSC), Phase Alternating Line (PAL) and Séquential Couleur Avec Mémoire (SECAM).

NTSC is an analogue video standard used in the United States since 1953. NTSC has significant problems with the display of colours as the reproduction of these colours is determined by the phase of the signal and is severely affected by electromagnetic signal propagation and noise.

PAL was devised in West Germany to overcome the problems inherent in NTSC, and was implemented there, The Netherlands and the United Kingdom in 1967. PAL was designed to invert the colour signal by $180^{\circ}$ on alternative lines allowing this standard to reproduce the video colours as they were originally intended. This standard is used in the majority of Western Europe, Africa and Oceania, including New Zealand.

SECAM is the television standard in France derived from PAL. Its translates to "sequential colour with memory". The name originates from two colour signals being sent sequentially, hence the first part needs to be stored while waiting for the second part before the image can be displayed. As well as being used in France it is also used in parts of the Middle East which have French influence[21].

\subsubsection{Digital Video}

Digital video offers the advantages of enhanced bandwidth efficiency through compression, improved signal quality and more effective management and control of the signal. Under the category of digital video comes the well known acronym HD, or High Definition. This is a form of digital video that has been 
defined as a video signal that has a minimum resolution of $720 \times 1280$ at 24 , 30, or 60 frames per second by the Advanced Television Systems Committee (ATSC). All other digital video that has a resolution lower than this is classed as Standard Definition (SD). This is summarised in Table 3.1.

Table 3.1: ATSC Digital Video Standards[21]

\begin{tabular}{ccccc}
\hline \hline Standard & $\begin{array}{c}\text { Vertical } \\
\text { Lines }\end{array}$ & $\begin{array}{c}\text { Horizontal } \\
\text { Lines }\end{array}$ & Aspect Ratio & $\begin{array}{c}\text { Frames per } \\
\text { Second }\end{array}$ \\
\hline HD & 1080 & 1920 & $16: 9$ & $24 \mathrm{p}, 30 \mathrm{p}, 60 \mathrm{i}$ \\
& 720 & 1280 & $16: 9$ & $24 \mathrm{p}, 30 \mathrm{p} 60 \mathrm{p}$ \\
SD & 480 & 704 & $16: 9,4: 3$ & $24 \mathrm{p}, 30 \mathrm{p}, 60 \mathrm{p}$ and 60i \\
& 480 & 640 & $4: 3$ & $24 \mathrm{p}, 30 \mathrm{p}, 60 \mathrm{p}$ and 60i \\
$i=$ interlaced, $p=$ progressive. \\
Note: HD is defined as $1440 \times 1080$ in $4: 3$ aspect ratio
\end{tabular}

\subsubsection{Selected video comparison}

Table 3.2 gives a comparison between the three major video standards that are considered in this project. Of these HDTV is the only digital video standard, NTSC is most commonly used in North America and PAL is used locally. The frame rate of the picture is specified in frames per second, either interlaced or progressive video format, and the resolution of the video is specified in pixels per line.

This data provides the basis for making a decision on which video standard to use with a specific hardware configuration, or which video standard to select if more than one option is available.

Table 3.2: Analogue and Digital Video Standards[21]

\begin{tabular}{llll}
\hline \hline Standard & NTSC & PAL & HDTV \\
\hline Analogue/digital & Analogue & Analogue & Digital \\
Horizontal scanlines & 525 & 625 & $640,704,1280$, or 1920 \\
Synchronisation & 40 & 49 & N/A \\
Resolution, ppl & 640 & 640 & 480,720, or 1080 \\
Frame rate, fps & $30 \mathrm{i}$ & $25 \mathrm{i}$ & $24 \mathrm{p}, 30 \mathrm{p}, 60 \mathrm{p}$, or $60 \mathrm{i}$ \\
Aspect ratio & $4: 3$ & $4: 3$ & $4: 3$ or $16: 9$ \\
$i=$ interlaced, $p=$ progressive. & &
\end{tabular}




\subsubsection{Video Configurations}

Peripheral devices exist with multiple possible video connection configurations, the three main configurations are component video, composite video and separate video. Deciding which to use requires understanding of each, including their advantages and limitations. These video configurations transmit the data in the form of a colour space, of which YUV and YPbPr are analogue colour spaces and $\mathrm{YCbCr}$ is a digital colour space. RGB (red, green and blue) is defined as both an analogue and digital colour space depending on the manufacturer.

\section{Composite Video}

Composite video is one of the most common video configurations available today. This configuration normally utilises a yellow RCA (or phono) connector grouped with a white and a red RCA connector for left and right audio. This format uses a "Colour, Video, Blank and Sync" (CVBS) scheme to transmit YUV data with synchronous pluses. The acronym YUV stands for the luminance $(\mathrm{Y})$ and Chrominance (UV) data being sent down the cable, where the chrominance can be split into hue and saturation levels. This video format is the lowest resolution format but due to its commonality it must be considered.

\section{Component Video}

Component video is a video signal that has been split into two or more components. Standard component video configurations consist of three video signals, these signals are either in the RGB, YCbCr or YPbPr colour space. Technically YCbCr and YPbPr are not true colour spaces but rather a colour space derived from RGB using encoding algorithms (see section 3.2.4).

Component video is the only configuration that allows the transmission of dig- 
ital video signals whilst still being able to transmit analogue signals using standard RCA cables.

\section{Separate Video}

Separate Video is more commonly know as S-Video and is considered as a low resolution component video configuration. Separate video is a dual signal carrying setup with 4 or 7 pins, where a 4 pin configuration consists of an intensity (luminance) and colour (chrominance) connection, each with its own ground. A 7 pin configuration contains both the S-Video setup as well as a 2 pin composite video connection, the final pin is not connected. As S-Video contains the intensity and colour data as separate streams it is theoretically better than composite video as composite video combines the two. This is true if and only if the encoding and decoding devices are compatible, otherwise the colours are not displayed correctly.

\section{Colour Spaces}

Of the three spaces used most commonly in video signal display RGB is the one that is most commonly understood. Its derivatives, $\mathrm{YPbPr}$ and $\mathrm{YCbCr}$, are mostly unknown other than by those with in-depth knowledge of video reproduction. Others may recognise the abbreviation of YUV as being the same as $\mathrm{YCbCr}$ or $\mathrm{YPbPr}[24]$. Although the colour spaces are similar this distinction is incorrect. YUV is a well defined and documented colour space whereas YCbCr and YPbPr are conversions of RGB colour space to digital (YCbCr) and analogue (YPbPr) luma and chroma representations of this image. For the con-

Table 3.3: Coefficients of colour conversion from RGB to YPbPr [22][23]

\begin{tabular}{lccc}
\hline \hline Reference standard & $K_{r y}$ & $K_{b y}$ & $K_{g y}$ \\
\hline ITU601 / ITU-T 709 1250/50/2:1 & 0.299 & 0.114 & 0.587 \\
ITU709 / ITU-T 709 1250/60/2:1 & 0.2126 & 0.0722 & 0.7152 \\
SMPTE 240M (1999) & 0.212 & 0.087 & 0.701 \\
\hline
\end{tabular}


version of RGB to YPbPr formulae 3.1 to 3.3 are used where the variables $K_{r y}$ $K_{b y}$ and $K_{g y}$ are substituted with the value specified by the video standard being used. Three sets of standards are displayed in Table 3.3 all of which are digital standards derived from using an 8-bit Digital to Analogue Converter (DAC).

$$
\begin{aligned}
& Y=K_{r y} \cdot R+K_{g y} \cdot G+K_{b y} \cdot B \\
& P b=K_{r u} \cdot R+K_{g u} \cdot G+K_{b u} \cdot B \\
& P r=K_{r v} \cdot R+K_{g v} \cdot G+K_{b v} \cdot B
\end{aligned}
$$

where

$$
\begin{aligned}
0 \leq & \leq 1 \\
-1 \leq & P b \\
-1 \leq & \operatorname{Pr} \leq 1 \\
K_{r u} & =-K_{r y} \\
K_{g u} & =-K_{g y} \\
K_{b u} & =1-K_{b y} \\
K_{r v} & =1-K_{r y} \\
K_{g v} & =-K_{g y} \\
K_{b v} & =-K_{b y}
\end{aligned}
$$

The equations 3.1 to 3.3 are scalable functions that currently range from 0 to 1 or -1 to 1 with no offset. For a digital signal such as the YCBCr configuration, the equations must be offset so that they can vary between the maximum and minimum encoder values. Thus the digital equations become equations 3.4 to 3.6 where the range required is that of the DAC implemented in the device and the offset value is determined by that range and the standard being implemented. As these equations deal in digital quantities, the values of $R$, $\mathrm{G}$ and $\mathrm{B}$ must also be converted to a digital colour space with maximum and minimum values equivalent to those of the DAC. Hence an ADC (analogue to digital converter) with the same range as the DAC must be used in the creation 
of the digital RGB colour space. The digital R, G and B values are denoted by the derivative symbol in equations 3.4 to 3.6 so that it is possible to distinguish them from their analogue parents.

$$
\begin{gathered}
Y=K_{r y} \cdot R^{\prime}+K_{g y} \cdot G^{\prime}+K_{b y} \cdot B^{\prime}+\text { Offset } \\
C b=K_{r u} \cdot R^{\prime}+K_{g u} \cdot G^{\prime}+K_{b u} \cdot B^{\prime}+\text { range } / 2 \\
C r=K_{r v} \cdot R^{\prime}+K_{g v} \cdot G^{\prime}+K_{b v} \cdot B^{\prime}+\text { range } / 2
\end{gathered}
$$

\subsubsection{Video configuration summary}

The most suitable configuration for this application is the component video as it is able to handle high definition video. The colour space used is only significant if a problem in the image colour occurs, in which case the knowledge of colour spaces will allow the problem to be fixed using hardware adaptors. Composite video is a possibility due to its commonality in both cameras and recording devices but is not preferred due to its low resolution. 


\section{Chapter 4}

\section{Robotic Printed Circuit Boards}

Four printed circuit boards (PCBs) were constructed for the robotic unit in this project. This section introduces these boards, including the design considerations and the overall objective of each board as part of an integrated robotic pipe inspection system.

Designing a system that is easy to repair is one of the key requirements specified by Bryan Pobar of AES. How this is implemented is also outlined in this section.

\subsection{Camera Board}

The camera board is designed to control the camera module (section 6.2.1) in the robot's head (hence the shape in Figure 4.1(a)) as well as the four LEDs (section 6.1) that are used to illuminate the inside of the pipe being inspected. The camera board takes a $12 \mathrm{~V}$ and control input from the main control board and a serial communication input from the base station. It returns an analogue video signal to the base station as well as two comparator test values back to the main control board. Hence the camera board has 5 unique in- 


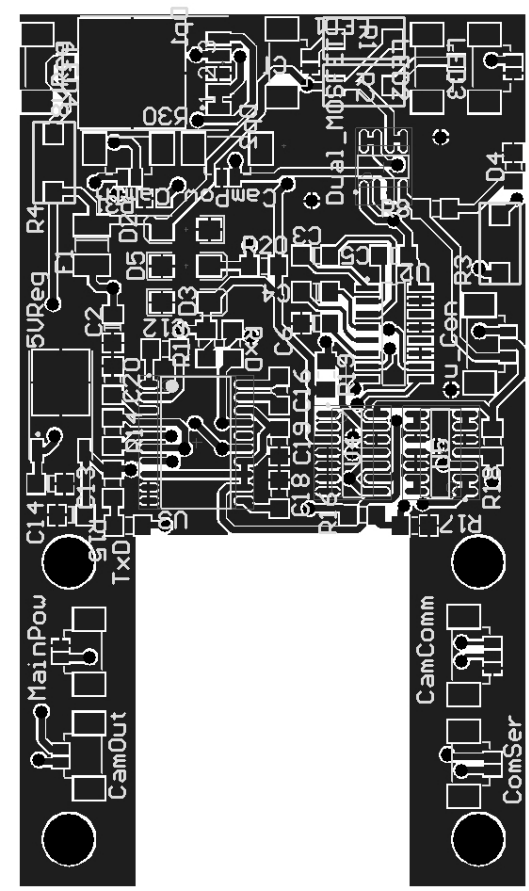

(a)

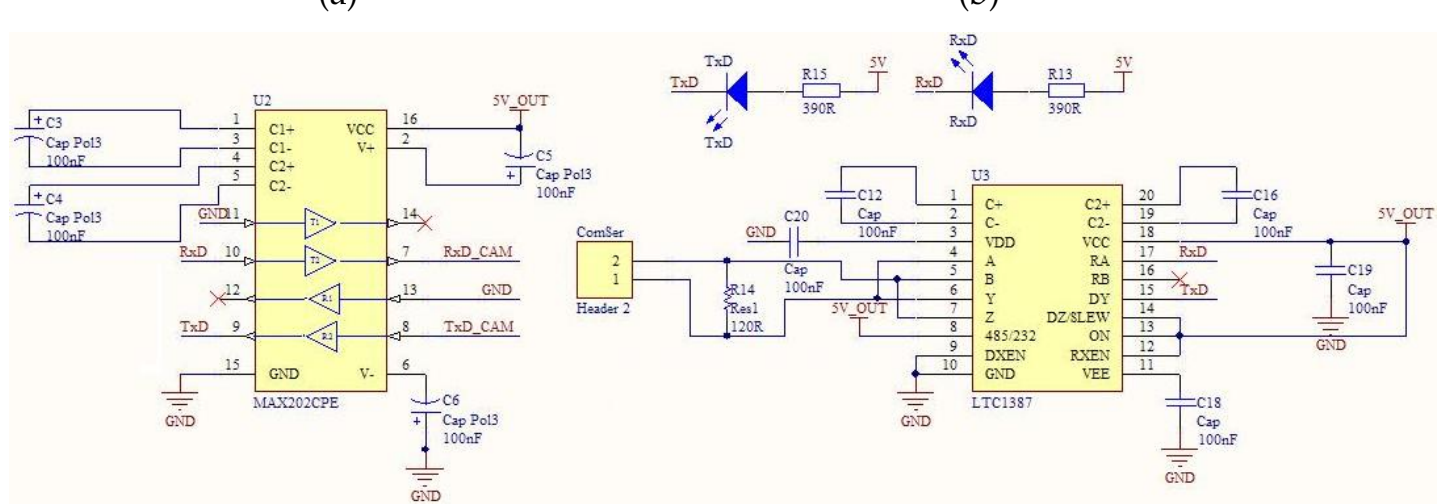

(c)
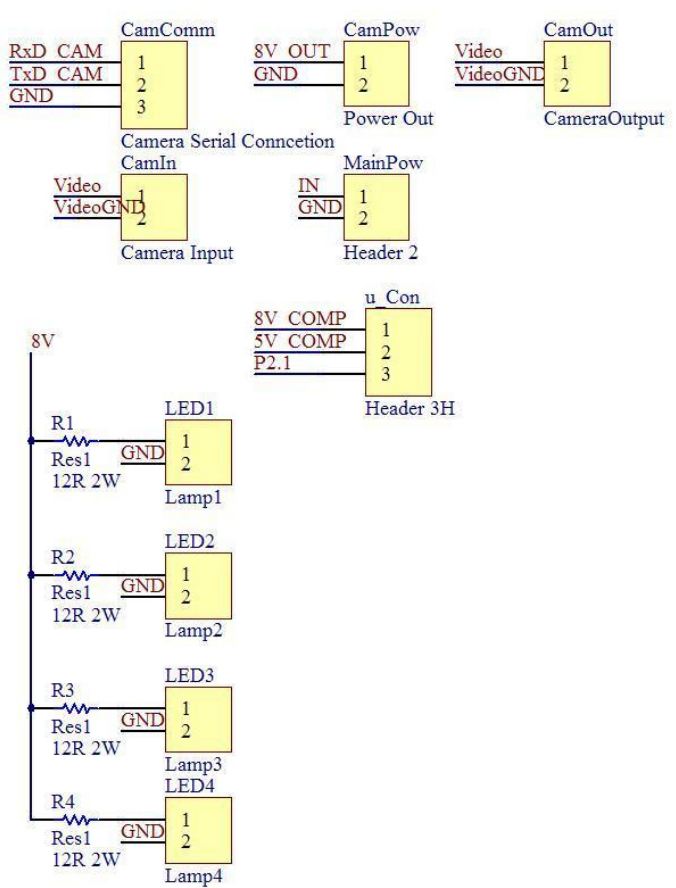

(b)

Figure 4.1: Commonalities between all camera board revisions include (a) the shape, (b) the inputs and outputs and (c) RS-232 and RS-485 communication circuitry. (c) the comparators for response to main control board.

puts (12 V, GND, RS485_A, RS485_B and control) and 4 unique outputs (Video, Video_GND, 8V_Comp and 5V_comp) (Figure 4.1(b)) .

For this board to handle the RS-485 communication for the camera, IC chips are included so that the RS-485 values can be converted to RS-232 protocol in order to be able to be read by the camera. The RS- 485 communication has to first be converted to TTL (Transistor-Transistor Logic) levels via a LT1387 chip and then converted to the RS-232 communication standard using the MAX202CPE IC (Figure 4.1(c)). A digital switch is also required so that the lights and camera 


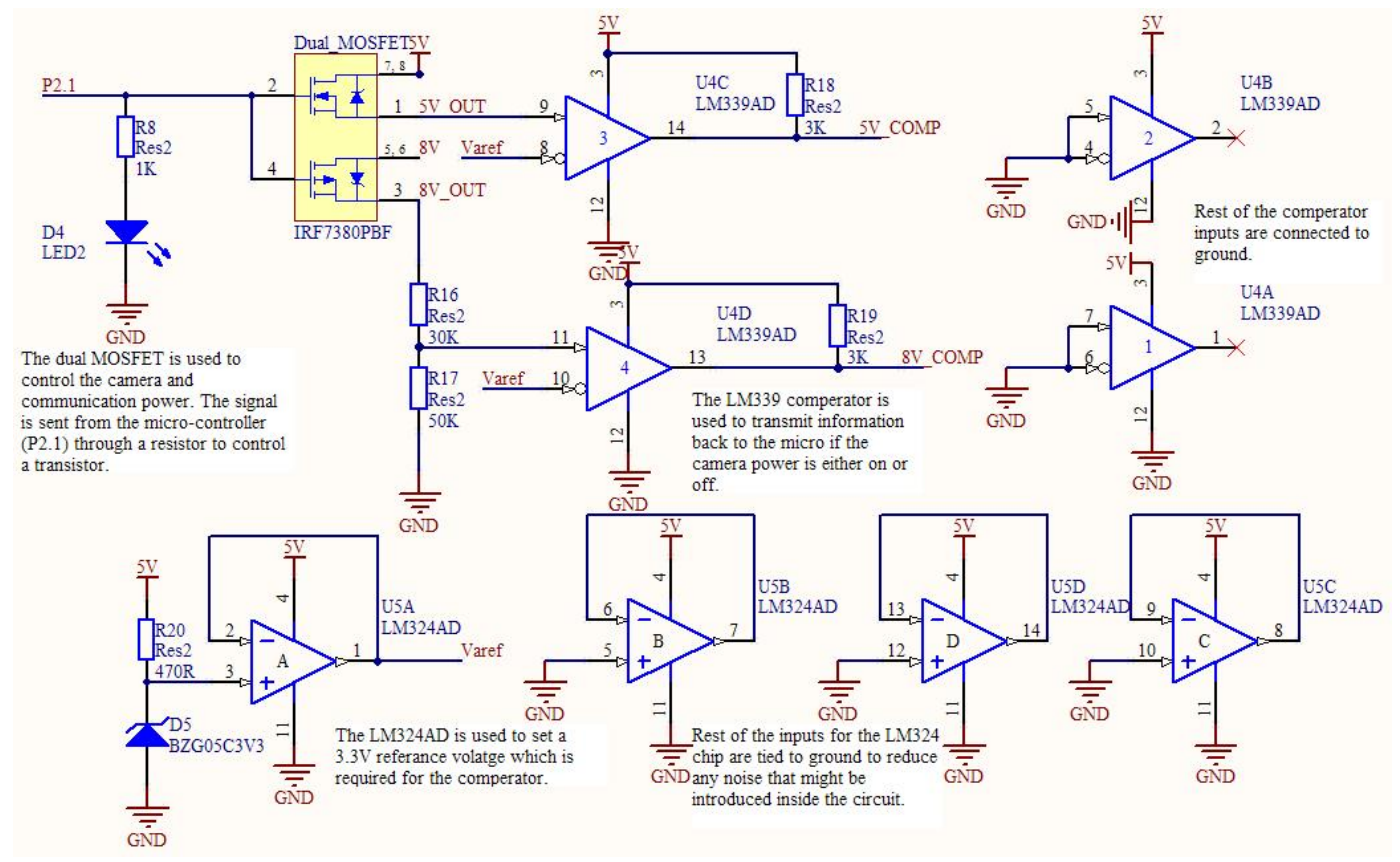

Figure 4.2: Comparators used on all camera boards for response to main control board.

can be turned on and off. The final components that are present on all board designs are the LM339 comparator for notifying the main control board if the lights and camera have successfully been turned on and the LM324 which is used to supply the $3.3 \mathrm{~V}$ reference voltage to the comparator so that it is able to distinguish between high and low values (Figure 4.2).

\subsubsection{Revision 1}

The first revision of the camera board (Figure 4.3) is a modification to that designed by Ristic. In this version the power input is taken from a $12 \mathrm{~V}$ line instead of the $24 \mathrm{~V}$ line as Ristic's previous design; and a surface mount regulator instead of a through-hole regulator is used as the surface mount version provides better thermal conduction and has the possibility of having a heat sink added. The through-hole version is unable to have a heat sink added as this would compromise the seals of the robot due to the size of the component. Greater thermal conduction is needed as the regulator has to convert the $12 \mathrm{~V}$ input down to $8 \mathrm{~V}$ with a possible $2 \mathrm{~A}$ throughput due to each of the four 


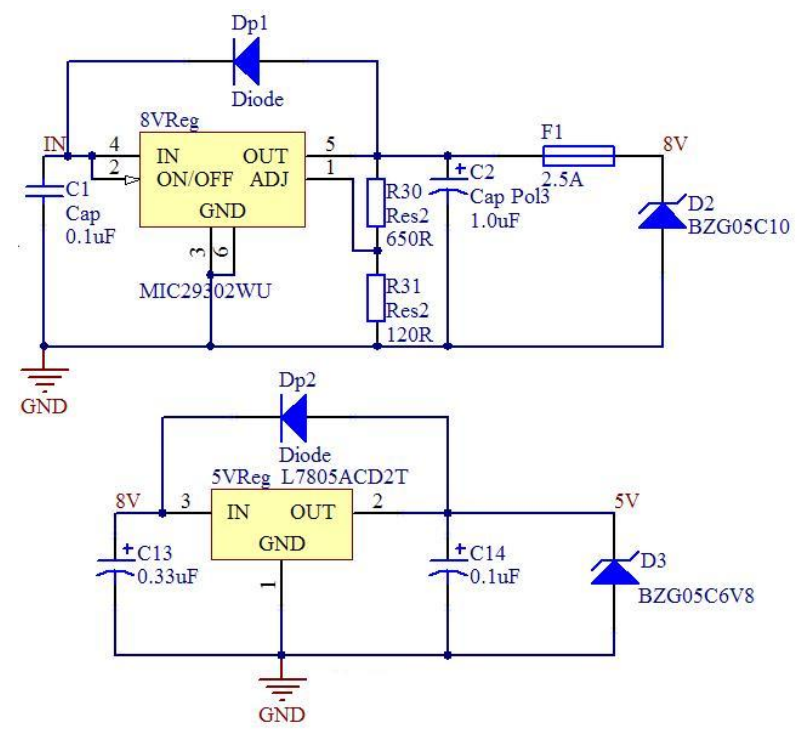

Figure 4.3: Camera board revision $1,8 \mathrm{~V}$ and $5 \mathrm{~V}$ voltage regulators required for camera, LEDs and IC chips.

LEDs drawing $0.35 \mathrm{~A}$, the camera drawing up to $0.5 \mathrm{~A}$ and the ICs on the board drawing a maximum of $0.1 \mathrm{~A}$. The regulator used for this is the MIC29302WU (Figure 4.3 top) 3 A linear adjustable regulator. This comes in a D2-PAK-5 case style which is a 5 pin surface mount package with a ground tab. The default pin mapping for this component in Altium was incorrect and thus needed to be modified so that the correct pins align with the correct function.

Due to the regulator drawing a maximum of $2 \mathrm{~A}$ of current and having a $4 \mathrm{~V}$ drop, $8 \mathrm{~W}$ of power is dissipated as heat at start-up. By adding a heat sink to the regulator a short term fix for this problem was accomplished. Ristic's design included four $2 \Omega, 2 \mathrm{~W}$ resistors which connect the LEDs to the $8 \mathrm{~V}$ power line. In the revised design, these resistors overheated and de-soldered themselves from the PCB because the LEDs are rated at $5 \mathrm{~V}$ (drawing an average of $0.35 \mathrm{~A})$ and the resistors have to accommodate the $3 \mathrm{~V}$ drop. Thus each resistor must continually dissipate $1.05 \mathrm{~W}$ of power without a heat sink (due to the size constraints). 


\subsubsection{Revision 2}

In this revision of the board both the $5 \mathrm{~V}$ and $8 \mathrm{~V}$ regulators have been replaced with switch mode regulators as these are more efficient and dissipate less heat. Both of these regulators take their supply from the $12 \mathrm{~V}$ line to reduce the current going through the $8 \mathrm{~V}$ regulator. The LEDs are now connected to the $5 \mathrm{~V}$ supply instead of the $8 \mathrm{~V}$ supply to reduce the power dissipated as heat. This also has the possibility of making the $2 \Omega, 2 \mathrm{~W}$ resistors redundant. Both of the regulators used are premade DC-DC converters which include the regulator IC, the capacitors and the inductor required for a switch mode regulator. The regulators used are from the PT78ST series DC-DC converters constructed by Texas Instruments (connection circuitry and PCB location shown in Figures
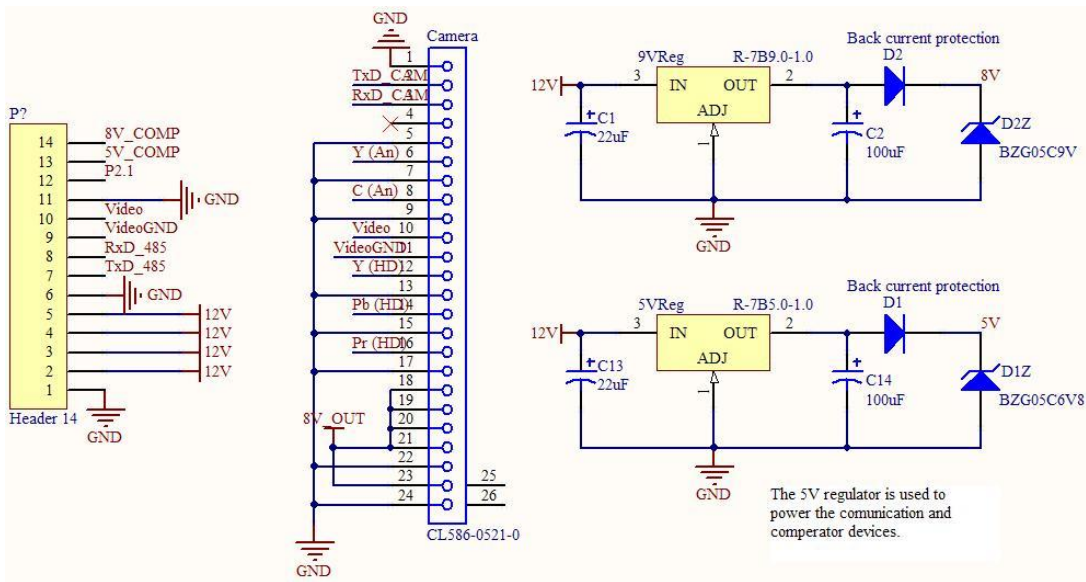

(a)

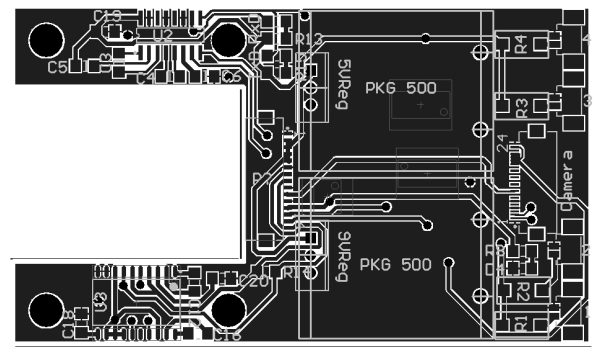

(b)

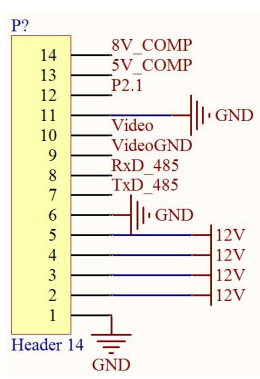

(c)

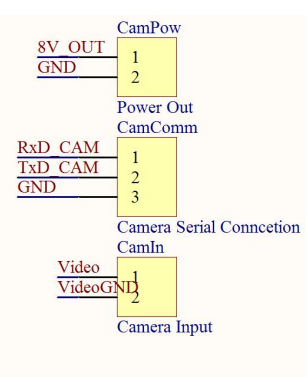

Figure 4.4: Camera revision 2 (a) schematic changes in power supplies and additions for the new camera, (b) top layer of PCB layout of new board showing central location of power supplies and (c) plugs kept on second version of board for the old camera. 
4.4(a) and 4.4(b) respectively).

Finally a single input connection is used to make replacement of the board easier and to reduce the likelihood of wires breaking. This connection is a 14 pin FFC (Flat Flexible Cable) connector which is a surface mount socket for a thin parallel cable with 14 lines and a pitch of $1.0 \mathrm{~mm}$.

Two versions of this board have been made, the first being designed to run the newly acquired Sony FCB-H11 colour camera module which uses a 24 line FFC for all its inputs and outputs (Figure 4.4(a)), including power and the multiple output options explained in section 6.2.1. For this, a 24 pin FFC connector with $0.5 \mathrm{~mm}$ pitch had to be added to the board so that it was possible to interface to the camera.

The second version is designed to interface to the old camera that is known to work. This requires retaining three of the 2-way headers so that the power, control signal and video feed can be supplied and retrieved (Figure 4.4(c)).

\subsection{Main Control Board}

The main control board controls all devices inside the base of the robot. This board is designed to understand the RS-485 communication signals sent from the computer via the base station board (section 5.1) and perform the appropriate function for each of these commands. The board must also provide power to all components inside the robot, hence this board is required to transform the $24 \mathrm{~V}$ output of the DC-DC power module (section 4.4, Vicor converter hereafter) to a $12 \mathrm{~V}$ supply to run both the camera motors and supply the camera board with power. It is also required to transform the $24 \mathrm{~V}$ input to a $5 \mathrm{~V}$ output to power the microcontroller and all onboard ICs.

Summing these requirements, the board must contain voltage regulators to 


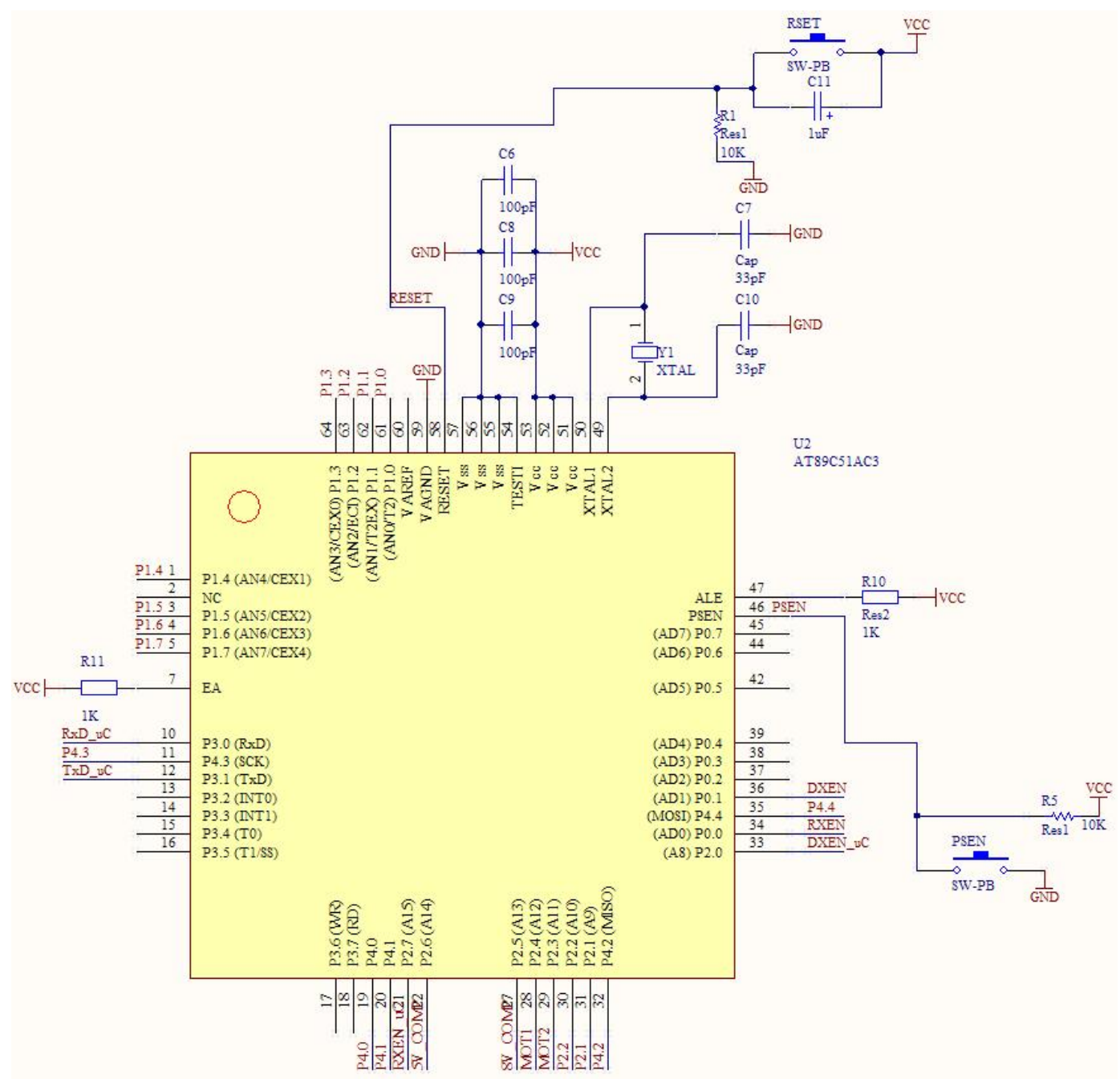

Figure 4.5: Main Control Board microcontroller and required components.

convert the $24 \mathrm{~V}$ input to the appropriate output values, a microcontroller so that components are able to be controlled (Figure 4.5); RS-485 communication circuitry so that the main control board is able to communicate with the base station board; RS-232 communication circuitry so that the microcontroller is able to be programmed (Figure 4.7); and the appropriate input and output connections for all off-board components (Figure 4.6).

The microcontroller used is the Atmel AT89C51ac3 8 bit 64 pin surface mount microcontroller. This was selected since it was used in the previous design and contains all of the functions required to fulfil the above requirements. Advantageously this author has considerable familiarity with it. 


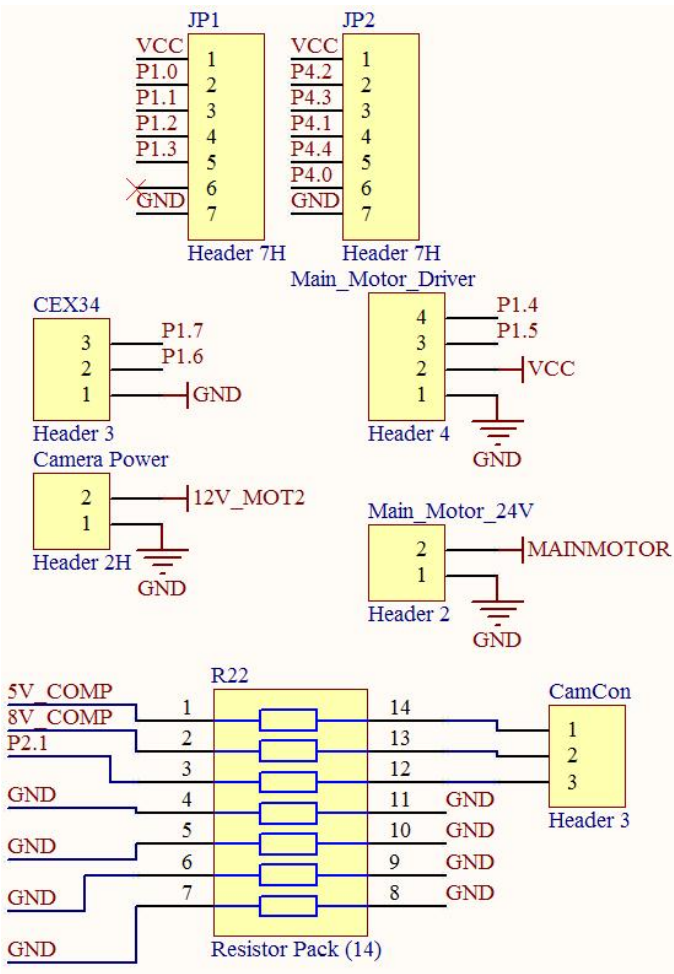

Figure 4.6: Main Control Board input and output ports.
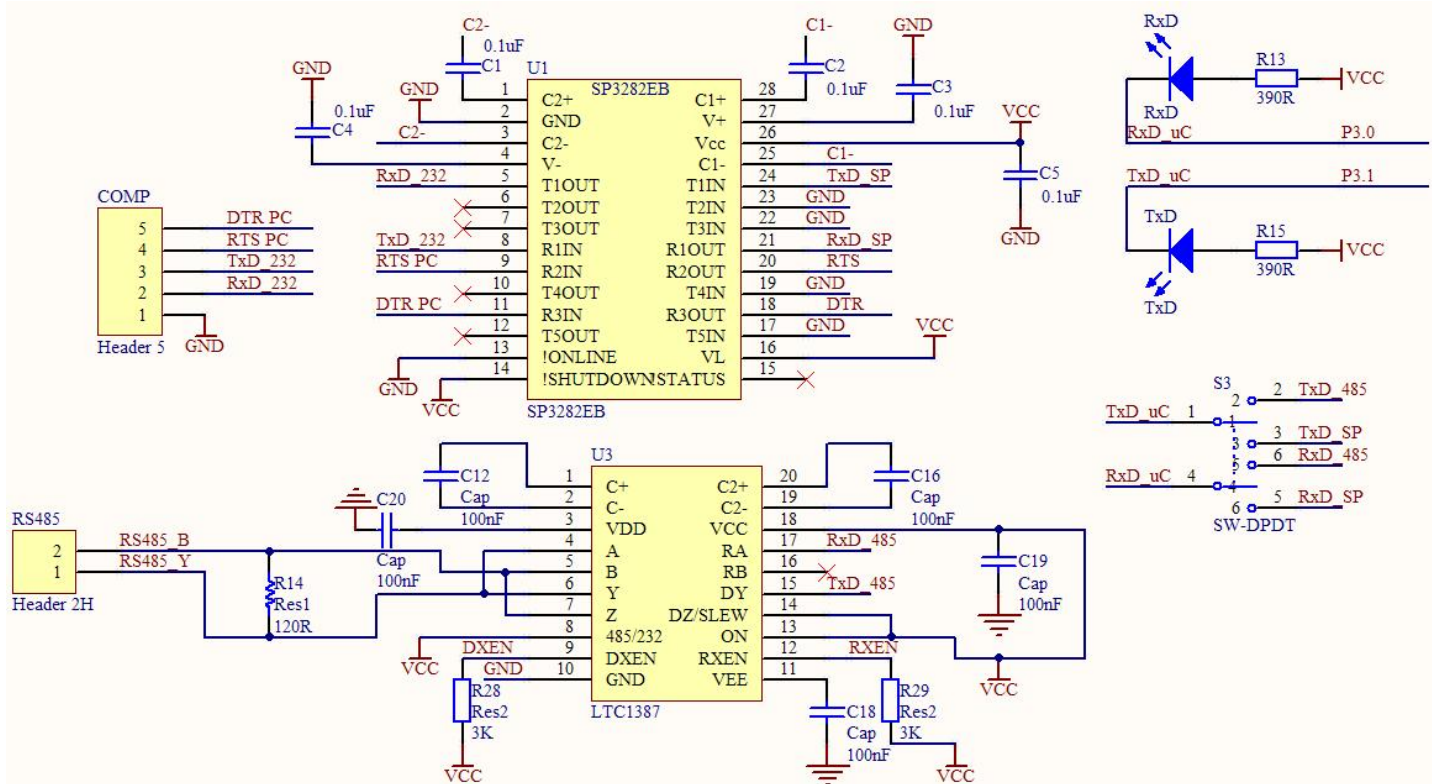

Figure 4.7: Main Control Board communication circuitry. 


\subsubsection{Revision 1}

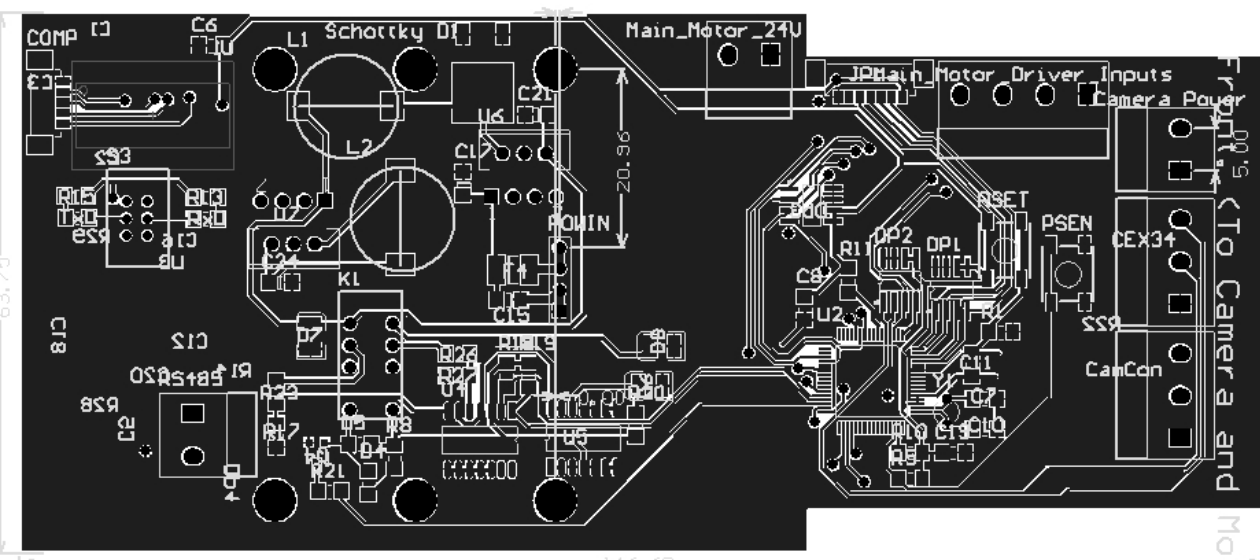

Figure 4.8: PCB layout of revision 1 displaying board shape.

Revision one (Figure 4.8) is a modification to the board designed and created by Ristic. The objective of this revision is to replace the linear voltage regulators with switch mode regulators (Figure 4.9) as the conversion of $24 \mathrm{~V}$ to $12 \mathrm{~V}$ with linear regulators causes $50 \%$ of the input power to be lost as heat. With this heating in a non-ventilated area, the components overheat and fail. This is most apparent at start up when the camera control board draws $2 \mathrm{~A}$ and hence $24 \mathrm{~W}$ of power is being dissipated through that one regulator.

The regulators chosen for this are LM2676T $12 \mathrm{~V}$ and $5 \mathrm{~V}$ variants. The $12 \mathrm{~V}$ version requires an input capacitance of $66 \mu \mathrm{F}$ and an output capacitance of $200 \mu \mathrm{F}$. For $12 \mathrm{~V}$ regulation a $33 \mu \mathrm{H}$ inductor is required to store the charge during switching. The values of the capacitors and inductors stated were de-

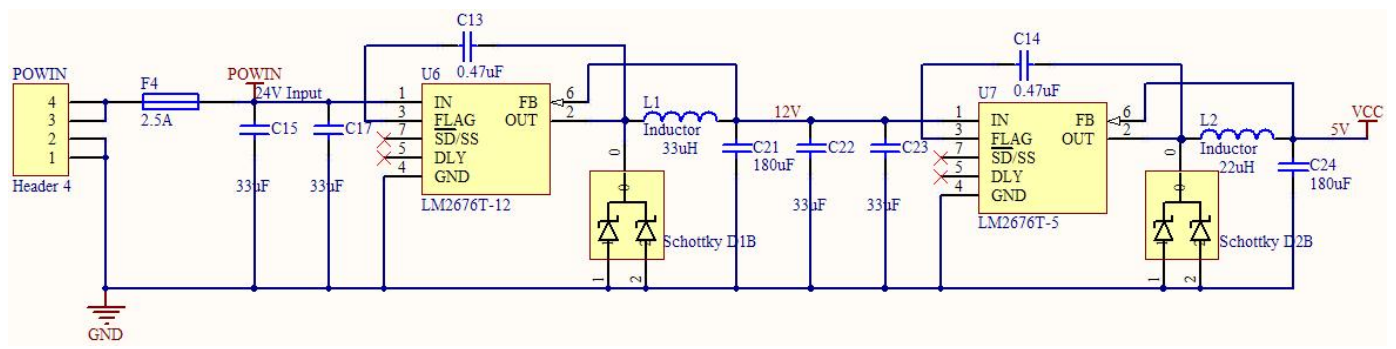

Figure 4.9: Main Control Board revision 1 power regulation circuitry. 
termined using the LM2676T datasheet provided [25], and for best regulation low ESR capacitors are used. A dual Schottky 6CWQ03FN 30 V 3.5 A diode constructed by Vishay (formerly International Rectifier [26]) is required for a rectified output voltage. Schottky diodes are used as they have a fast response time and a low voltage drop.

The LM2676T $5 \mathrm{~V}$ variant also requires an input capacitance of $66 \mu \mathrm{F}$ but it requires an output capacitance of $300 \mu \mathrm{F}$ and a $22 \mu \mathrm{H}$ inductor. This regulator is connected to the $12 \mathrm{~V}$ supply line not connected to the motors.

Another modification was to directly mount the main control board onto the Vicor converter (section 4.4) and connect to the power module board (section 4.4) as well. This was done by recreating the mounting hole design of the Vicor converter on the main control board. From this it is possible to precisely position the input plug in the same location as the output plug on the power module board as shown in Figure 4.10. The six large vias in Figure 4.10 are the recreation of the Vicor converter mounting holes, and the 4 pin "Power In" header is the connection to the power module board.

The final modification made in this revision was to replace the relay (Figure 4.11) designed to turn the motors on and off with a dual power MOSFET. The relay had to be replaced as it is rated at $3 \mathrm{~A}$ maximum current whereas the main motor is able to draw 4.2 A maximum when running at $24 \mathrm{~V}$. The use of

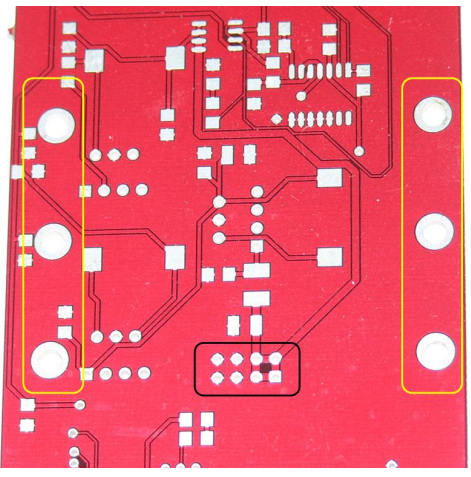

Figure 4.10: The location of the power input (Black) and mounting holes (Yellow) for the main control board. 


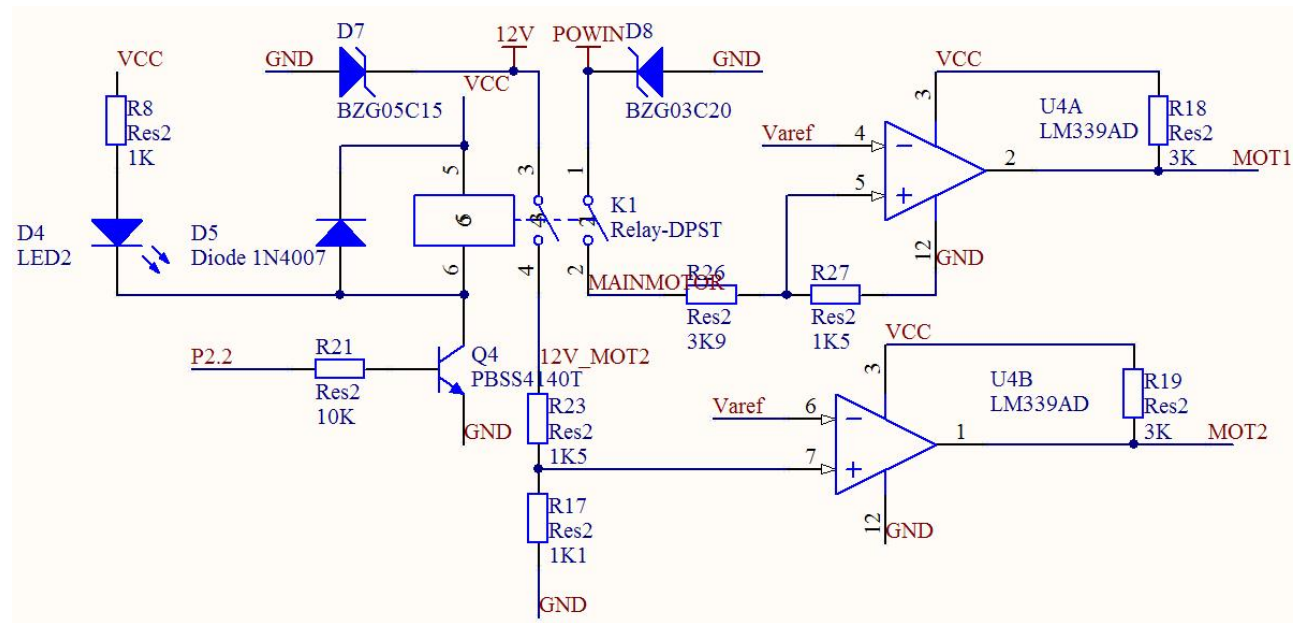

Figure 4.11: Main Control Board revision 1 motor power on and indication circuitry.

a FDS6982 dual power MOSFET with an inbuilt 8 A and 6 A power MOSFET is a better solution because of the current rating as well as its small size.

Two objectives were accomplished during this revision, the first being an increased understanding of the internal wiring, dimensions and mechanics of the robot; what circuitry and tasks are required for this system; and the tasks that the circuitry designed by Ristic preform. The second objective was overcoming some of the limitations present in Ristic's design.

\subsubsection{Revision 2}

Four modifications are made to the board in this revision, the first of these is to move all the input and output plugs on the board to one end so that when it is attached to the robot's lid it is easier to attach the wires connected to the components housed in the robot's base. These components are the 3 motors, the camera control board, and the connections to the base station board (input power, RS-485 communication and video feed). The second modification is to add an additional LM2676T-12 $12 \mathrm{~V}$ switch mode regulator and its corresponding circuitry (Figure 4.12). This is required as the overall current drawn from the $12 \mathrm{~V}$ line exceeds the regulator's 3 A rating. The camera head mo- 


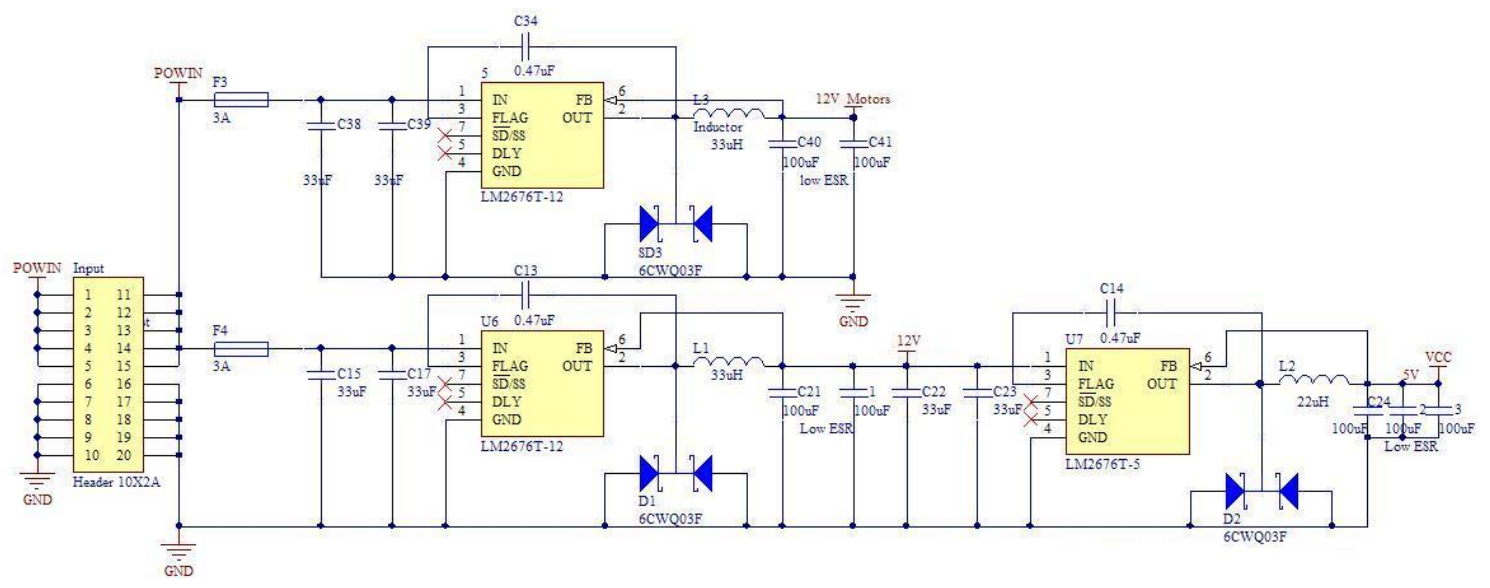

Figure 4.12: This schematic shows the use of two $12 \mathrm{~V}$ and one $5 \mathrm{~V}$ variant of the LM2676T switch mode regulator.

tors with their corresponding drivers each draw a maximum of $0.8 \mathrm{~A}$ from this $12 \mathrm{~V}$ line, the components on the main control board draw up to $0.2 \mathrm{~A}$ via the $5 \mathrm{~V}$ switch mode regulator and the camera control board draws up to $2.5 \mathrm{~A}$ for the on-board components, the camera and the LEDs. As a total of 4.3 A needs to be drawn from this $12 \mathrm{~V}$ line the supply is split into two parts; one line to power the motors and one line to power the on-board components and camera control board. It has been split this way so as to isolate the noisy motors from the digital components.

The third modification to the board is to add RC low pass filters between the microcontroller PWM output and the motor driver input as these motor drivers are designed to run off an analogue input signal. Using a RC filter network with a resistor value of $10 \mathrm{k} \Omega$ and capacitor value of $0.1 \mu \mathrm{F}$ allows for reasonable smoothing whilst not reducing the apparent response time of the motors.

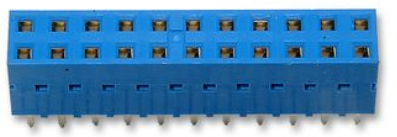

Figure 4.13: Illustration of type of power input plug, this is a 22 pin version as images of 10 pin versions are unavailable. 
The final modification to the board is to increase the size of the power throughput header that connects to the power module board. The current drawn by all of the components connected to the $24 \mathrm{~V}$ line exceeds that which the implemented 4 pin header can carry, hence a 20 pin header is used to allow for 10 input and 10 output pins, each line being able to carry 1 A (similar to Figure 4.13).

\subsubsection{Revision 3}

In this revision, the tasks that the main board must complete are split between two boards. This is done to overcome the wiring problems encountered when attaching the second revision to the robot. The first board continues with the name of "main control board" as it still performs the control tasks that are required, whereas the second board has been named "plug board" (section 4.3) as its only function is to provide the plug connections. The additional benefits are that the internal wiring of the robot can be managed and component replacement is easier as it is now possible to plug the lid of the robot into, and unplug the lid from the base of the robot with ease.

This "Plug 'n' Play" design (Figure 4.14) requires careful placement of all interconnecting headers, of which there are five to provide inputs and outputs for the three motors, the camera control board, the power throughput that is passed to the Vicor converter, the motor encoder inputs and the cable taut sensor input. Of these, the camera and motor headers are both 20 pin headers so that the required current can be passed as well as all control signals. The power throughput connection is a 16 pin header mostly to provide a strong connection at that end of the board as well as reducing the overall resistance of the connection as the pins act as parallel resistor. The encoder input is a 14 pin header as each of the motor encoders is connected to a 7 way header on the board, one of which is ground and another being $5 \mathrm{~V}$. Finally the taut sensor 


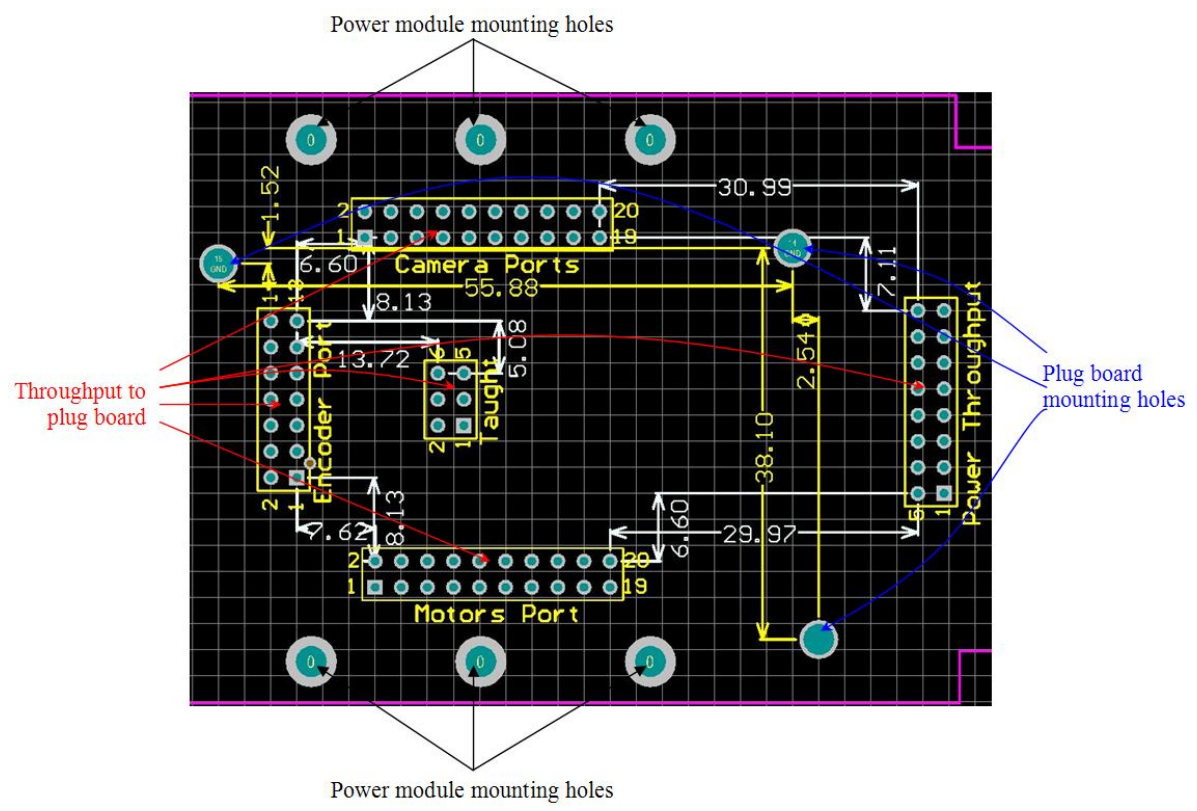

Figure 4.14: Main control board revision 3 shows the location of interconnecting plugs with dimensions. White and yellow dimensions relate to plugs and mounting holes respectively.

input header is a 6 way header which has a $5 \mathrm{~V}$ line, a ground line and a sensor reading line which goes high when the cable is tight. Each of these three connections are attached to 2 pins of the header to allow for easy pin mapping on the inverted plug board.

\subsubsection{Revision 4}

The fourth revision of the main control board is a version where the RS-232 interface used to program the board has been removed and replaced with a 10 pin header that connects to an external programming board. This has been done to reduce the number of components on the board and hence improve the reliability. The external board can be used to program both the main control board and the base station board (section 5.1). Additionally all screw terminals for the inputs and outputs of the motor drivers were replaced. The initial idea was to replace these with a 7 pin socket for the SyRen10 motor driver used to drive the main motor, and a 9 pin socket for the Sabertooth $2 \times 5$ motor driver used to control the two camera head motors (section 6.2.3). This idea was not 
implemented due to the limited current carrying capability of the wires able to be used with these plugs.

The plugs finally used are an 8 pin dual inline plug for the SyRen10 motor driver and a 10 pin version for the Sabertooth $2 \times 5$. These are selected as these plug and socket combinations accept wires from 16 to 22 AWG which allows for current carrying between $22 \mathrm{~A}$ and $7 \mathrm{~A}$ respectively [27]. The reasoning behind using plug and socket design is to allow for easy removal of components as well as easy management of all wiring inside the robot. Another advantage is that the two sockets require less space both laterally and vertically than the screw terminals which allows for more space for other components and boards (specifically the plug board in section 4.3).

Due to the possibility of device failure, an optocoupler is included to isolate the micro controller from the motor drivers. The isolation has been placed between the microcontroller PWM output lines and the RC filter used to convert the digital signal into an analogue approximation for the motor drivers. Thus if the motor driver fails causing the $12 \mathrm{~V}$ or $24 \mathrm{~V}$ input to be returned down the control line, the optocoupler will prevent this reaching the microcontroller and prevent additional damage to the system.

Another modification made to the main control board is the addition of a $5.4 \mathrm{~V}$ zener diode on the VCC $5 \mathrm{~V}$ line. This has been added as there is the possibility of the switching regulator circuits failing if one of the other components connected to it fails, such as happened with the dual n-MOSFET device when a voltage spike occurred on one of its pins. This device failed due to the $\mathrm{V}_{g s}$ voltage continually being applied to the device being at the limit of its maximum ratings, thus when the spike occurred, the device failed and allowed current to flow into the control pin. This error has been corrected by using a p-MOSFET as the board layout has the device as a high side driver which a n-MOSFET is not. To further prevent damage the n-MOSFET is over-specified. The Fairchild 
Semiconductor FDS4925BZ dual n-channel MOSFET is chosen as it is able to run continuously with a drain to source voltage of $30 \mathrm{~V}$ while supplying $6.9 \mathrm{~A}$ per MOSFET. Thus the device is able to run the main $24 \mathrm{~V}$ motor even when it stalls and draws $4.1 \mathrm{~A}$.

\subsection{Plug Board}

The plug board has been designed to make it easier for AES technicians to put the robot back together if a component needs to be replaced. This board also has the added advantage of helping manage all wiring inside the robot as it is housed in the base of the robot. The improvement is illustrated in Figures 4.15 below.

The board has no components on it, rather it contains plugs for all the wires that are inside the base of the robot and contains headers to transfer data to

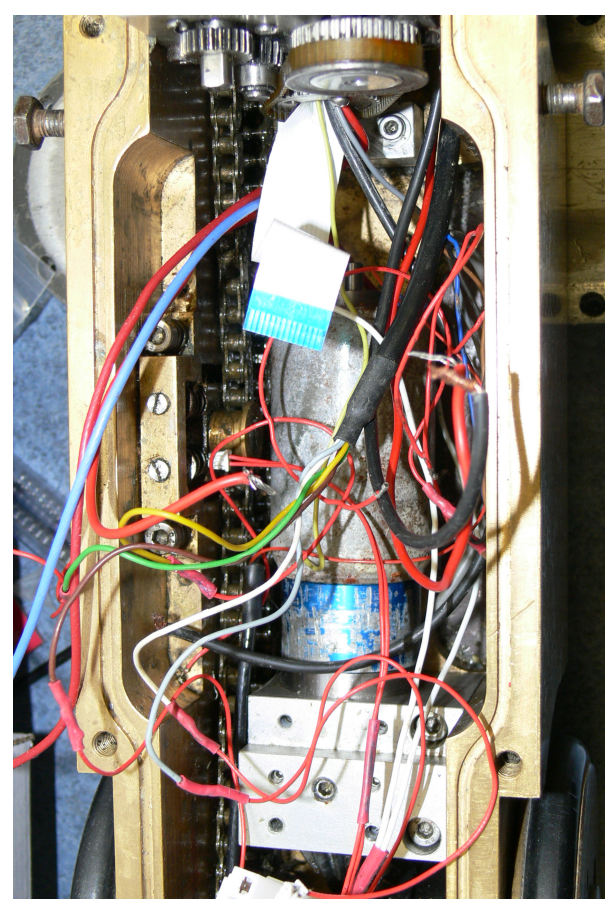

(a)

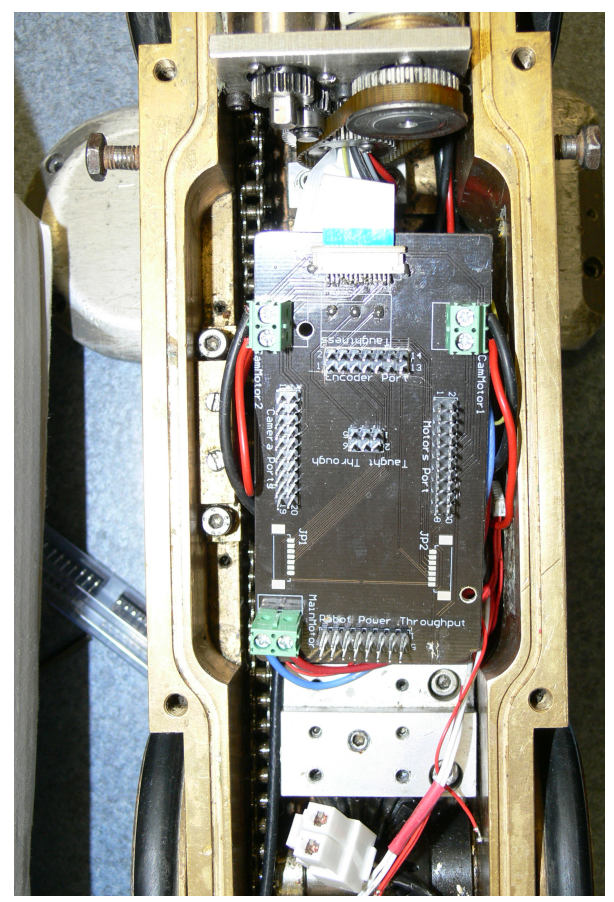

(b)

Figure 4.15: Wiring inside of robot (a) un-managed and (b) managed by the plug board. 
and from the main board, which it is directly attached to.

Screw holes also exist on the board so that stand-offs can be attached between the plug board and the main control board to lock the boards together so that they will not jolt free if the robot collides with an obstacle.

\subsubsection{Revision 1}

The first revision of the board included five headers to connect the board to the main control board (Figure 4.16), including two 20 pin headers for the motor control and the robotic head control throughputs as well as the LEDs and camera control signals. A 16 pin header is required for the robot's $300 \mathrm{~V}$ power throughput that is directly transferred to the Vicor converter by the main control board. The fourth is a 14 pin header that provides the data from the motor

From/To Micro

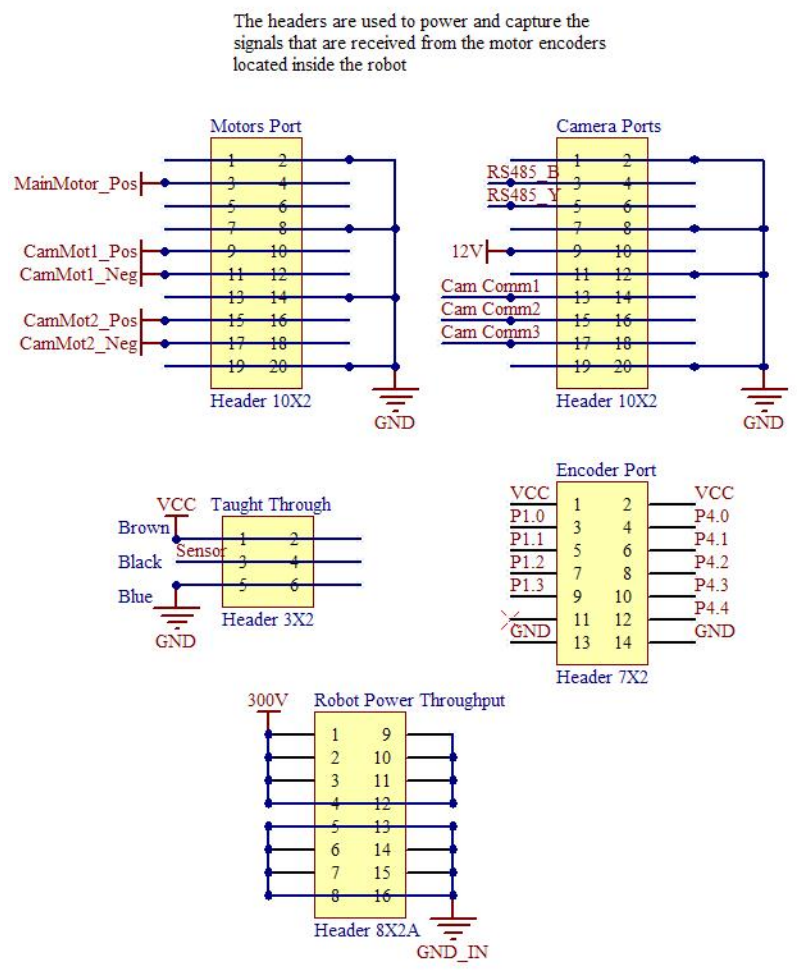

Figure 4.16: Plug board revision 1, I/O Headers for connection to the main control board. 


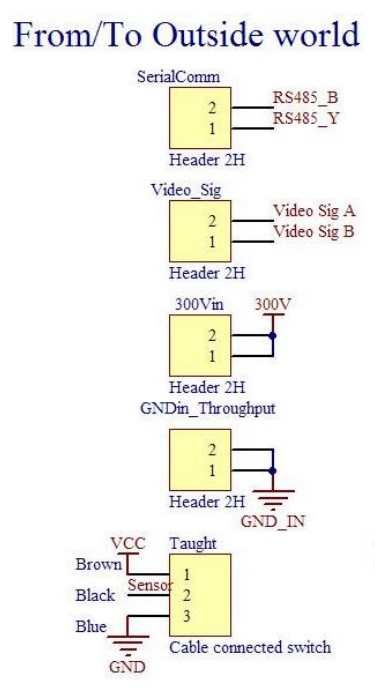

From/To Robot

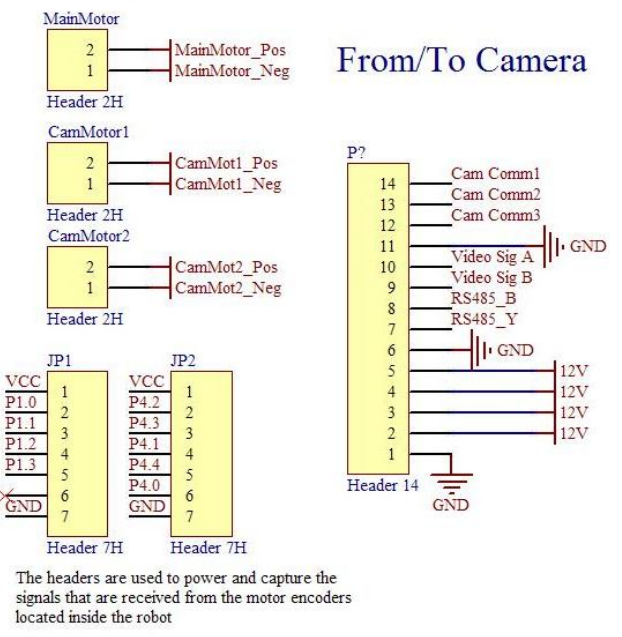

Figure 4.17: Plug board revision 1, I/O plugs for attachment to components connected through base of robot.

encoders. The final header is a 6 pin header for the cable sensor. This sensor is designed to trigger when the cable goes slack, sending a logic value to the robot's onboard micro causing the robot to stop reversing. The board therefore contains other inputs and outputs to correspond to these headers, including a 24 pin Flat Flex Cable connector for the camera head, two 7 pin encoder ports, three 2 pin motor outputs for the main drive motor and the two camera head motors, a taut sensor input and two 2 pin headers corresponding to the transmission down the cable (Figure 4.17).

For the PCB (Figure 4.18) to fit inside the robot and have all connections in the correct locations for their mates, careful consideration in the shaping and layout is required. The board is similar in shape to the main control board other than the side has to be cut straight to allow room for the chain connecting the front and rear wheels (outlined in section 2.2). 


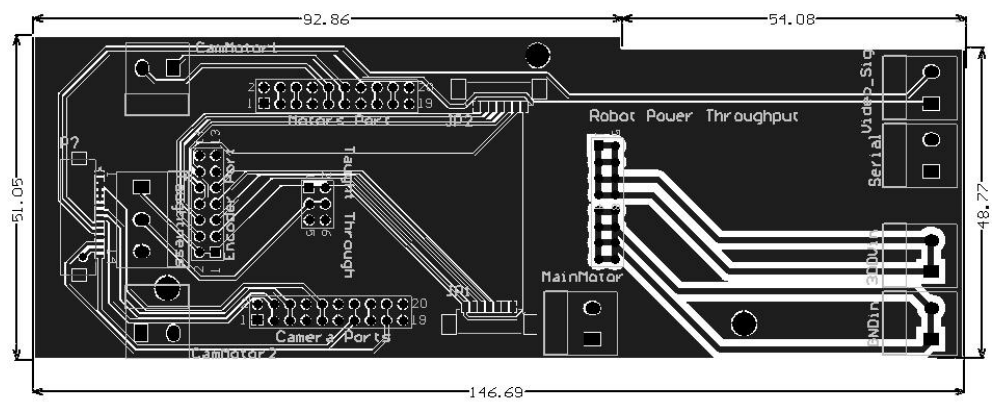

Figure 4.18: Plug Board revision 1, PCB layout displaying large tracks for power throughput and board size.

\subsubsection{Revision 2}

A second revision of the plug board was required as the internal measurements of the robot were taken from the main motor and not the mounting frame used to hold this motor to the chassis of the robot. This frame holds the main motor and its bevel gears securely in place.

The board therefore needed to be shortened as the space at the back end of the robot will only allow for one board above the motor holder. The original design had allowed for $30 \mathrm{~mm}$ of clearance throughout the inside of the robot. The main control board extrudes $12 \mathrm{~mm}$ out from the lid of the robot, the standoffs separating the main board and plug board are $15 \mathrm{~mm}$ long to allow for
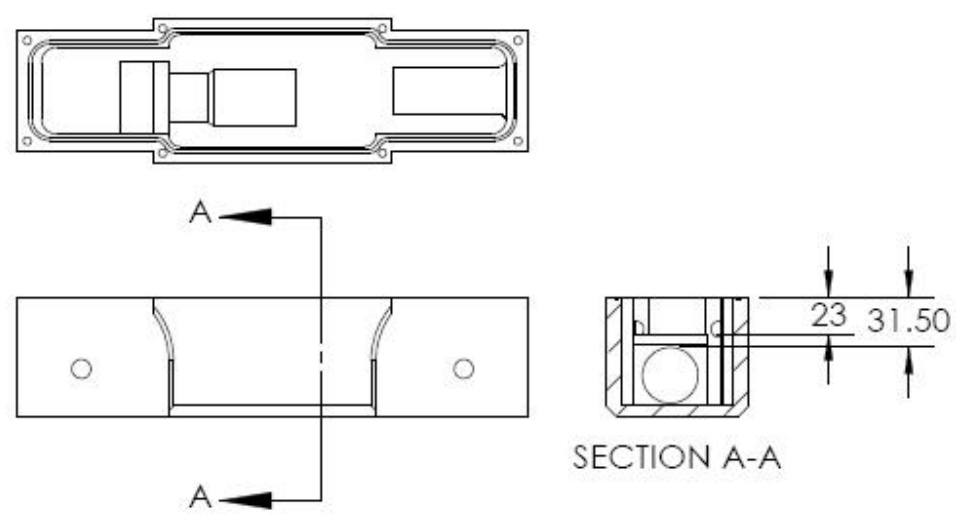

SECTION A-A

Figure 4.19: Drawing of the base section of the robot with main drive motor and cradle inside displaying distances (in $\mathrm{mm}$ ) between top of base section and the cradle as well as from the top to the motor. 


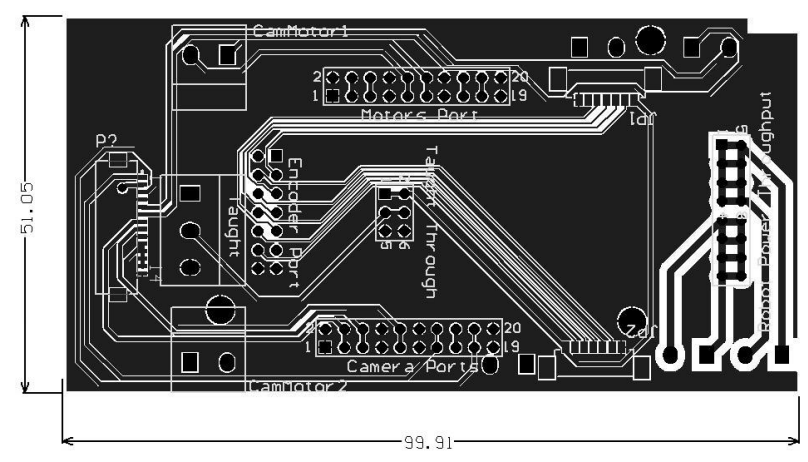

Figure 4.20: Plug Board Revision 2 PCB layout showing that the design has been reduced in size to fit inside the robot by cutting off the right hand side.

clearance for the input and output terminals of the boards, and the PCBs are $1.5 \mathrm{~mm}-2.0 \mathrm{~mm}$ thick, thus $29 \mathrm{~mm}$ of clearance is required for the boards to fit inside the robot. However as shown in Figure 4.19, there is only $23 \mathrm{~mm}$ of clearance above the motor cradle, therefore there is not enough room for both boards and the separation of $15 \mathrm{~mm}$ between them.

This revision therefore consisted of shortening the plug board so that the end would stop before reaching the motor cradle. The board still contains the same inputs and outputs as the first revision.

Figure 4.20 shows the new plug board shape which has been cut to stop before reaching the motor cradle. The input and output plugs connecting the robot to the cable need to be relocated as that end of the board has been removed. The possible location of these plugs is now in the same vicinity as the plugs on the main control board hence the fourth revision of the main control board (section 4.2.4) was required to make room for the plug board.

\subsection{Power Module Board}

The robot contains a Vicor V300B24C250B power module (Figure 4.21(a), acquired from Powerbox Australia [28]) for conversion of the input voltage to a usable output. This module is a Vicor $250 \mathrm{~W}$ DC/DC converter that takes 


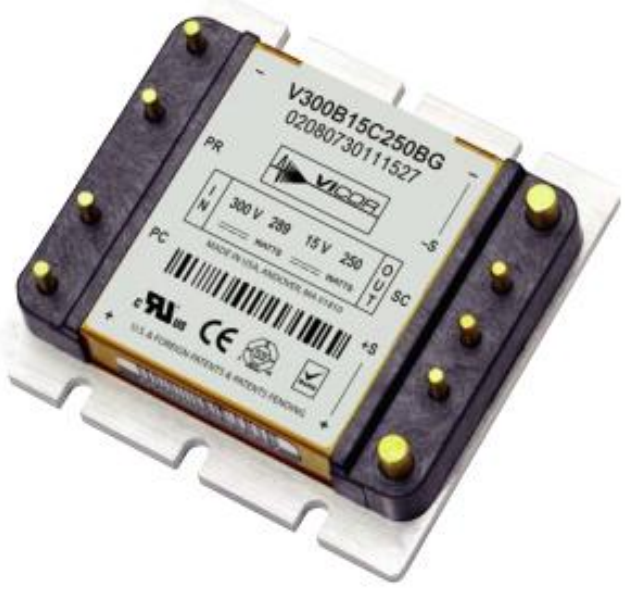

(a)

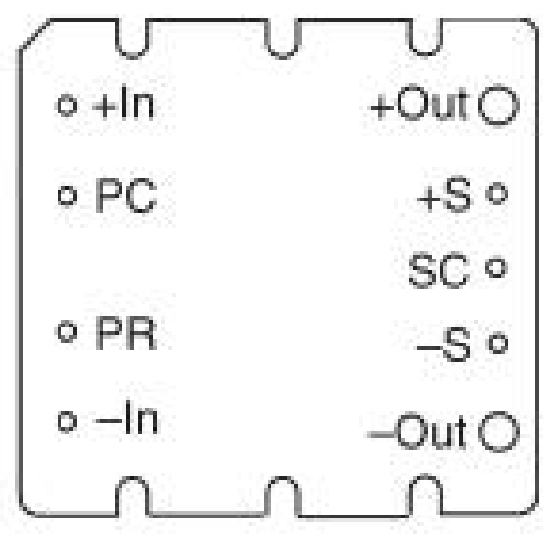

(b)

Figure 4.21: (a) Vicor 300V Input Mini Family DC-DC converter, (b) Pinout of DC-DC converter

the $300 \mathrm{~V}$ input from the base station and converts it to $24 \mathrm{~V}$ with capacity to provide 10.4 A through the connected circuitry. The converter has dimensions of $57.9 \mathrm{~mm} \times 55.9 \mathrm{~mm} \times 12$. It has 9 pins, 4 on the input side and 5 on the output side, of these 6 are used; the positive and negative input connections, the positive and negative sense connections and the positive and negative output connections as displayed in Figure 4.21(b).

The power module board is designed to slot directly onto the Vicor converter and connect the sensor terminals to their corresponding outputs to stabilise the voltage. This is done as no DC-DC converter will be inherently stable for all possible loads, thus by creating an impedance loop the DC-DC converter voltage becomes more stable during operation [29].

The system designed by Ristic incorporated a $12 \mathrm{~V}$ Vicor DC/DC converter but due to this being unable to run the main motor at its specified speed, a $24 \mathrm{~V}$ version had to be implemented. While working at AES, Ristic designed and populated a PCB with screw terminals for inputs and outputs. This PCB includes a thermistor for in-rush current protection. 
The object of redesigning this board is to reduce noise introduced to the robot via the cable and to protect the circuitry of the robotic system and the module itself from voltage spikes.

\subsubsection{Revision 1}

In the first revision of this power module board; the thermistor that was previously on this board was removed and replaced with bypassing capacitors and TVS diodes on both the input and output sides of the Vicor converter (Figure 4.22). The capacitors reduce the noise on the system and the TVS diodes help remove voltage spikes from the power lines. The thermistor was removed as protection against inrush current is better handled on the other side of the AC to DC transformer which is housed in the base station and is therefore is not needed in this location.

The screw terminals for the output to the main control board have also been removed and a plug has been added to make insertion and removal of the boards easier. Therefore, the power module and main boards need to be positioned to ensure the boards can fit inside the robot and the plug will connect. This requires the output plug to be correctly aligned with the Vicor converter as the main control board is designed to mount to both of these. Using the manufacturer's specifications (Figure 4.23(a)) a working power module board was created (Figure 4.23(b)).

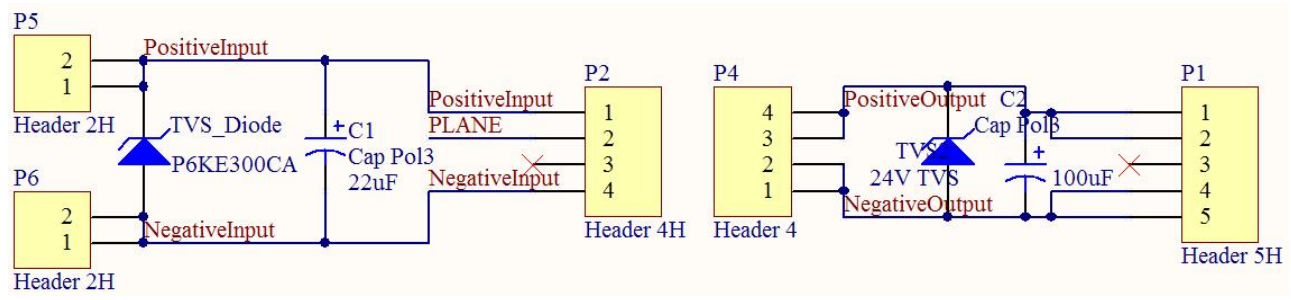

Figure 4.22: Power module board revision 1 schematic. 


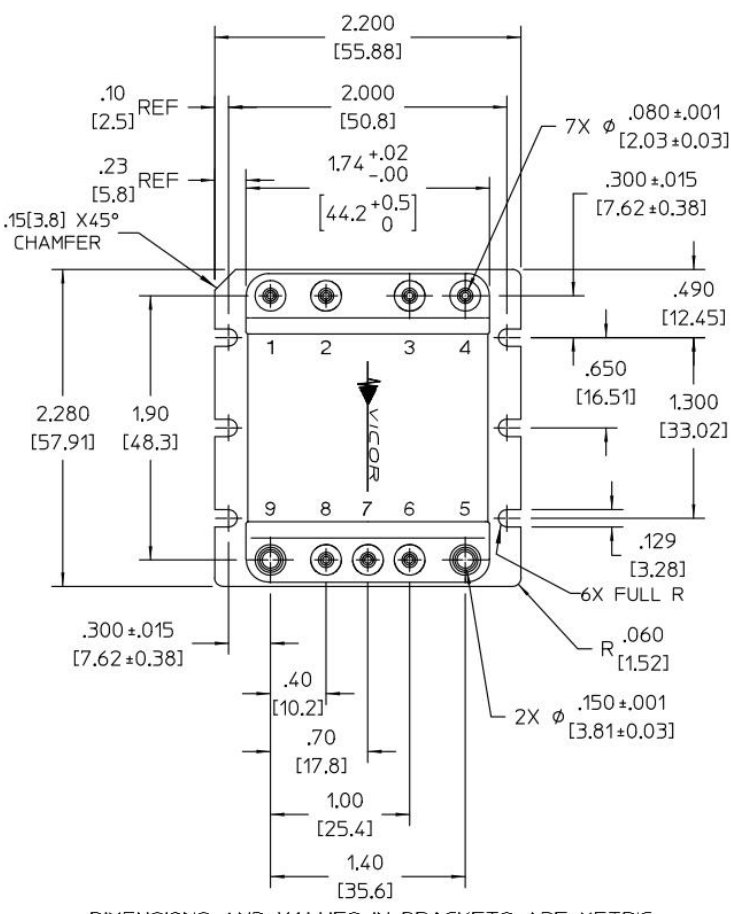

DIMENSIONS AND VALUES IN BRACKETS ARE METRIC.

(a)

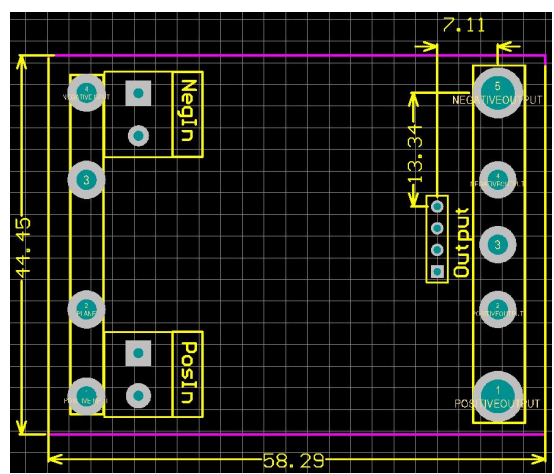

(b)

Figure 4.23: The (a) PCB layout dimensions used to position the header on the power module board so that it mates correctly with the main control board, positions are calculated with help from (b) manufacturer specifications.

\subsubsection{Revision 2}

The second redesign of the power module board increases the size of the output header to handle a larger - potentially $10 \mathrm{~A}$ - current throughput of the converter. To make this connection secure and to handle this large current, a

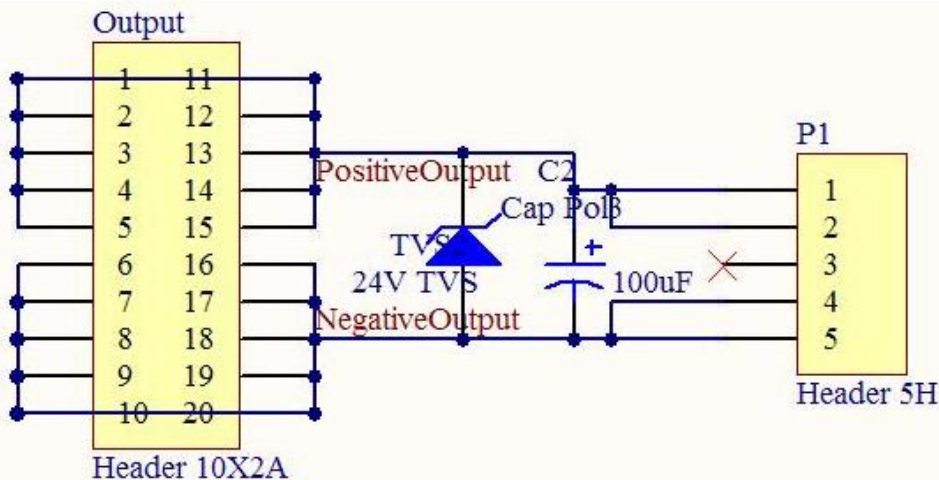

(a)

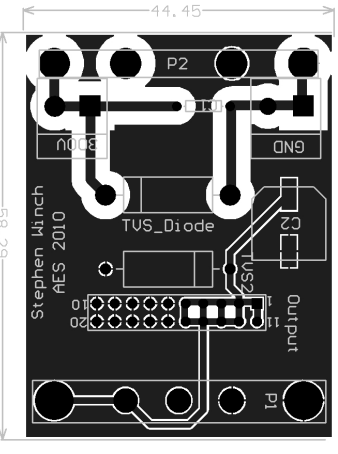

(b)

Figure 4.24: Power module board revision 2 (a) modification and (b) PCB layout showing new size and position of header. 
20 pin header has been used to provide ten $24 \mathrm{~V}$ and ten ground connections as shown in Figures 4.24(a) and 4.24(b). This was not completed in revision 1 as the current limitation of the header used was not stated on the supplier's website [25], and was discovered later through another supplier of the same product [30].

\subsection{Internal Mounting}

The brass casing of the robot is used as a heat sink for the Vicor DC to DC converter (Figure 4.25). This also provides a connection platform for all circuit boards inside the base of the robot.

Replacement of components has been made easier with the creation of a board stacking design using the Vicor converter as a base. This board stacking (Figure 4.26) provides reliable connections between the Vicor converter, main control and the plug boards and facilitates easy robot re-assembly by allowing the plug board to manage loose wiring before the lid is attached. The time taken to replace components has also been reduced by removing a large number of the screw terminals.

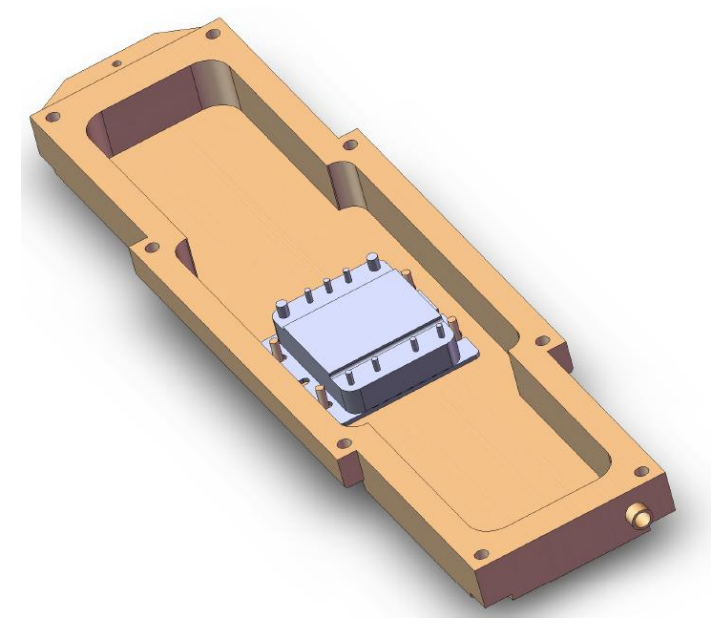

Figure 4.25: Robotic unit lid with Vicor DC to DC converter attached 


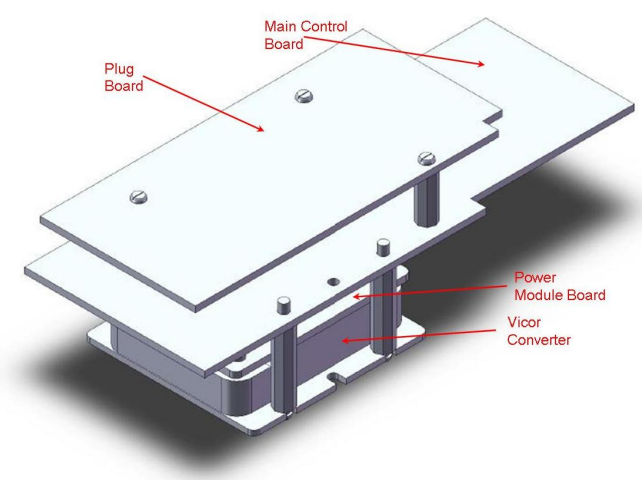

(a)

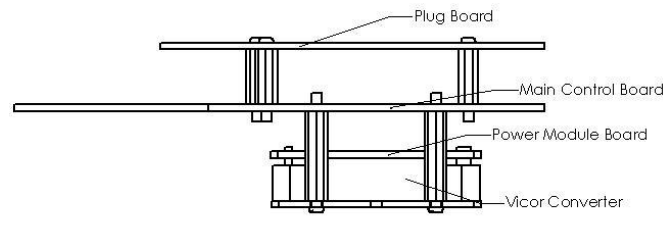

(b)

Figure 4.26: (a) Model of and (b) drawing of board stacking design used to securely fasten internal boards to the frame of the robot.

The camera board is the only internal board not to be directly connected to another board. It is connected to the plug board via a FFC which extends down the camera head shaft. The use of a singular cable for connecting the plug board to the camera board allows for easy replacement of components if one becomes damaged. The camera board is attached to the robot frame with the use of 4 screws (Figure 4.27).

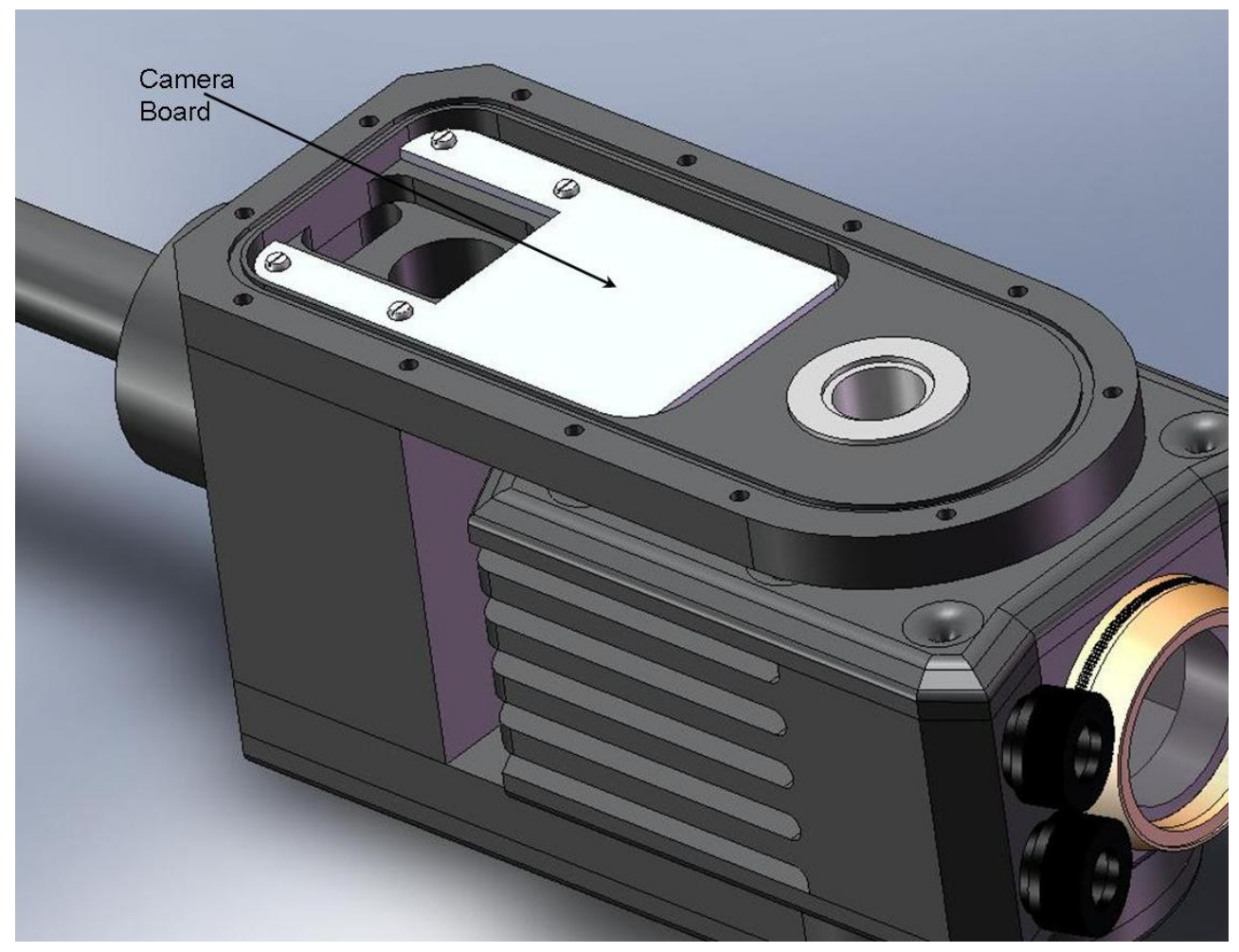

Figure 4.27: Camera board mounted inside robot's head with 4 screws. 


\subsection{Internal PCB Summary}

The replacement of the linear regulators which switch mode power supplies and the attaching of the Vicor converter to the frame has overcome the problem of overheating of the internal components. The switch mode regulators are more efficient than their linear counterparts, and hence the power consumption of the robot has been reduced (discussed further in section 8.1.1).

Replacement of components has been made easier with the creation of a board stacking design and the use of a singular cable for connecting the plug board to the camera board. Additionally, this board stacking provides reliable connections between the Vicor converter, main control and the plug boards and simplifies robot re-assembly by allowing the plug board to manage loose wiring before the lid is attached. The time taken to replace components has also been reduced by removing a large number of the screw terminals. Two versions of the camera board exist, one for each of the Sony cameras used in this project. These boards are small in size, low in profile and use commercial DC-DC stepdown regulators for convenience and ease of replacement.

All internals board have been completed with only minor modifications required to the camera board once the full functionality of the Sony FCB-H11 camera had been tested. The boards have been designed to fit inside the stringently defined dimensions of the robotic unit and to meet the requirements of AES. This required the selection of small and low profile IC packages whereever possible.

All of these internal boards are secured to the frame of the robot either directly in the case of the camera board or via the board stacking method (section 4.5).

Power throughput requirements from board to board and to connected devices has been taken into account and connectors have been over specified for reli- 
ability. Specifically header connections for power supplies between the plug, main control and power module boards have continuous current ratings significantly greater than that drawn by connected components. 



\section{Chapter 5}

\section{External Printed Circuirt Boards}

This section introduces four PCBs employed external to the robotic unit. This includes two boards designed for control at the base station of the system, one reprogramming board and one board designed for the testing of a newly acquired camera.

\subsection{Base Station Board}

The base station board is designed to take the input from the computer via USB, convert this signal to TTL logic levels for the onboard microcontroller and RS-485 standards so that it can be transmitted to the robot.

The base station controls the motorised cable reel and reads the encoder attached to the reel to indicate the distance that the robot has travelled. The motorised reel is controlled such that the robot can not run over its own cable and sever it.

Standard components on all base station redesigns are the AT89C51ac3 microcontroller (Figure 5.1(b)) and the FT232BL USB interface IC (Figure 5.1(a)) which converts the data transmitted by the computer to TTL logic levels. These 
signals are sent to the robot via a LT1387 IC (Figure 5.1(a)) which converts the TTL logic levels to RS-485 communications protocol for transmission down the $300 \mathrm{~m}$ long cable.

USB - TTL - RS485 Communication circuitry

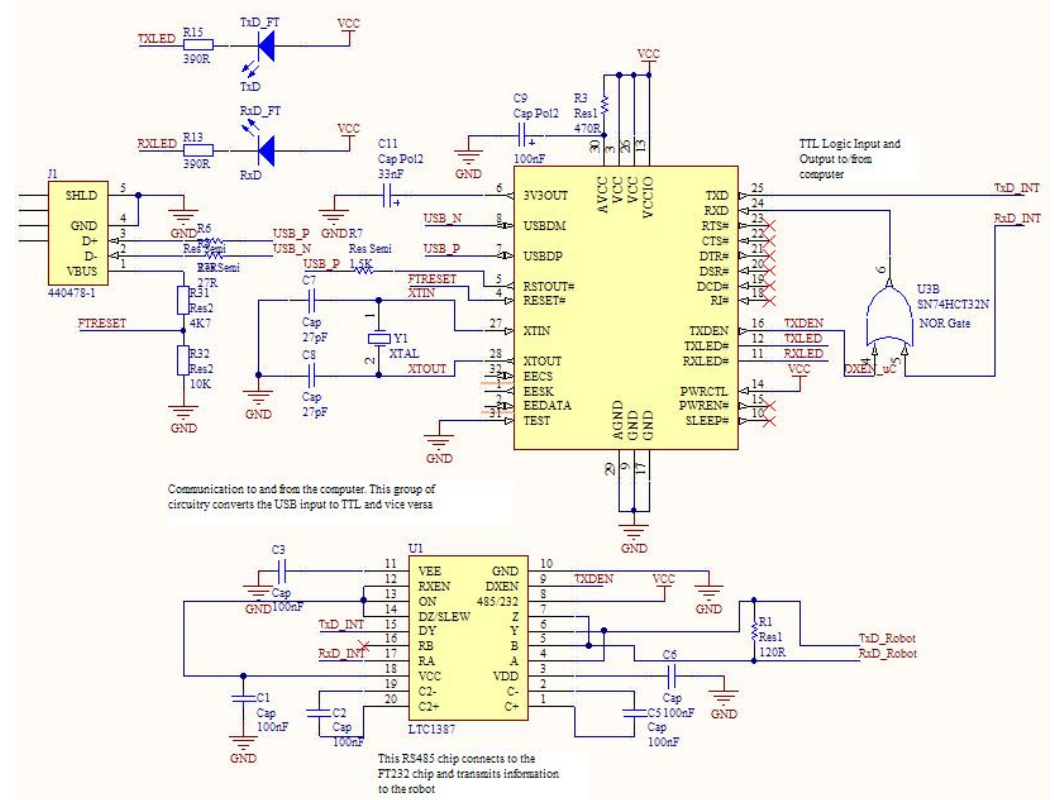

(a)

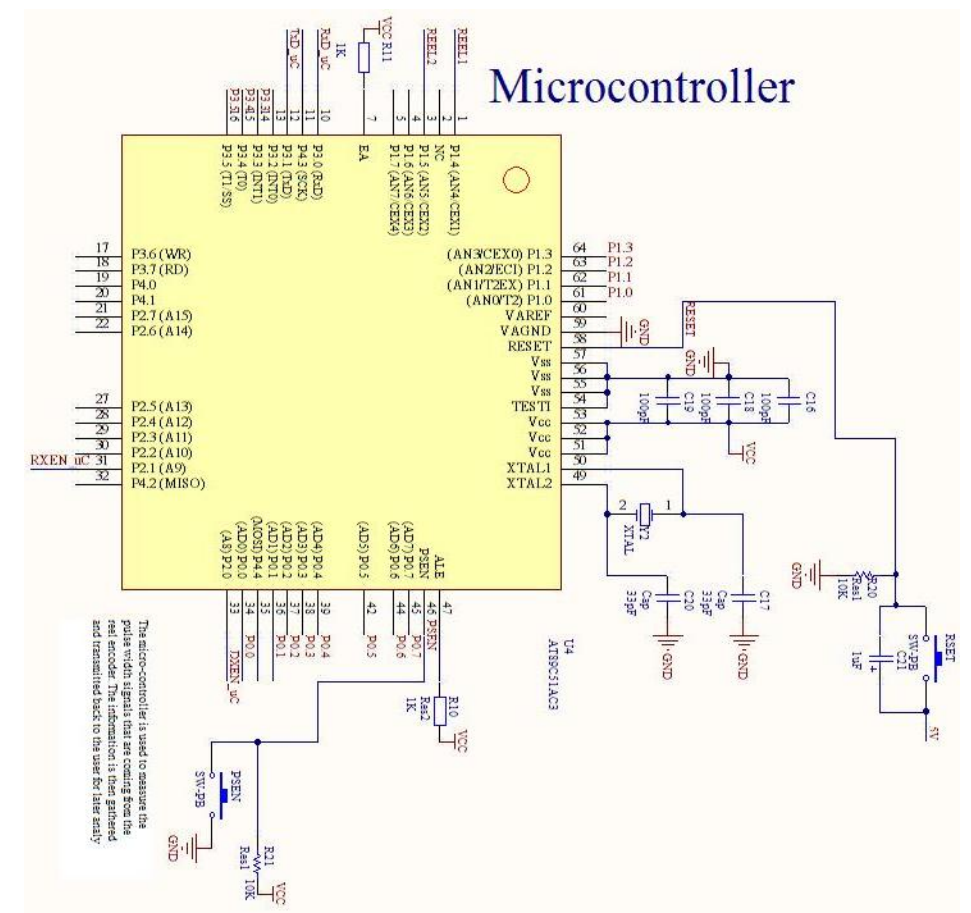

(b)

Figure 5.1: Common components on the base station board include the (a) Communication and (b) Microcontroller circuitry. 


\subsubsection{Revision 1}

The first revision of the base station board (Figure 5.2) is a modification to incorporate a power output for the reel motor and an onboard motor driver (Figure 5.2(a)) to run the motor required to control the reel. Neither of these were in Ristic's original design. The re-programming IC (Figure 5.2(b)) from Ristic's design was kept as was the input and output ports (Figure 5.2(c)) although protection was added to the voltage inputs.

\section{Re-programming circuitry}

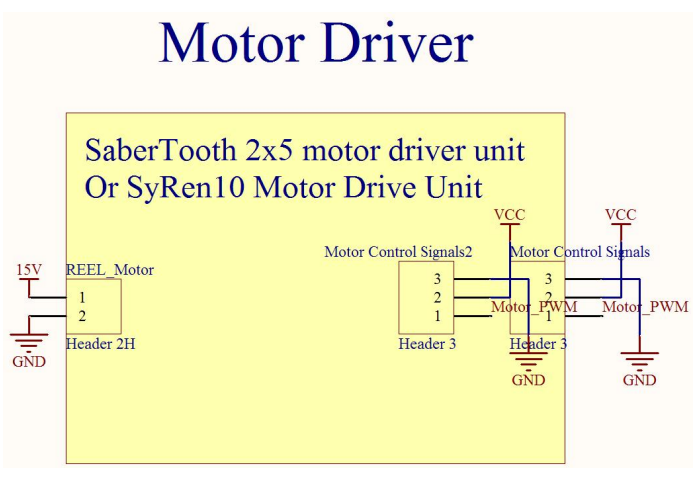

(a)
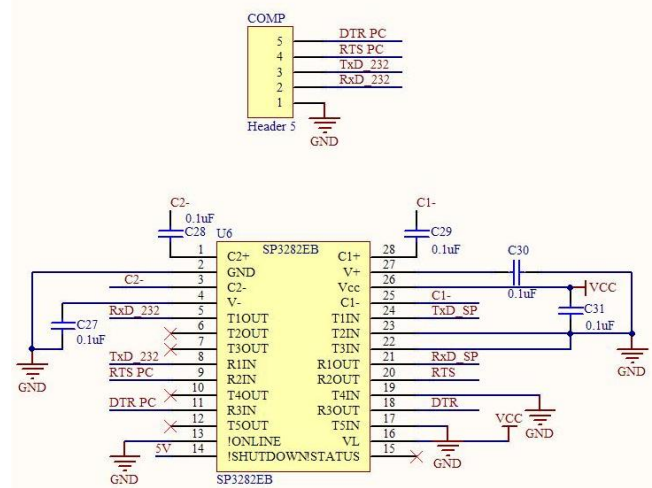

(b)

\section{I/O Headers}

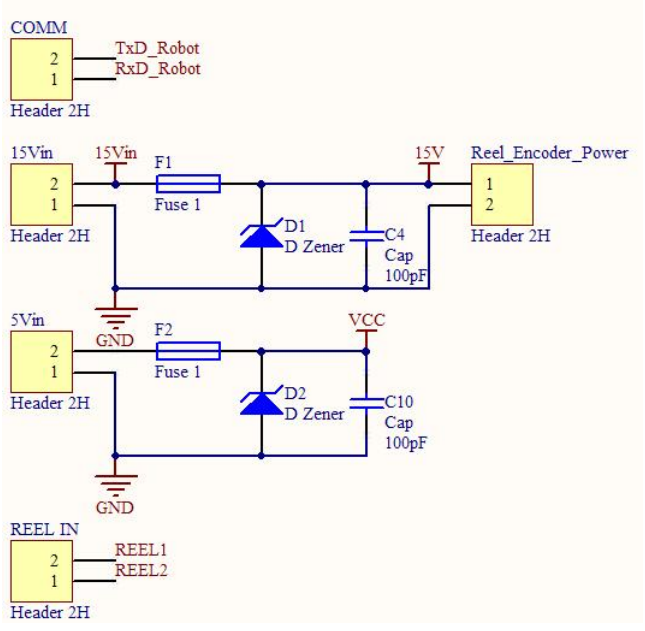

(c)

\section{Diagnostic Port}

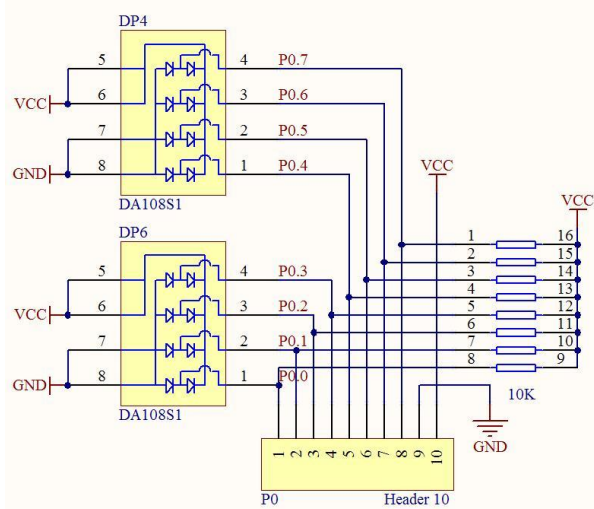

(d)

Figure 5.2: Schematics of Base Station Board revision 1, (a) Motor driver inputs for either Sabertooth $2 \times 5$ or SyRen10; (b) Chip required to reprogram microcontroller; (c) Inputs and outputs with over voltage and noise protection; and (d) Diagnostic port added for testing. 
The PCB (Figure 5.3) is designed so that either a Sabertooth $2 \times 5$ or SyRen10 motor driver (section 6.2.3) can be directly mounted onto the board to reduce the space taken up by the circuitry and to make the connection more reliable.

A testing port (Figure 5.2(d)) is included on this board so that values can be output onto an I/O module so that the data can be checked during the testing phase. This board gives the ability to test and characterise the functioning of the base station.

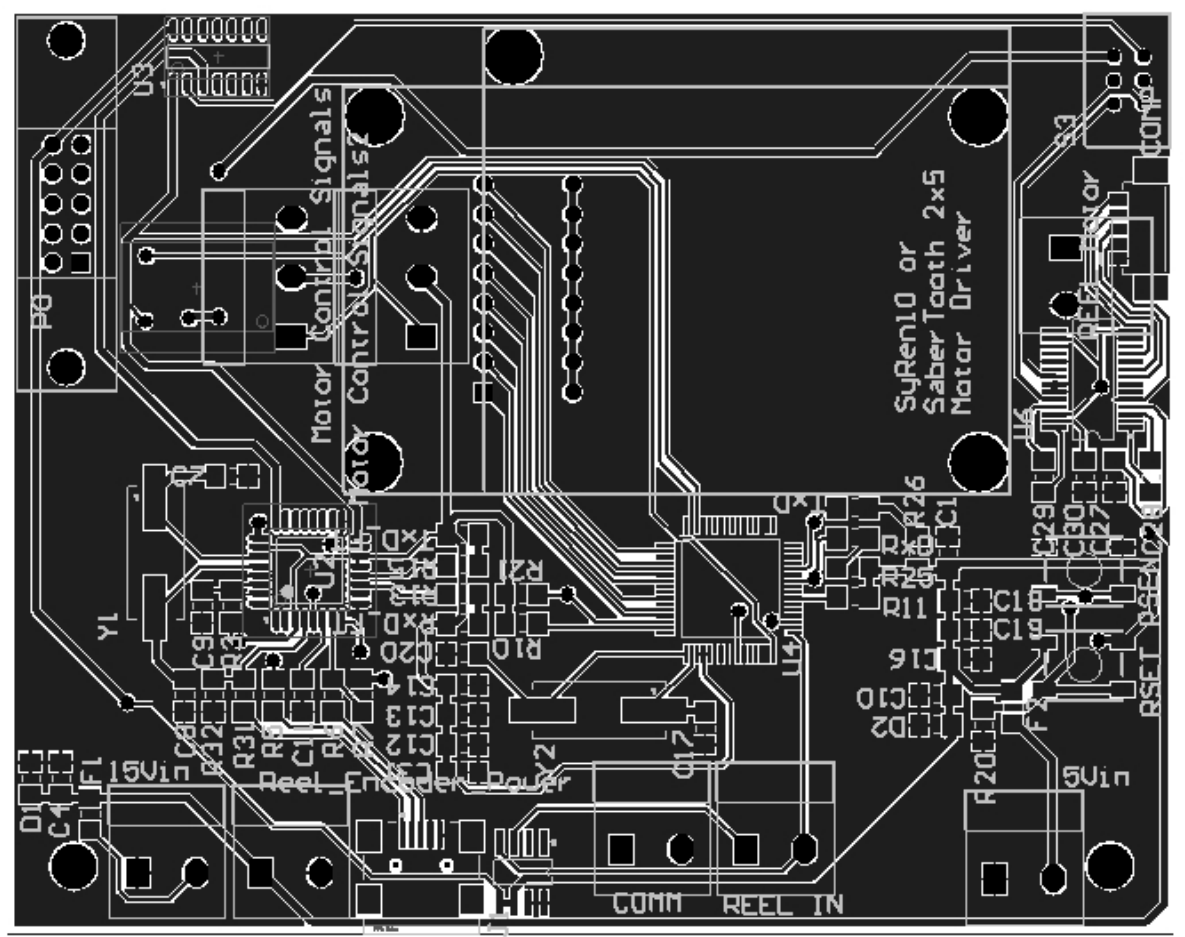

Figure 5.3: Top layer of PCB layout of Base Station Board revision 1

\subsubsection{Revision 2}

In the second revision, the I/O port has been removed as all ports and data that might need to be tested can easily be reached without this port. The mounting for a Sabertooth $2 \times 5$ or a SyRen10 motor drive has also been removed as the estimated motor required to control the reel draws too much current for either of these motor drivers to handle. This was unknown at the time of first revision as the reel supplied for this project by AES was stated as a motorised reel with 

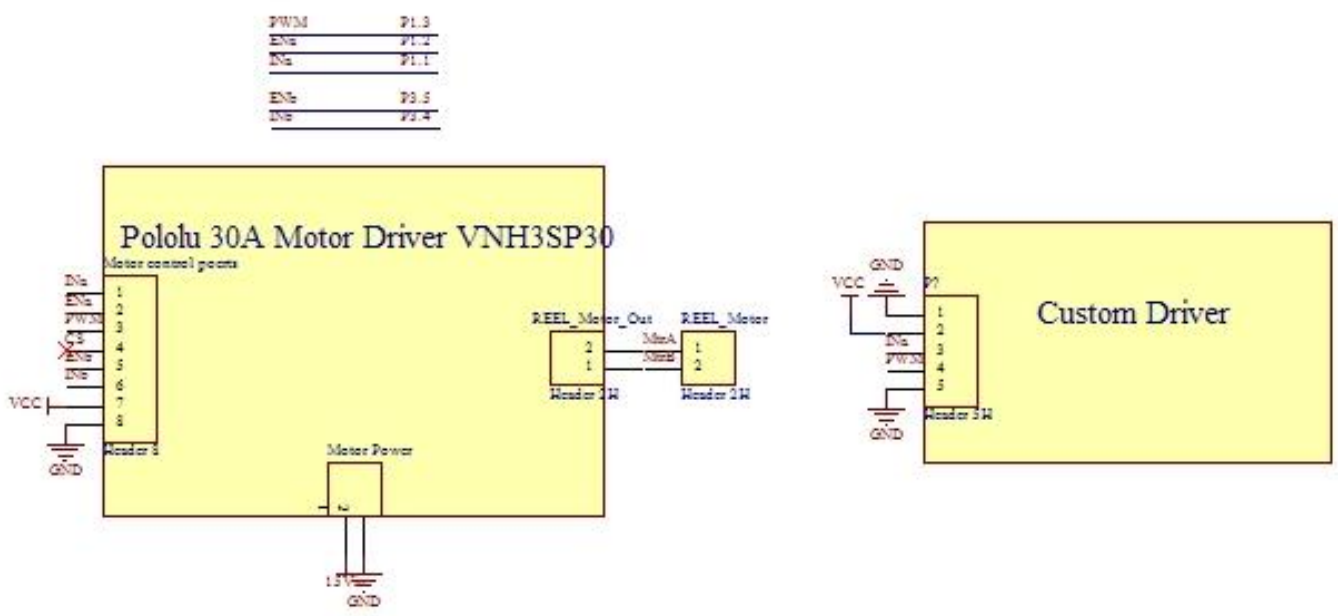

Figure 5.4: Motor driver addition to schematic of Base Station Board in revision 2.

and inbuilt motor. This was found to be incorrect and the device attached to the reel (Lition AC-4898-12) is a slip ring arrangement and not a $12 \mathrm{~V}$ motor. Because of this, the interfacing of a suitable motor driver is needed.

The first option is a Pololu VNH3SP30 30 A motor driver suitable for a reel motor rated at under $30 \mathrm{~A}$ maximum drawn current. The second option is a custom made motor driver (section 5.2) more suitable for motors that draw up to 130 A (Figure 5.4). Both options have been interfaced onto the board (Figure 5.5). AES would prefer the commercially available motor driver as it is the easiest to replace. Unfortunately the most applicable motor to run the reel with the possibility of a free running state is a winch motor. Some of the small versions of these draw up to $80 \mathrm{~A}$. A custom motor driver has been designed due to suitable commercial motor drivers costing a minimum of USD\$219 [31].

Additionally a phono video connector has been included on the board to allow for easy access to the video signal from the robot. With this, a standard RCA cable can be connected between the board and the video capture device attached to the computer. 


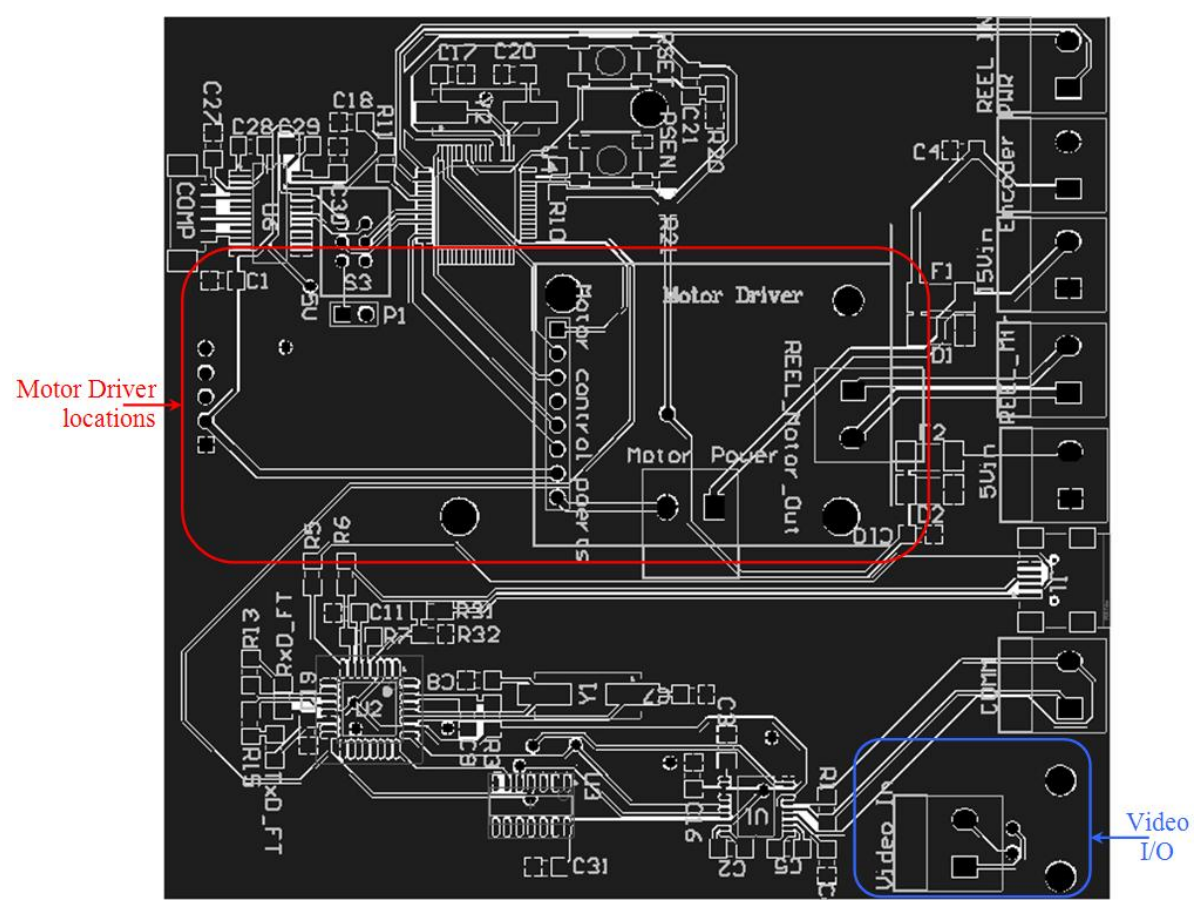

Figure 5.5: Motor driver additions of motor driver (red) and video connection (blue) in PCB layout.

\subsubsection{Revision 3}

The final modification to the base station board was to remove the programming circuitry (Figure 5.2(c)) from the board (this has also been done on the main control board in section 4.2.4). With the use of a 10 pin header an external programming board can be connected to the base station or main control board for reprogramming of the microcontroller. This circuitry is not required for controlling of the robot so has been replaced with a 10 pin header (Figure 5.6) that is identical to that on revision 4 of the main control board. The programming board design is explained in detail in section 5.4.

This revision is currently incomplete and will be modified significantly before manufacturing. The modifications required are to setup the correct type of motor driver which cannot be done until the motor and driver required have been determined. 


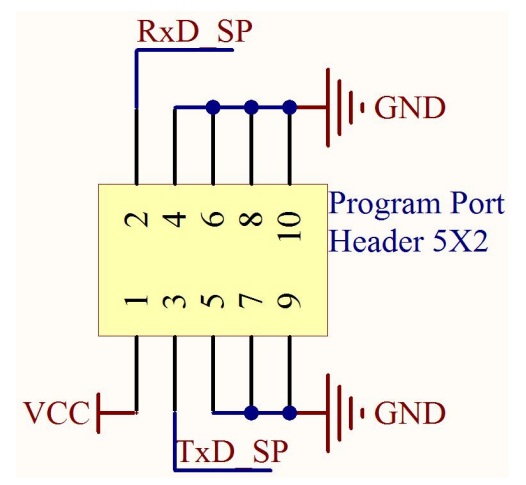

Figure 5.6: Modification to base station board in revision 3 which involves replacing circuitry shown in Figure 5.2(c) with this port to interface with a separate programming board.

\subsection{Motor Driver Board}

The motor driver board is a custom made motor driver designed to control the motorised reel. The requirements for this reel motor are presented in section 6.2.7 since the current chapter discusses PCB design only. This board has been designed to slot directly onto the base station board and accommodate a wide range of motors. This is especially useful since the design of the reel motor was outside the original brief of this project and so voltage and current requirements were unknown. Indicatively commercial winch motors running off $12-330 \mathrm{~V}$ can draw up to $180 \mathrm{~A}$.

The motor driver design incorporates a full H-Bridge utilising IGBTs (Insulated Gate Bipolar Transistors) and a logic driver IC chip. This driver is designed for high current handling capability and hence multiple connections are needed for the H-Bridge supply and motor output to handle the required current. A 4-way screw terminal was used for each of the inputs and outputs allowing the driver to handle up to $110 \mathrm{~A}$ of throughput at up to $300 \mathrm{~V}$. The maximum current and voltage is then limited by the IGBTs used in the configuration. The motor chosen would preferably be a $12 \mathrm{~V}$ motor as this can be supplied by the base station transformer without additional conversion. 


\subsubsection{Revision 1}

Only one revision of the motor driver has been made since at the completion of this project a suitable motor had not been sourced by AES and hence testing was not possible. Four IXTQ130N10T IGBTs are used which are able to supply up to 130 A to a $100 \mathrm{~V}$ motor. The IGBTs are attached to heat-sinks to prevent them from overheating using a custom footprint which incorporates the pinout of the IGBT, the legs of the heat-sink and the overall size of the combined package. Keep-out layers are added to all sides to prevent components from overlapping and shaped pads were added for the legs of the IGBT and the heat-sink (Figure 5.7).

A TC4469CPD Logic-Input CMOS Quad Driver is used to provide the driving circuitry for the motor driver. This chip takes a PWM signal and a direction input and outputs four control signals to the IGBTs. Slight modifications have been made to the circuit diagram given by the manufacturer (Figure 5.8). These involve running the driver IC from a separate power supply than the motor. This prevents the IC from being destroyed by any voltage spikes caused by emf from the motor and to allow the motor to run at a much higher voltage as the driver chip is no longer a limiting device. When the H-bridge and driver IC are run off the same supply, the IC has the lower voltage rating, limiting the maximum voltage that is able to be supplied to the circuit and therefore,

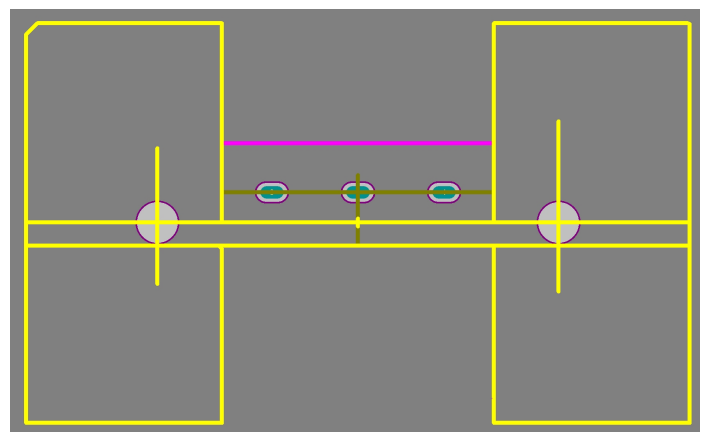

Figure 5.7: Custom made footprint for IGBT and heatsink combination 


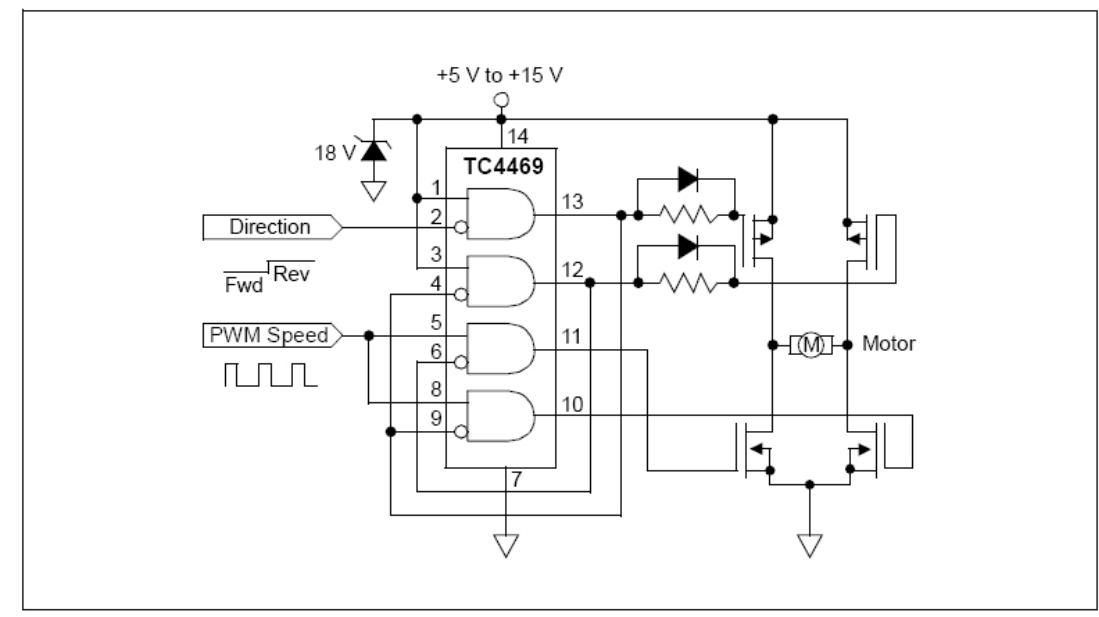

Figure 5.8: Connection Diagram of TC4469CPD driver IC

the motor. Hence the driver IC is powered from a $5 \mathrm{~V}$ supply line whereas the H-Bridge is powered by a $15 \mathrm{~V}$ supply line (Figure 5.9(a)).

The overall design of the motor driver is such that it is compact as well as being easily serviceable should a component fail(Figure 5.9(b)). The driver IC chip is a DIP (Dual In-Line Package) which fits directly into a socket, hence if the driver fails a new one can be inserted quickly and easily. The IGBTs are screwed directly to separate heat-sinks which are not soldered to the board but are firmly held in place by the IGBTs. This has been tested by inserting the heat-sink and IGBT package into the board, inverting the board and shaking.

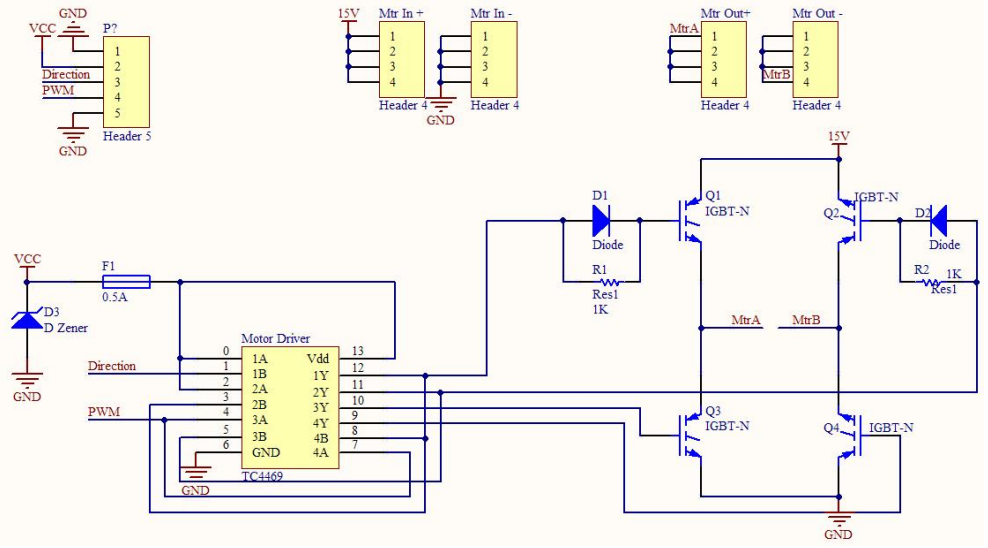

(a)

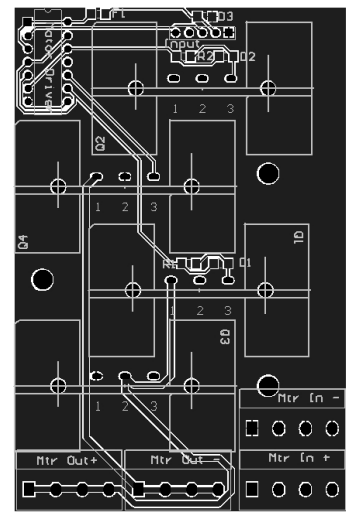

(b)

Figure 5.9: Reel Motor Driver Revision 1 (a) schematic and (b) top layer of PCB showing the location of the IGBTs with heatsinks and other components. 
The packages held to the board without the help of solder. The IGBTs are soldered to the board on the bottom side only. Due to the small size of the pads this solder can easily be removed if the IGBTs need to be replaced. The zener diode and fuse used as protection components for the driver IC have been placed on the outer edge of the board design so that they can easily be replaced if they malfunction.

All input and output plugs have also been placed on the outside of the board, the motor I/O ports have all been placed on the same side so that it is possible to house the motor driver in a box and have all wires coming from one side of the board. When this board is mounted onto the base station (section 5.1) the ports are on the same side as the base station ports facilitating easy interconnection.

\subsection{Camera Test Board}

The camera test board is designed to test the full functionality of the newly acquired Sony FCB-H11 camera module and discover the most applicable interfacing option for this project. This board contains 2 ports, one for the FFC (Flat Flexible Cable) that connects to the camera module and one DIP format single line header that wires can be connected to so that the functions of the camera can be tested (Figure 5.10(a)). The PCB layout of this board (Figure $5.10(b))$ has the function names of each pin written into the copper pour of the board as well as the top overlay. This is due to the PCB manufacturer supplying two copies of each board, one of which does not include a top overlay. Thus including the names in the copper pour allows for use of the board without referral back to the layout. 


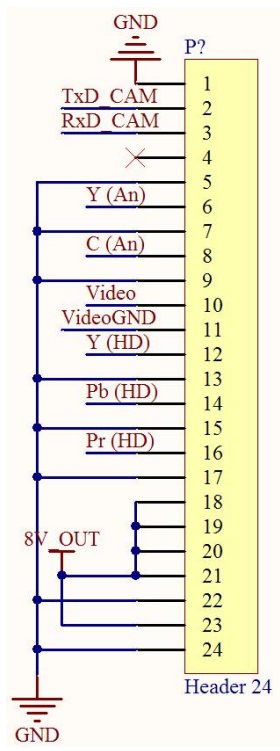

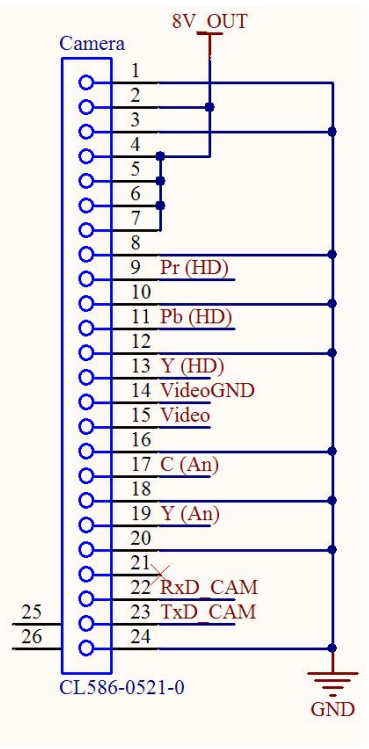

(a)

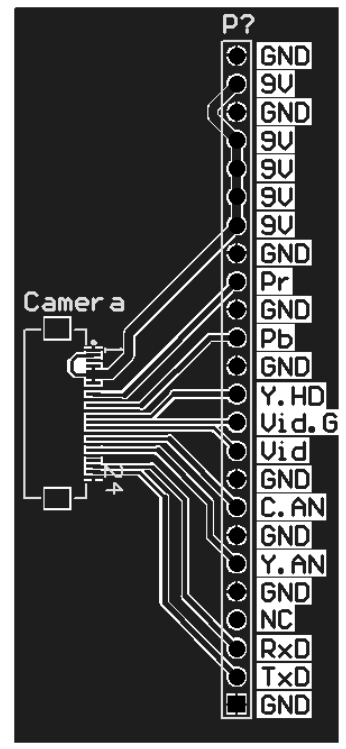

(b)

Figure 5.10: Sony FCB-H11 Camera Test Board (a) schematic and (b) PCB layout

\subsection{USB to Serial Board}

A USB to serial conversion board is designed to convert the USB signal to the serial communication required for programming of the microcontrollers.

Due to problems that arose when using a USB to serial communication board designed by electronics technician Jason Edwards, a USB to serial board was designed specifically for this project. The additions to this board have been made to protect the components from damage by over voltages on any of the lines. These additions include a diode pack which protects the FT232 chip from spikes that may be returned from the microcontroller and a zener diode between the $5 \mathrm{~V}$ and ground lines to prevent an over voltage on the power supply line.

The board contains a mini USB input plug which is used to connect the computer to the board. The board is connected to either the base station or the main control board via a 10 pin header which provides the transmit and receive lines as well as the positive and negative power supply lines. 


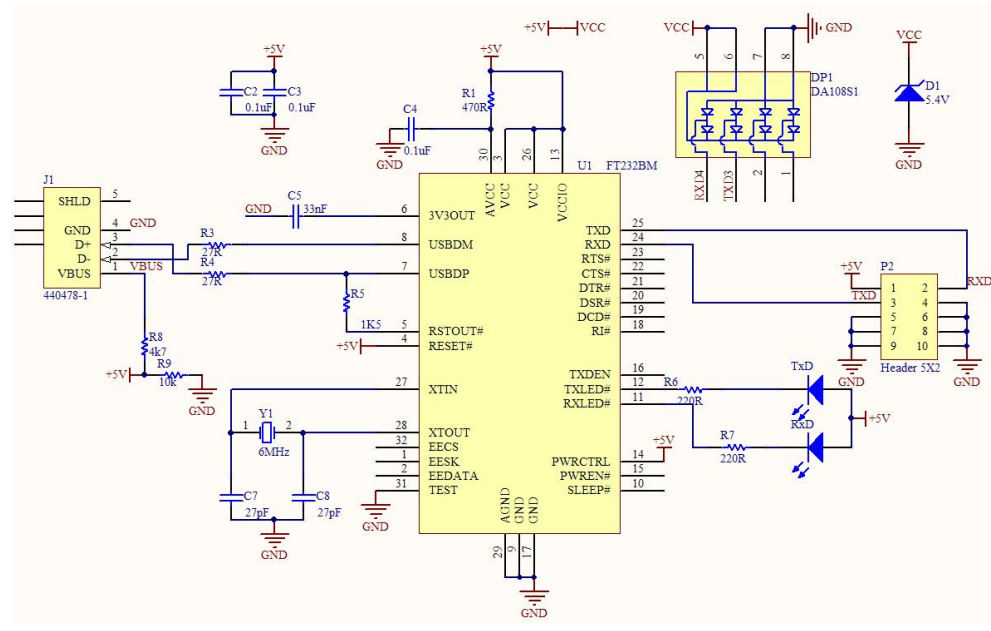

(a)

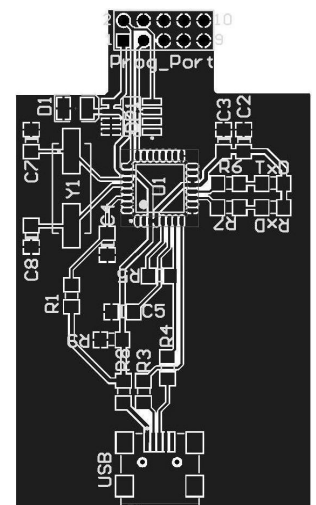

(b)

Figure 5.11: USB to serial communication board (a) schematic and (b) PCB layout.

To implement the USB to serial RS-232 transition a FT232 chip is utilised and is illustrated in Figure 5.11(a). The shape of the board design is also important as the 10 pin header must be able to connect to its reciprocal on each of the other boards. The resulting design is displayed in Figure 5.11(b). If the 10 pin header was not extruded away from the bulk of the board it would not be possible to connect to the main control board as the two screw terminals bracketing the reciprocal plug would cause the mating to be impossible. This board was designed at same time as third revision of the main control board section 4.2.3.

\subsection{External PCB Summary}

A USB to serial board has been designed for programming of the main control and base station boards. This board reduces the number of components on both the main control and base station boards making debugging and replacement easier.

A camera test board has also been created to test the functionality of the newly acquired Sony FCB-H11 camera module (section 6.2.1). 
The base station board allows for connection to a commercial and custom motor driver as well as providing all serial and video communication between the computer and robot.

The current version of the motor driver board is able to control $100 \mathrm{~V}$ motors drawing $110 \mathrm{~A}$, a modification to controlling a higher voltage or AC motor might be required at a later stage. The control pins from the base station board provide both a PWM signal and directional control signal if needed. 



\section{Chapter 6}

\section{Lights, Camera, Action}

This chapter outlines the hardware devices used in the project, including the Sony camera block modules and the internal motor drivers.

The chapter is divided into two sections, custom made and commercially available hardware devices including the devices used in this project and the process of determining which to use at later stages in the system development.

\subsection{Lights}

The lights used for illuminating the inside of the pipe need to be bright and preferably a white light so that the colours of the video feed are consistent with the true colours inside the pipe. Ideally the lights would use a low amount of power to reduce the internal heating of the robot. Ristic acquired two Weldon Technologies high power, high brightness LEDs (Figure 6.1) via Farnell for testing. On these LEDs he soldered two wires so that they could be plugged into the head of the robot.

Replacement of these LEDs required a significant amount of time as the mounting of these LEDs into the head of the robot required blindly inserting two 


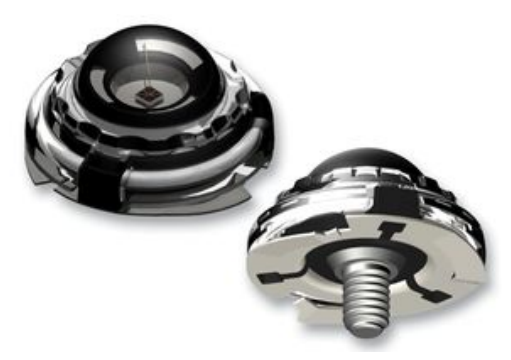

Figure 6.1: Weldon Technologies $110^{\circ} 80 \mathrm{~lm}$ white LED with ground base plate and positive screw terminal.

wires into sockets. Hence an objective in the redesigning of this system is to devise a means for quick and easy replacement of the LEDs whilst maintaining water-tight integrity. This is accomplished by designing a housing for the LED that plugs into the sockets of the robotic head. The housing is divided into seven pieces, five of which are shown in Figure 6.2, the other two components are rubber o-rings to provide the water-tight sealing. The back end of the housing (housing end in Figure 6.3) is screwed into the robotic head with an o-ring in-between to provide sealing between the components. The base plate and screw-in pin are combined with the plastic insert in-between to separate the positive and negative sides of the LED. This is then inserted into the housing end as shown in Figure 6.3. The cover is screwed on to the top of the housing end once an LED has been screwed into the screw-in pin. The final o-ring is inserted between the LED and the cover creating a water-tight seal throughout the components. This was designed in SolidWorks and built by Manu Pouajen-Blakiston at the university's mechanical workshop. The com-

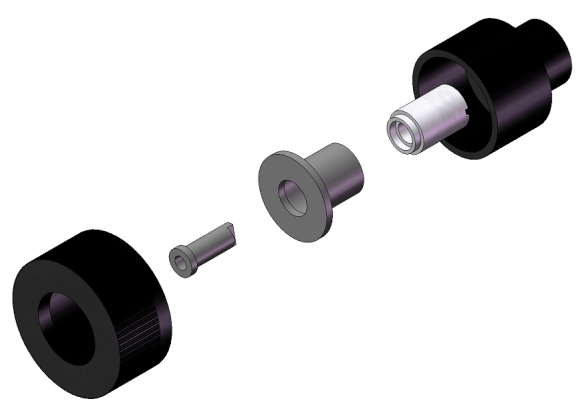

Figure 6.2: LED socket designed specifically for quick replacement of Weldon Technologies screw terminal LEDs whilst maintaining water-tight sealing. 


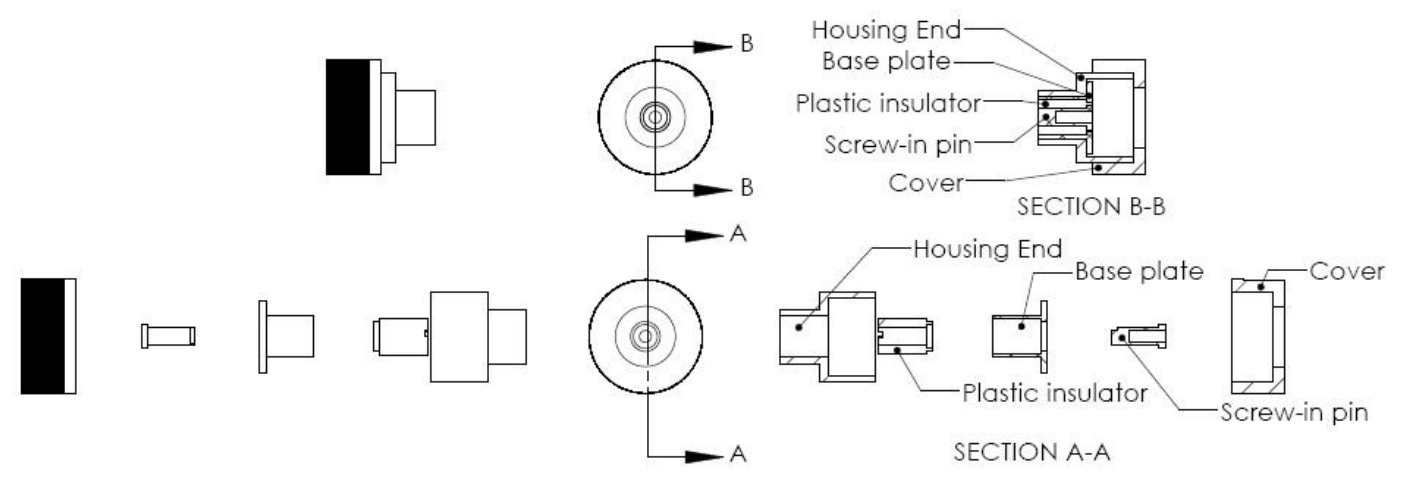

Figure 6.3: LED socket drawing showing the holder collapsed and exploded thus displaying how components fit together.

pleted housing now allows for easy fitting and replacement of the LEDs.

\subsection{Commercial peripherals}

\subsubsection{Cameras}

In this project two cameras are considered, the first being the Sony EVI-371 camera block module (Figure 6.4(a)) that was originally supplied with the robot. This camera is a compact CCD (charge coupled device) camera block with $12 \times$ optical zoom, $10 \times$ digital zoom, and an auto focus lens assembly. It utilises a 1/3 inch Hyper HAD (hole-accumulation diode) CCD capture array with 440,000 effective pixels and is able to be used in PAL operation mode resulting in a resolution of $460 \mathrm{TV}$ lines. This series of camera uses the VISCA ${ }^{T M}$ interface protocols (section 6.2.2) which allows for digital control of all camera functions. This camera has horizontal and vertical view angles of $48.8^{\circ} \times 37.6^{\circ}$ when operated in wide mode and view angles of $4.3^{\circ} \times 3.2^{\circ}$ when operated in telescopic mode, accomplished by adjusting the 9 elements in the camera lens assembly to vary the focal length between $5.4 \mathrm{~mm}$ and $64.8 \mathrm{~mm}$.

The camera is a low power device drawing under $4 \mathrm{~W}$ continuously and is able to operate on voltages between $6 \mathrm{~V}$ and $12 \mathrm{~V}$. It also has the option of 


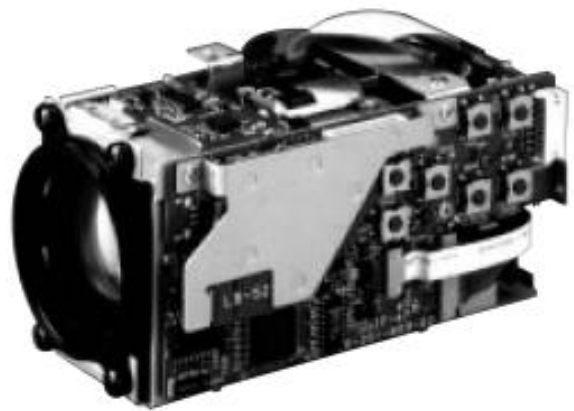

(a)

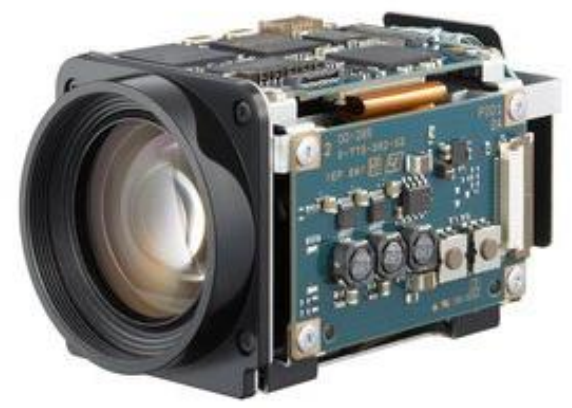

(b)

Figure 6.4: Sony (a) EVI-370 and (b) FCB-H11 camera modules

outputting video in the form of YC format or VBS format, of which VBS is used in the original system due to the signal coaxial cable requirement (section 6.2.6). Weighing $225 \mathrm{~g}$ with dimensions of $54 \mathrm{~mm} \times 50.8 \mathrm{~mm} \times 100.1 \mathrm{~mm}$ (width $\times$ height $\times$ depth) this camera still competes with cameras used in current pipe inspection systems despite being over 10 years old!

The second camera considered is the Sony FCB-H11 camera block module (Figure 6.4(b)) which has been donated to this project by Geremy Brown, product manager of Sony New Zealand Limited. This camera retails for USD\$1,260 [32] and is a two megapixel full high definition capable, compact camera module, using a 1/3 inch HD CMOS (complimentary metal oxide semiconductor) sensor. The lens of the camera has a $10 \times$ optical zoom and the optional function of $12 \times$ digital zoom, this allows for in-depth analysis of the region. This compact camera has the dimensions of $47.2 \times 43.1 \times 72.2 \mathrm{~mm}(\mathrm{w} \times \mathrm{h} \times \mathrm{d})$ and weighs 120 g. Additionally, the camera is connected via one 24 pin Flat Flexible Cable (FFC) which provides all control and power inputs as well as all video outputs to and from the camera.

This camera contains both standard analogue video outputs (component and composite video) and HD analogue video in the form of a component video output. The essential difference between the component and composite types of video output is the amount of data sent down the lines. The composite 
video usually consists of a single coaxial connector which sends a "Colour, Video, Blank and Sync" data packet. Component video usually consists of 3 coaxial connectors for video in the form of RGB, YPbPr or YCbCr (section 3.2.4) allowing for greater colour depth and video resolution.

The 2 MP resolution corresponds to an output of 1224 TVLs in the standard 4:3 television aspect ratio (equation 6.2), this is significantly better than the 460 TVLs produced by the Sony EVI-371.

$$
\begin{aligned}
\text { aspectratio } & =4 n: 3 n \\
4 n \cdot 3 n & =\text { screensize }
\end{aligned}
$$

hence

$$
12 n^{2}=\text { Pixels }
$$

therefore

$$
\text { ratio }=4 \cdot(\sqrt{\text { Pixels } / 12}): 3 \cdot(\sqrt{\text { Pixels } / 12})
$$

As the camera is a Sony module, the setup of the non HD component video uses the SoG format. The SoG setup is to send a sync signal down on the green line of an RGB signal. Extra circuitry is required to remove the synchronisation before displaying the video otherwise a green tinge can appear. Computer monitors are not designed to run SoG hence an adaptor is required if a computer is used for video display. Sony is one of the only manufacturers to do this hence using one of their modules with incompatible acquisition devices can result in a green tinge to the image. The other option for using the component cable setup is to use HD video which adheres to the High-Definition Multimedia Interface (HDMI) standards. Although this setup will provide significantly better video due to the increased colour depth and higher resolution, one disadvantage is that a HD video acquisition device is required. A USB version has been found, although it requires a portion of the computer's processing power to run as will be explained in section 7.1.4. 


\subsubsection{Camera Functions}

Both the Sony EVI-371 and Sony FCB-H11 colour camera modules have similar hardware profiles and functions. Both of these cameras are classed as auto focusing cameras with the possibility of manual adjustment, also both have a maximum zoom of $120 \times$. Their functions can be controlled via serial data packets (see section 3.1.2). This data packet causes a hardware manipulation inside the camera. The instruction Table 6.1 shows the required data packet transmission for a desired manipulation. This Table is given in the datasheet for this camera series, locatable on the appendix CD.

Page 10 of the Sony FCB-H11 technician's manual (see Appendix CD) and shows that the VISCA ${ }^{T M}$ commands for the Sony FCB-H11 camera are mostly the same as those for the older EVI-371 camera. One difference however, is that the newer camera has additional functions including an array of inquisition functions and does not have the ability to set or display the current time and date of the camera. This is a slight hindrance as the time and date of the inspection is a requirement of AES cliental. To overcome this a time and date stamp will needed to be added to the video stream by the recording device or software at a later stage.

Table 6.1: Relevant section of the Sony EVI 370 series camera command list.

\begin{tabular}{lll}
\hline Command Set & Command & VISCA $^{T M}$ Packet \\
\hline CAM_Power & On & $8 \times 01040002 \mathrm{FF}$ \\
& Off & $8 \times 01040003 \mathrm{FF}$ \\
CAM_Zoom & Stop & $8 \times 01040700 \mathrm{FF}$ \\
& Tele (Standard) & $8 \times 01040702 \mathrm{FF}$ \\
& Wide (Standard) & $8 \times 01040703 \mathrm{FF}$ \\
CAM_Focus & Stop & $8 \times 01040800 \mathrm{FF}$ \\
& Far & $8 \times 01040802 \mathrm{FF}$ \\
& Near & $8 \times 01040803 \mathrm{FF}$ \\
CAM_DateDisplay & On & $8 \times 01072 \mathrm{a} 02 \mathrm{FF}$ \\
& Off & $8 \times 01072 \mathrm{a} 03 \mathrm{FF}$ \\
CAM_TimeDisplay & On & $8 \times 01072 \mathrm{~b} 02 \mathrm{FF}$ \\
& Off & $8 \times 01072 \mathrm{~b} 03 \mathrm{FF}$
\end{tabular}




\subsubsection{Motor Drivers}

The Sabertooth $2 \times 5$ motor driver is a commercially available dual motor driver unit able to provide $5 \mathrm{~A}$ to two motors running at up to $18 \mathrm{~V}$. As this is a dual driver the space required for the motors and control is significantly reduced (compared to installing 2 discrete drivers) as well as making replacement of parts more simplistic.

Figure 6.5(a) shows the setup requirements for the Sabertooth $2 \times 5$ motor driver which requires a battery input, a control input for each motor, a common ground input and two dual connections to the motors. This driver has been mounted to the inside of the robot lid and wired directly to the main control board (Chapter 4.2). It is used to control both camera head motors. To configure the motor driver into the required mode it has a 6 switch DIP switch block. For this control system, the DIP switches are arranged to set the motor driver into independent analogue mode, which allows the control of each motor from a separate analogue input signal. The DIP switch arrangement required is found using either the motor driver datasheet or the DIP switch configuration wizard available on the Dimension Engineering website[33].

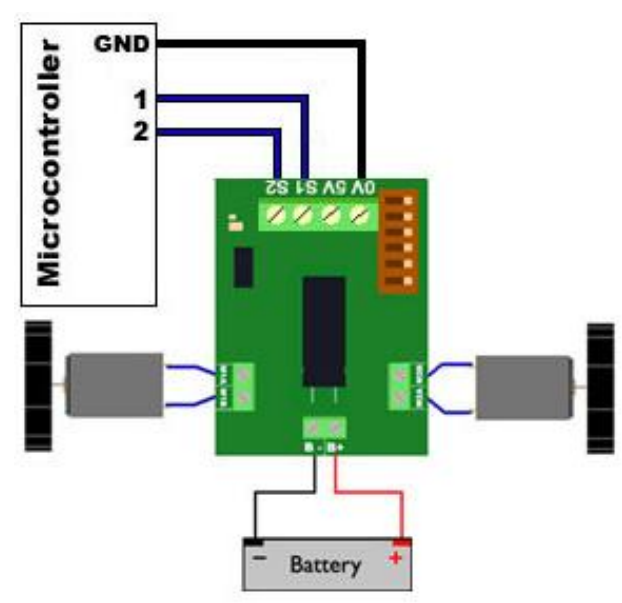

(a)

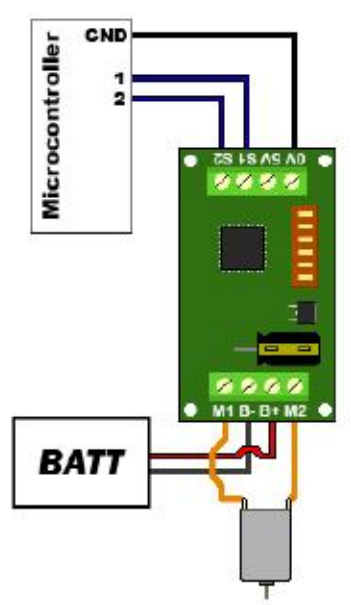

(b)

Figure 6.5: (a) Sabertooth 2×5 motor driver setup, (b) SyRen10 motor driver setup 
The other motor driver used inside the robot is a SyRen10, 24 V 10 A single motor driver which is able to run at a maximum of $30 \mathrm{~V}$. This is used to control the $24 \mathrm{~V}$ drive motor of the robot. The motor driver is setup in bidirectional $5 \mathrm{Vpp}$ analogue input mode where a $2.5 \mathrm{~V}$ input will stop the robot, an input under $2.5 \mathrm{~V}$ will cause the robot to reverse at increasing speed and an input over $2.5 \mathrm{~V}$ will cause the robot to move forward with increasing speed. Figure 6.6 shows the relationship between the input and the robot's movement. Note the dead zone around the stop value as the motor must overcome any static friction before movement can occur. The motor driver is wired to the $24 \mathrm{~V}$ supply from the Vicor DC-DC converter (section 4.4), a control and ground input from the main control board and provides a dual output to the motor (Figure 6.5(b)). A second control signal has been connected to the motor driver in case greater sensitivity is required. This allows the use of the direction control and speed control option of the motor driver by adjusting the DIP switches and the microcontroller code. In this configuration, one 0 to $5 \mathrm{~V}$ input controls the

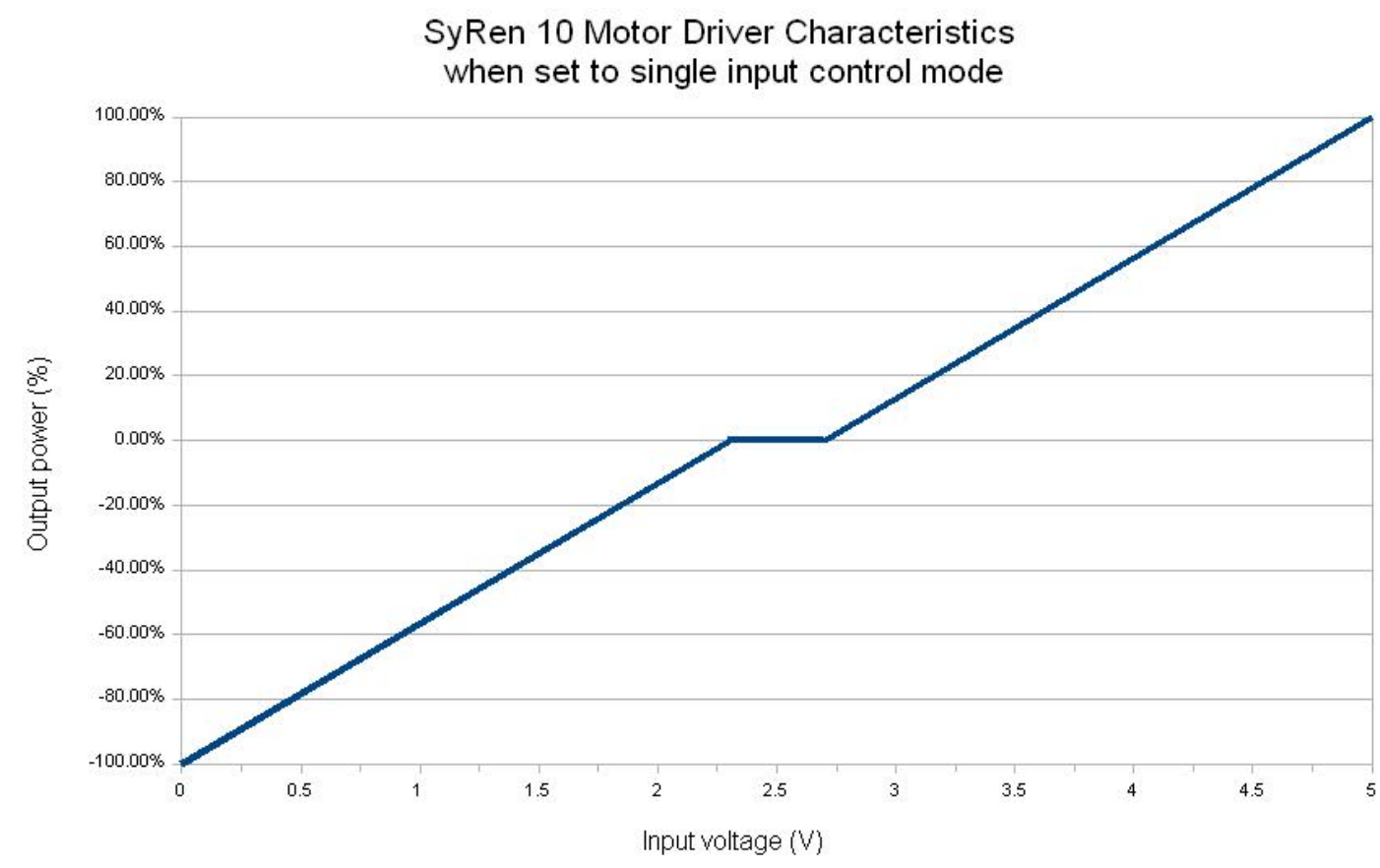

Figure 6.6: Characteristics of SyRen 10 Motor Driver used to control tractor drive motor. Where the output is a percentage of the maximum possible power of the $24 \mathrm{~V}$ motor connected to the driver. Actual dead zone size varies slightly between the motors. 
speed of the motor and the other controls the direction.

Table 6.2: Sabertooth $2 \times 5$ and SyRen 10 motor drive DIP configurations used to control the 3 internal motors with switch functionality explained.

\begin{tabular}{|c|c|c|c|}
\hline Pin & Function & $\begin{array}{l}\text { Sabertooth } \\
2 \times 5\end{array}$ & SyRen \\
\hline 1 & $\begin{array}{l}\text { Mode selection switch } 1 \text {, the modes that the motor } \\
\text { drivers can use are selected using switches } 1 \text { and } 2 \text {. } \\
\text { These modes are Analogue Input, R/C Input, Sim- } \\
\text { plified Serial and Packet Serial. For switch config- } \\
\text { urations see Sabertooth or SyRen datasheets on the } \\
\text { appendix CD. }\end{array}$ & UP & UP \\
\hline 2 & Mode selection switch 2 , see switch 1 for details. & UP & UP \\
\hline 3 & $\begin{array}{l}\text { Lithium cut off switch. This is only set DOWN if } \\
\text { lithium batteries are used as the motor driver is able } \\
\text { to detect the number of cells and prevent under and } \\
\text { over voltages damaging the batteries. }\end{array}$ & UP & UP \\
\hline 4 & $\begin{array}{l}\text { Sabertooth: Mixed or independent mode selection } \\
\text { if switch is UP the driver is useful for differential } \\
\text { drive vehicles. When DOWN each signal controls a } \\
\text { singular motor. } \\
\text { SyRen: Bi-directional control switch. If UP a } 0 \\
\text { to } 5 \mathrm{~V} \text { input controls the motor in both directions } \\
\text { where } 2.5 \mathrm{~V} \text { stops the motor, if down the motor op- } \\
\text { erates only in forward direction. }\end{array}$ & DOWN & UP \\
\hline 5 & $\begin{array}{l}\text { Sabertooth: Exponential response to input if switch } \\
\text { is DOWN, linear if UP. } \\
\text { SyRen: secondary control switch. If DOWN signal } \\
2 \text { is used to control direction of motor while signal } \\
1 \text { controls speed of motor. }\end{array}$ & UP & UP \\
\hline 6 & $\begin{array}{l}\text { Sabertooth: input range is between } 0 \mathrm{~V} \text { and } 5 \mathrm{~V} \\
\text { if switch is UP. Driver is in } 4 \times \text { mode if switch is } \\
\text { DOWN, operating between } 1.875 \mathrm{~V} \text { and } 3.125 \mathrm{~V} \text {. } \\
\text { SyRen: Reverse switch. If DOWN motor output in- } \\
\text { verted compared to driver input. }\end{array}$ & UP & UP \\
\hline
\end{tabular}

The configurations displayed in Table 6.2 are those used to control the three motors in an independent fashion as well as explaining the operation of each pin. Although the motor drivers have the ability to be controlled by a serial input, the analogue mode is used as the microcontrollers serial communication line is in use (communication with the computer via the base station). Additionally analogue mode is easy to implement with the use of a PWM signal. 


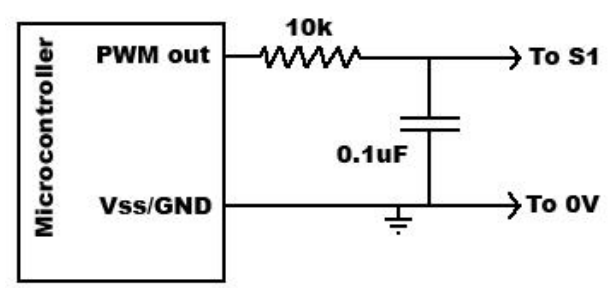

Figure 6.7: Motor driver low pass filter required to convert PWM output of microcontroller to an analogue input signal for the motor drivers.

As the microcontroller on the main control board (Chapter 4.2) is a digital device outputting a digital PWM signal at approximately $12 \mathrm{kHz}$, an analogue approximation of the signal needs to be implemented so that the motor is able to be controlled in a smooth fashion. The filter network required to do this is a basic RC low pass filter with a cutoff frequency of $1 \mathrm{kHz}$ (Figure 6.7).

\subsubsection{Joystick}

The GUI designed for this project involves the use of a joystick to allow for intuitive control of the robot (section 7.1.2). The joystick chosen is a Logitech Extreme $^{T M}$ 3D Pro gaming joystick (Figure 6.8). It has 12 buttons that can be programmed for different functions of the robot (an explanation of the programmed functions can be found in section 7.1). The joystick is also a three

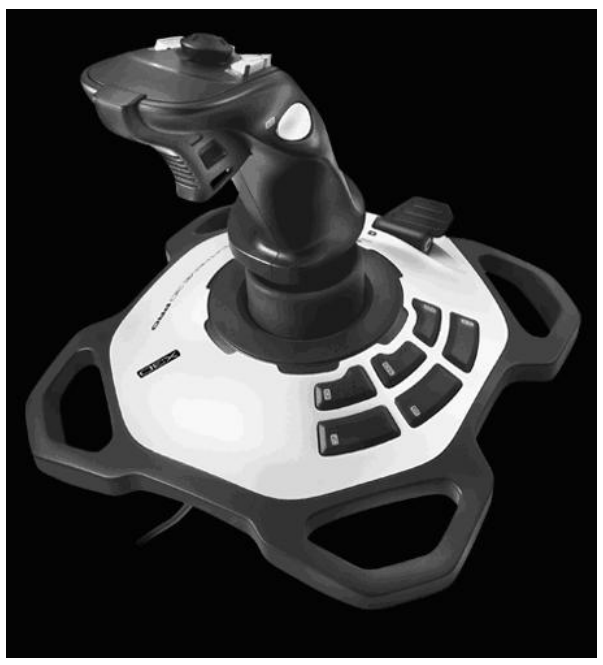

Figure 6.8: Logitech Extreme 3D Pro joystick used for control of the robot. 
axis joystick with a comfortable yoke design and stable base plate, which is useful as the technicians must complete runs that maybe up to 1 hour long hence a comfortable control system is necessary. The three axes of movement allow for the three degrees of movement present in the robot, these are forward and backward movement of the unit itself, and rotation and panning of the camera head. Each axis provides a 16 bit input to the computer which is then scaled to the desired output in the GUI.

\subsubsection{Internal Motors}

During initial testing to determine the power requirements of the system it was found that one of the camera motors was broken, specifically the camera rotation motor. The back end of this motor where the wires connect to it was missing one of the connectors, hence the camera would not rotate without the wire being manually held in place.

Thus a motor that would be able to provide similar specifications as the one currently attached to the system needed to be found.

The motor attached to the system was determined to be a Faulhaber 2225U012S motor which is a $12 \mathrm{~V}$ micro motor with a diameter of $22 \mathrm{~mm}$ and length of $25 \mathrm{~mm}$. This is connected to a 592:1 planetary gearbox (Faulhaber 23/1 series) before being connected to the camera gear train described in section 2.2. The standard electronic suppliers for the university (Farnell[25], RS[34], JayCar[35], Mouser[36] and Digikey[30]) were checked to see if they were able to provide a motor with the correct specifications. None were found to be suitable and so it was decided to get a replacement Faulhaber motor.

Using the Faulhaber website [37] a suitable motor replacement has been found; the Faulhaber 2224 series motors (Figure 6.9(a)), specifically the $12 \mathrm{~V}$ variant which can supply a continuous speed of 8,000 RPM and a continuous torque 


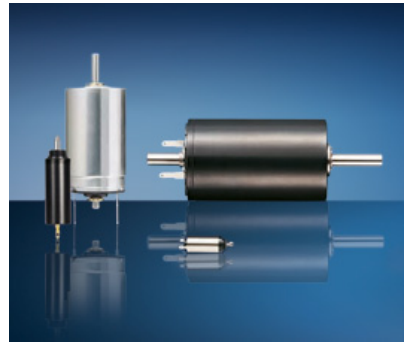

(a)

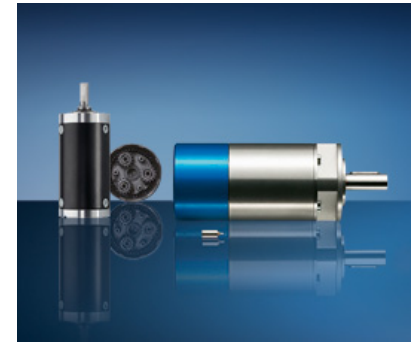

(b)

Figure 6.9: Faulhaber (a) DC-Micromotors, these motors come in an array of sizes from a diameter $\times$ length of $6 \mathrm{~mm} \times 15 \mathrm{~mm}$ to $38 \mathrm{~mm} \times 63 \mathrm{~mm}$ and $(\mathrm{b})$ Planetary Gearboxes, these gearboxes come in sizes from $6 \mathrm{~mm} \times 15 \mathrm{~mm}$ to $44 \mathrm{~mm} \times 214 \mathrm{~mm}$

of $5 \mathrm{mNm}$; it has a stalling torque of $19.8 \mathrm{mNm}$. A replacement gearbox is also available from Faulhaber (Figure 6.9(b)). The maximum efficiency of this 592:1 ratio planetary gearbox is $55 \%$. This was then sourced from the local supplier, Erntec [38].

\subsubsection{Cabling}

The original supplied cable that is towed into the pipes by the robot is in irreparable condition, with the sheath cracked exposing the wires and the cable stretching slightly when pulled. Thus a new cable is required for this robot to become operational. A problem is that all pipe inspection system suppliers have their cables custom made with their name imprinted on the other sheath.

At time of this thesis writeup finalisation of cable choices has not been made due to time constraints, although four manufacturers have been found that might be able to produce suitable cabling.

One is the New Zealand company General Cable [39] which is a manufacturer of multi-core cables, although the outer sheath of the cables might not be able to handle the strain that the robot is possible of putting on the cable.

Falmat[40] (USA) is a custom cable manufacturer that already produces pipe inspection cables. The specifications that they give on their cables are minimal 
and additional information will have to be acquired before a decision can be made.

Lapp[41] and Tyco Electronics [42] are both manufacturers of a large array of cabling options including multi-core and fibre optical cables. These two would likely be able to provide a suitable form of cable.

One specification of the cable that must be met is that the cable is required to handle video transmission which requires a core with a characteristic impedance of $75 \Omega$. In $300 \mathrm{~m}$ video transmission applications RG6 standard coaxial cable is commonly used to limit signal attenuation [43]. The problem with using coaxial cables is that they are bulky, the lightest cable able to be found weighs $26.8 \mathrm{~kg} / \mathrm{km}$ [44] per core assembly. If the full HD capability of the Sony FCB-H11 camera (section 6.2.1) is to be used, three such assemblies would be required inside the cable adding an additional $24 \mathrm{~kg}$ to a $300 \mathrm{~m}$ cable's weight. The overall weight of the cable cannot exceed $50 \mathrm{~kg}$ otherwise the system will no longer be portable. This leaves $26 \mathrm{~kg}$ for $6-8$ other cores as well as cable reinforcement and insulation which might push the boundaries of manufacturing capabilities.

A solution to this that is currently being explored is to use one coaxial cable and one triaxial cable as this should slightly reduce the overall weight and outer diameter of the cable. The outer diameter of the cable is important as it determines how much cable can fit onto the reel. At the present time the cable reel has been calculated to hold up to $250 \mathrm{~m}$ of $8 \mathrm{~mm}$ cable (the diameter of the current cable). The specification of AES is that the robot must travel up to $250 \mathrm{~m}$ down the pipe and it is preferred to have $300 \mathrm{~m}$ of cable on the reel so that excess stress is not put on the equipment. To achieve this, the cable reel must be modified which is a task that will be attempted at a later date after discussion of the possibilities with AES. 
Other specifications for the cable include being able to handle $300 \mathrm{~V}$ at $0.5 \mathrm{~A}$ per core to handle the required power supply and possessing an inert cable sheath due to the sewage pipes containing a large array of chemicals. The sheath must also have minimal to no movement (creep) as the distance the robot has travelled is calculated off the cable. The cable must also be strong enough to pull $100 \mathrm{~kg}$ otherwise it will break when emergency removal of the robot occurs (when the robot breaks down). The recommendation for this is to use a Kevlar reinforced sheath.

The number of rows and windings that the reel can take is determined from equations 6.3 and 6.4 .

$$
\begin{aligned}
\text { Windings } & =\frac{\text { drum_width }}{\text { cable_diameter }} \\
\text { Rows } & =\frac{(\text { reel_diameter }- \text { drum_diameter })}{(2 \times \text { cable_diameter })}
\end{aligned}
$$

Using these equations, the length of cable on the reel can be calculated by determining the length of cable on each row. This length is calculated using the circumference of the winding and the number of windings that are contained on that row (equation 6.5). Expanding this equation for row number $n$ is done by determining the diameter that the cable must wrap around and hence the new circumference. This diameter is calculated by taking the start diameter of the cable drum and adding twice the diameter of the cable for each previous row as the cable loops around the drum. This is then multiplied by the number of preceding rows resulting in equation 6.6. The total length of the cable can then be calculated by simply taking the sum of all rows that will fit on the reel 
(equation 6.7).

$$
\begin{aligned}
\text { length_on_row } & =\text { Windings } \times \text { Diameter } \times \pi \\
\text { length_on_row } & =\text { Windings } \times\left(d_{\text {reel }}+\left(d_{\text {cable }} \times 2\right) \times(n-1)\right) \times \pi \\
\text { Total_length } & =\sum_{n=1}^{\text {Rows }}(\text { length_on_row })
\end{aligned}
$$

\subsubsection{Reel Motors}

The system includes a reel (Figure 6.10) that needs to be motorised so that the robot can not run over its own cable. The weight of the robot is $20 \mathrm{~kg}$, the weight of the reel without cabling is $15 \mathrm{~kg}$ and the approximate weight of cable is $50 \mathrm{~kg}$. The cable weight is limited to permit a single person to carry the reel a short distance as well as taking into account the physical limitations apparent in the cable design (the $50 \mathrm{~kg}$ limit allows for cables rated between $200 \mathrm{~kg} / \mathrm{km}$ and $165 \mathrm{~kg} / \mathrm{km}$ for a $250 \mathrm{~m}$ and a $300 \mathrm{~m}$ cable respectively). Thus the overall system has a maximum moving weight of $85 \mathrm{~kg}$, or $834 \mathrm{~N}$ when

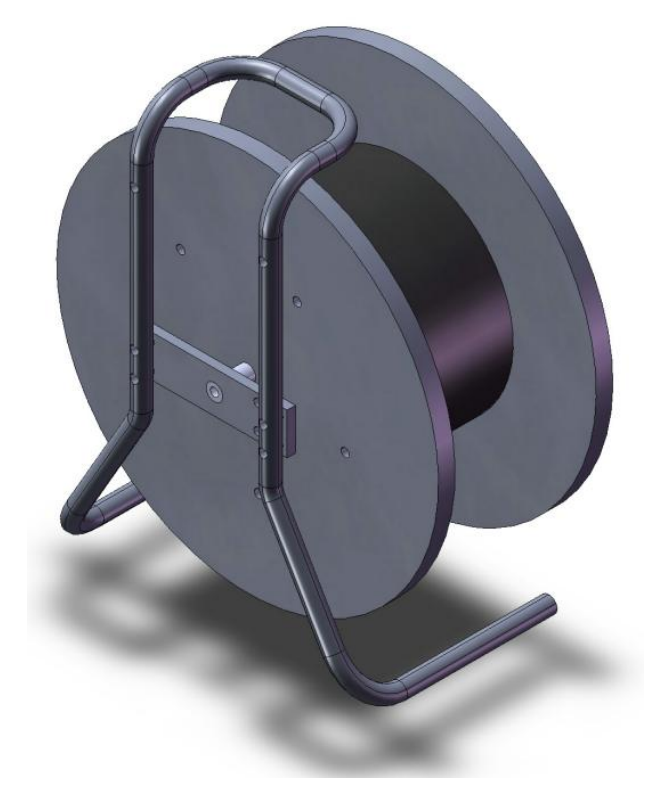

Figure 6.10: Current reel which has been stripped and cleaned to remove rust and check integrity 
converted to a force to allow for a torque to be determined. This force of $834 \mathrm{~N}$ is increased to $1000 \mathrm{~N}$ for specifying of the motor requirements to allow for friction and drag of the cable. It should be noted that the motorised reel is not designed to pull the robot out of the pipe and hence should never have to pull the $20 \mathrm{~kg}$ robot, but if the robot does fail then it would be useful for the reel to help the technicians pull the robot out. The torque required from the motor is calculated at the worst case scenario of the system, this is when all the weight is mounted at the extreme edge of the reel, hence the minimum torque required by the motor is $230 \mathrm{Nm}$ as the radius of the reel is $0.23 \mathrm{~m}$.

The RPM required from the motor is determined by the maximum speed that the robot is able to travel in the reverse direction. This is determined by attaching the largest set of wheels to the robot, raising the unit from the ground and recording the speed of rotation. The motor was directly connected to a $24 \mathrm{~V}$ supply and run continuously with 1 minute measurements taken regularly. After 10 such measurements, the maximum speed of the motor was determined to be $44 \mathrm{RPM}$. Hence with the large $85 \mathrm{~mm}$ diameter wheels the maximum speed of the robot is $11.75 \mathrm{~m} / \mathrm{min}$. Using a speed requirement of 15 $\mathrm{m} / \mathrm{min}$ as a design safety margin, the maximum continuous speed of the motor can be specified as 10.4 RPM by dividing the speed by the circumference of the reel (diameter of $0.46 \times \pi$ ); and the no load speed can similarly be specified as 40 RPM using the diameter of the reel drum (black section in Figure 6.10).

Using these specifications, a ratio formula has been inserted into a spreadsheet such that any motor can be tested easily to see if it is able to supply the necessary torque and speed requirements. This is done by determining the ratio using the required speed of the reel and the actual speed of the motor (equation 6.8) then taking this ratio to determine the maximum torque that the system 
would have with that motor (equation 6.9).

$$
\begin{aligned}
\text { Ratio } & =\text { Actual_Speed } / \text { Desired_Speed } \\
\text { Final_Torque } & =\text { Actual_Torque } / \text { Ratio }
\end{aligned}
$$

A series of 15 high end $12 \mathrm{~V}$ motors sourced from Faulhaber[37] as well as the standard suppliers of the university $[35,34,25,36,30]$ have been checked with this spreadsheet and found that none would be suitable. $12 \mathrm{~V}$ motors were checked as the transformer in the base station provides a $12-15 \mathrm{~V}$ output as well as a $300 \mathrm{~V}$ output used to power the robot. The $300 \mathrm{~V}$ lines have been avoided as motors are noisy devices and bringing additional noise into the robotic section of this system is undesirable. At the present time, high voltage DC motors have not been tested nor have single phase AC motors. Single phase AC motors are an option as the transformer is powered via a single phase generator. This would be the best option as one stage in the base station transformation is an isolating transformer, hence by using an AC motor the noise from this motor can be isolated from the base station and the robot without additional components being added.

\subsection{Hardware Summary}

The lights used for this robotic unit are high power white LEDs housed in custom made, watertight holders. These LEDs provide full illumination of the pipe while keeping colours consistent.

The two camera options in this project both provide resolutions of greater that 460 TVL in PAL format and have a maximum zoom of $120 \times$. The Sony FCBH11 high definition camera surpasses all researched pipe inspection systems in resolution and equals the best in zoom ability. Additionally both cameras 
are able to be run via a serial connection by taking advantage of the VISCA ${ }^{\mathrm{TM}}$ protocols.

The Sabertooth $2 \times 5$ and SyRen10 motor drives provide all the required control for the camera and drive motors inside the robot. These drivers are able to supply at least twice the current drawn by the motors attached. Additionally voltage spikes will not damage the motor drivers due to the voltage over specification.

The Logitech Extreme 3D Pro joystick has three 16 bit axes which allows for full control of the robot with high precision.

Replacements for all motors and gearboxes inside the robot have been located. The fitted motor works smoothly with the older devices, thus reliability of the robot is maintained.

Replacement cabling options have been found. A spreadsheet designed to calculate the amount of cable able to fit on the reel has been created, thus determination of which cable is the most suitable option is now possible.

The speed and torque requirement of a reel motor have been determined and a spreadsheet exists for determining if a motor is suitable. The spreadsheet also gives the required gearing ratio to make the motor work in the system. 


\section{Chapter 7}

\section{Software}

A Graphical User Interface (GUI) is required to allow the user to easily interact with the robotic unit during inspections.

Microcontroller code is also required so that the microcontrollers are able to perform the desired functions including controlling the internal components of the robot, reading the reel encoder and controlling the motorised reel. As previously mentioned, the rotary encoder is used to determine the distance travelled by the robot, and the motorised reel prevents the robot running over its own cable.

The pipe inspection system is designed in a hierarchical fashion with the user input at the top of the chain and the robotic vehicle at the end (shown in Figure 7.1). In Figure 7.1 the main components are distinguished by rectangular boxes, and it is these components that code must be written directly for. The sub-components are those in the diamond boxes, which are either controlled or read by the main components. The microcontroller code will be written in $\mathrm{C}$ using the Integrated Development Environment (IDE) HiTide due to its availability and compatibility with the microcontroller used. 


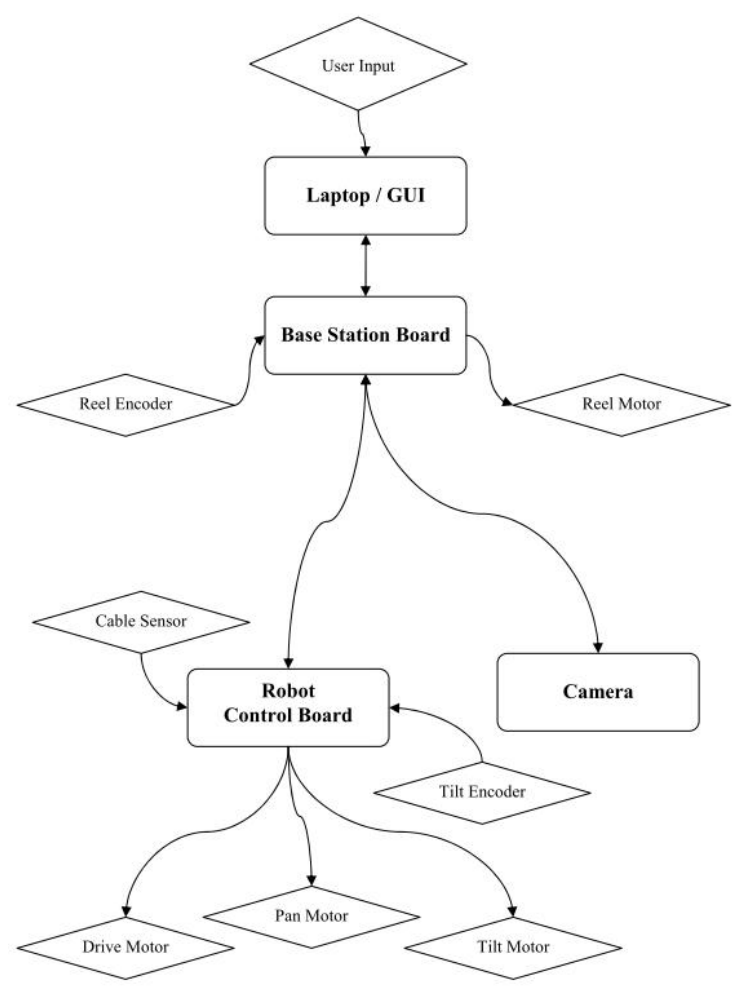

Figure 7.1: System hierarchy showing main components in rectangular boxes and sub components in diamond boxes.

\subsection{C\# Graphical User Interface}

Ristic endeavoured to convert the GUI from the original LabVIEW code (section 2.3) into a new form using Microsoft Visual Basic (VB) (VB.NET framework). He did not complete this conversion and when attempts were made to modify his code, multiple run-time errors were encountered.

The decision was made to write the GUI from scratch using C\#. C\# has the advantages of allowing for easy implementation of plug-ins for Microsoft Windows [45]. The IDE Microsoft Visual Studio (MS VS hereafter) is used to create and compile the code for the GUI. This IDE compiles the project into an executable file, meaning for control of the robot the user only requires the folder containing the executable file and the additional components used in the design. The components required to run this GUI are the DirectShow and DirectX plug-ins which are freely available for Microsoft Windows (http: 
//www.microsoft.com/downloads/en/default.aspx). With these it is possible to capture video and to interface a game controller which are explained further in the sections that follow.

\subsubsection{Revision 1: C\# Implementation}

\section{Conversion}

The first revision of the robot control program involved taking the incomplete VB form started by Ristic and implementing all the functions for the buttons in C\#. A form is the name given in VB and C\# for a code file, these forms contain both a front panel or design and a code section. When a button or region is added to the front panel (Figure 7.2) of a form in MS VS, skeleton code is automatically created in the code writing section (Figure 7.3) of the project.

MS VS has the capability of converting a VB project to a $\mathrm{C \#}$ project and vice

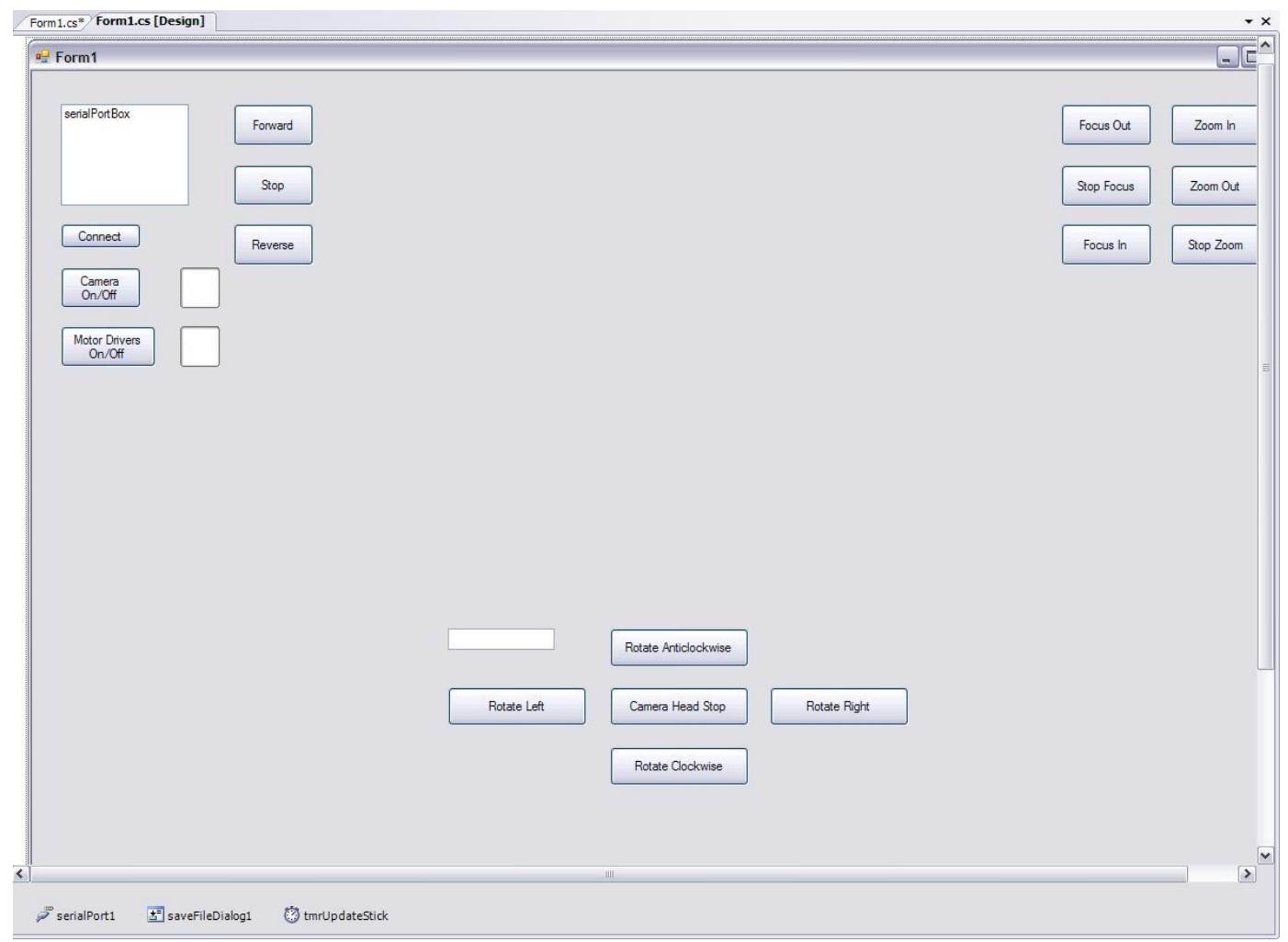

Figure 7.2: C\# front panel of form. 


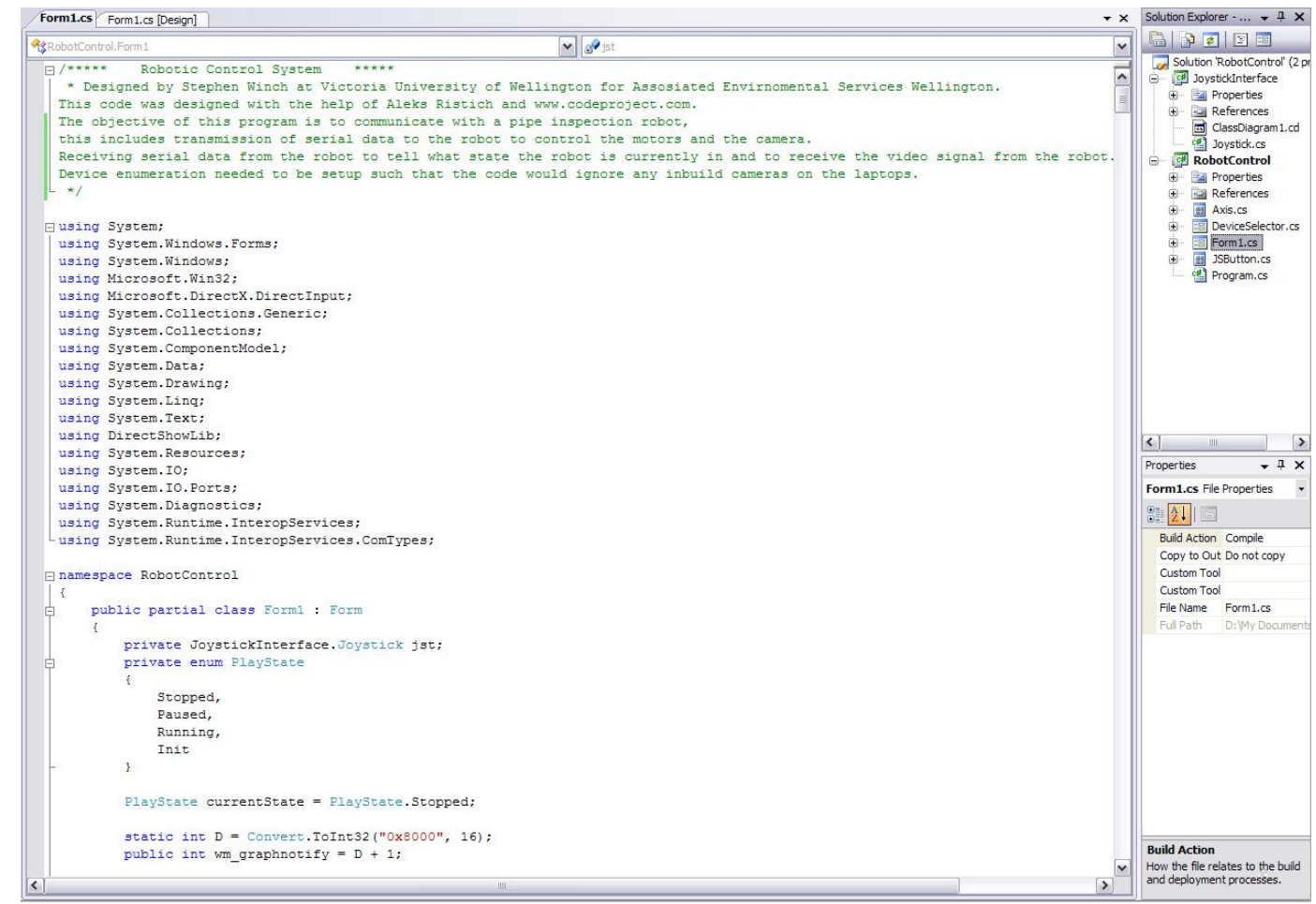

Figure 7.3: C\# Code section of form.

versa, although some syntax errors are introduced. Once these errors were corrected, the form contained all the buttons, indicators and display regions included by Ristic (Figure 7.4). Of these, Ristic had linked "power on" buttons to their respective indicators, implemented the serial communication and motor movement functions. Hence the first objective in relation to the control program was to implement the buttons for camera control, adjust functions where necessary and implement the video display.

The focus buttons located at the top right of the GUI are connected to functions that send the required serial packet to the camera via the robot. The values sent are $8101040702 \mathrm{FF}, 8101040703 \mathrm{FF}$, and $8101040700 \mathrm{FF}$ for focusing inward, outward and stop focusing respectively. Figure 7.5 is the code used for focusing of far away objects. Similarly the zoom buttons (also located at the top right) are connected to functions that send the camera the control data packets of $8101040802 F F, 8101040803 F F$, and 8101040800 FF for the zoom inward, outward and stop zoom functions of the camera respectively. The data packets 


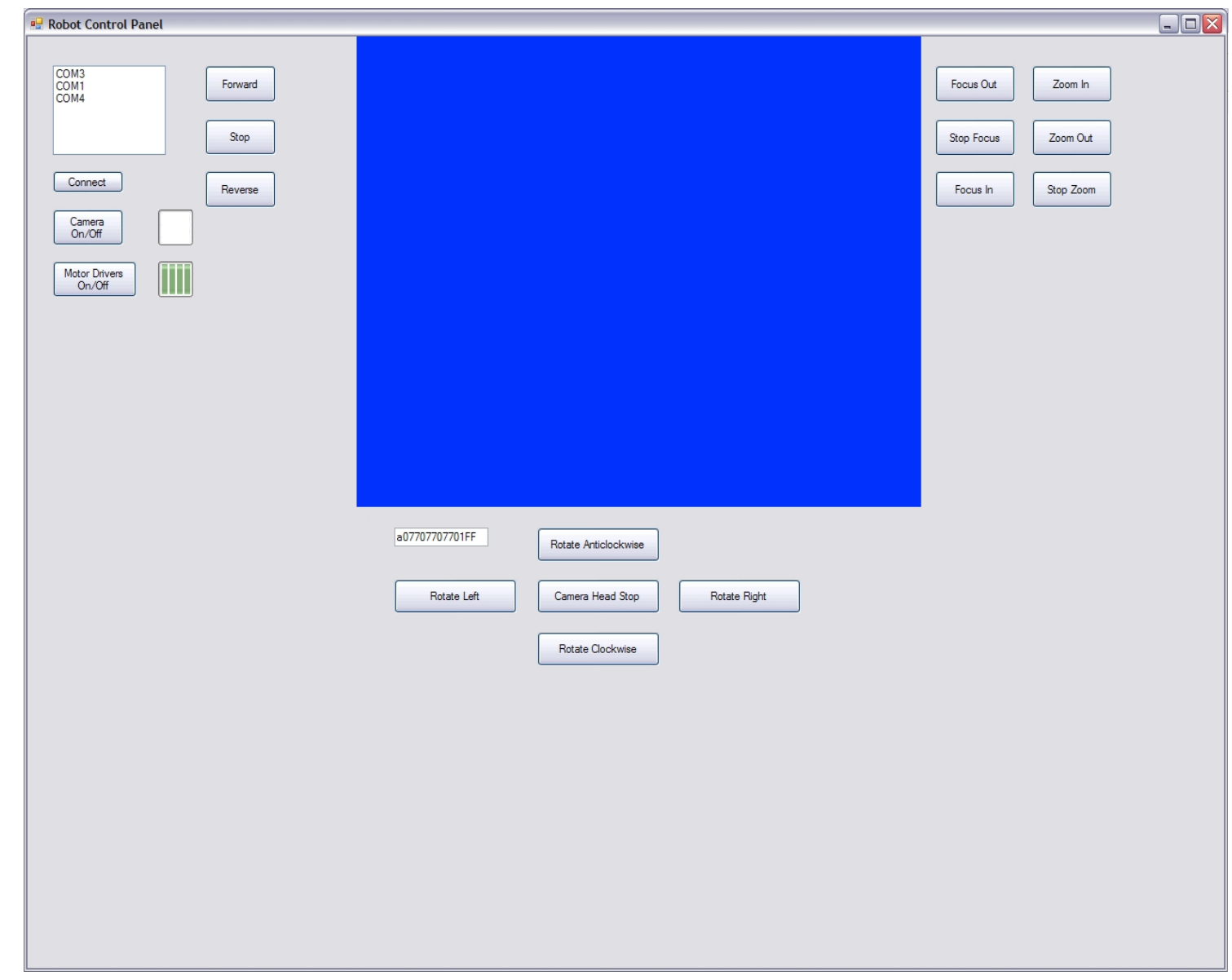

Figure 7.4: GUI Revision 1: Implementation

required to perform each of these functions was acquired from Ristic [11, p.61] and cross checked with the Sony EVI-371 datasheet which can be found on the Appendix CD.

The data box inserted at the top left of the program displays the serial data ports of the PC. It is implemented to automatically connect to the newest (last) connected port as the first ports located are likely to be connected to components such as external USB keyboards and mice. The connect button positioned below this dialogue box will refresh the port list and reconnect to the last serial device connected. This is implemented so that it is possible to connect the robot after the program is running. If the program does not recognise another device (such as a connected camera or phone) then an error message will appear at start-up indicating that it was unable to locate the robot and asking the user to connect the robot and restart the program. After the user clicks 


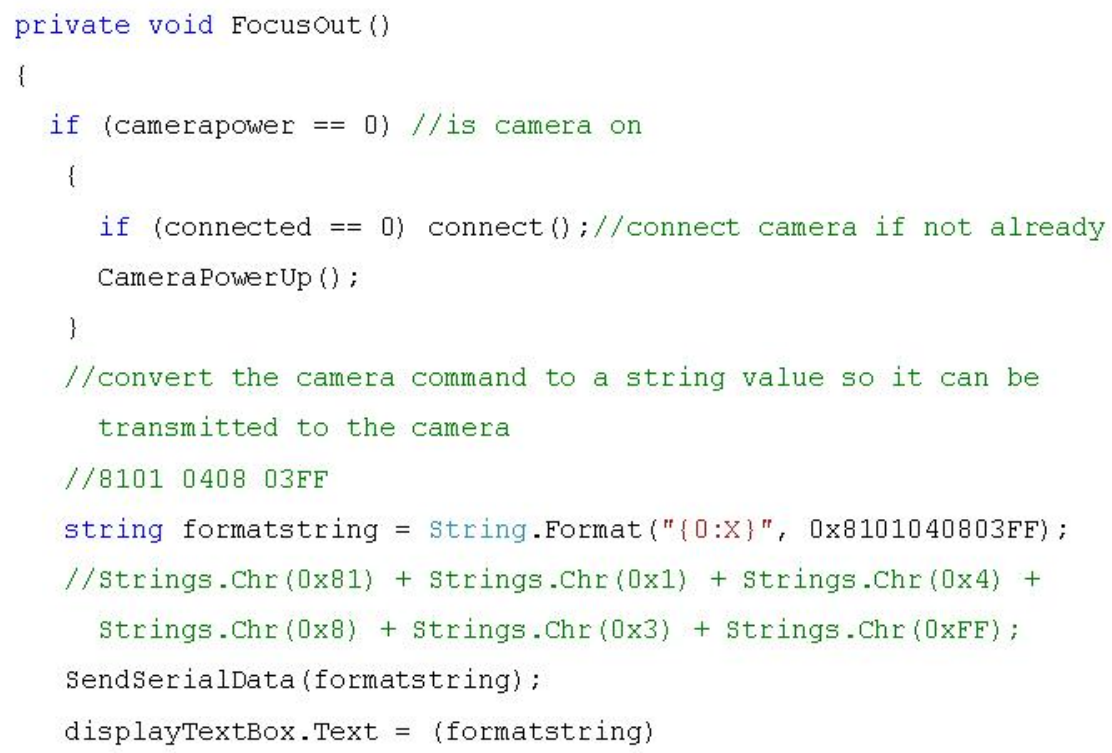

Figure 7.5: Code for transmission of "focus far" command to camera.

the OK button on this message, the program closes releasing all resources that it has allocated.

\section{Robot Control Functions}

The three buttons located beside the dialogue box are used for driving the robot forwards, backwards, and stopping the robot. The topmost button is used to drive the robot in the forwards direction, where each time the button is clicked the speed at which the robot moves is increased linearly. The value sent to the robot ranges from 077 to 130 where 077 is the point at which the robot is stopped and 130 is the point at which the robot is moving forward at full speed. The middle button (labelled Stop) restores the control value to 077 which will stop the robot from moving forwards or backwards. The bottommost button in this group is used to reverse the robot by sending values between 013 and 077 where 013 causes the robot to reverse at full speed. Again each click of this button causes the speed to increase linearly in the reverse direction. Limitation conditions have been included at both the start and end of the range to prevent the motor from incrementing to a value that would 
be unrecognisable by the robot. If the values sent to the robot are at the extremities of the storage registers, communication or interpretation errors can cause large changes in motor control values, hence absolute maximums and minimums are not used.

The camera motor control buttons located underneath the video window, are the " $+"$ style setup in Figure 7.4 to give the appearance of 2 degrees of movement. In the centre is the camera stop button which is designed to stop all movement of the camera by sending the robot the values of 077 for both the camera motors. The four functions connected to the buttons around the stop button are setup in the same way as the drive motor control in that they have a high value of 130 and a low value of 013 . The rotate left and rotate clockwise buttons increment down the values, that is, they operate between the values of 077 and 013 whereas the rotate right and rotate anti-clockwise buttons increment up the values, operating from 077 to 130 . The value is then written to a character string relating to the camera tilt and pan motors respectively.

At the end of every control function related to the movement of the robot (not to the control of the camera) an updated data packet is transmitted to the robot and also displayed to the user in the text box underneath the video window. The data packet is explained in detail in section 7.1.1.

\section{Video Capture}

As the robot visually inspects the pipes, video acquisition and display is an integral part of the design. The video capture is handled in a six step process [46]. The first step prompts the user where they would like the file saved to and what they would like to call the file. The file can only be saved as ".avi" as compression filters in the code have not yet been setup.

The second step is to acquire the appropriate interfaces from the DirectShow library and the operating system so that the program will be able to access the 
devices and procedures that it requires to capture the video stream.

The third step is to find all possible capture devices and ask the user to select the appropriate one if more than one exists. As a large number of modern laptops come with an inbuilt web camera it is necessary to have a method to bypass using such cameras. This required the implementation of a device selection method which iterates through all connected video capture devices and creates a selection list so the user can select the desired device.

Step four is setting up the device so that it is able to record and display the captured video signal. To do this, types of DirectShow methods need to be used to capture and build the video "graph" and its corresponding filters. This graph needs to be rendered either to the preview window or to the file. This preview is of significantly lower quality than the video saved as the computer is otherwise unable to process it in real time.

The fifth step is setting up the preview window so it is able to fit the GUI designed. This requires using C\# standard form methods to set the position and size of the window.

The final step in the video capture is closing the video (Figure 7.6) and releasing the devices and resources (Figure 7.7) so they are able to be used by other parts of the system. If the closing is not completed properly then the video will not be saved as a complete file, and is likely to crash some media players. If the devices and resources are not released then the control program itself will crash on exit which usually has no side effects on the program other than not being user friendly. At worst case, not releasing some of the resources can cause Windows to lock up making it impossible to use without a manual reboot of the system. Hence proper closure of the program is a task that has been thoroughly explored and implemented. 


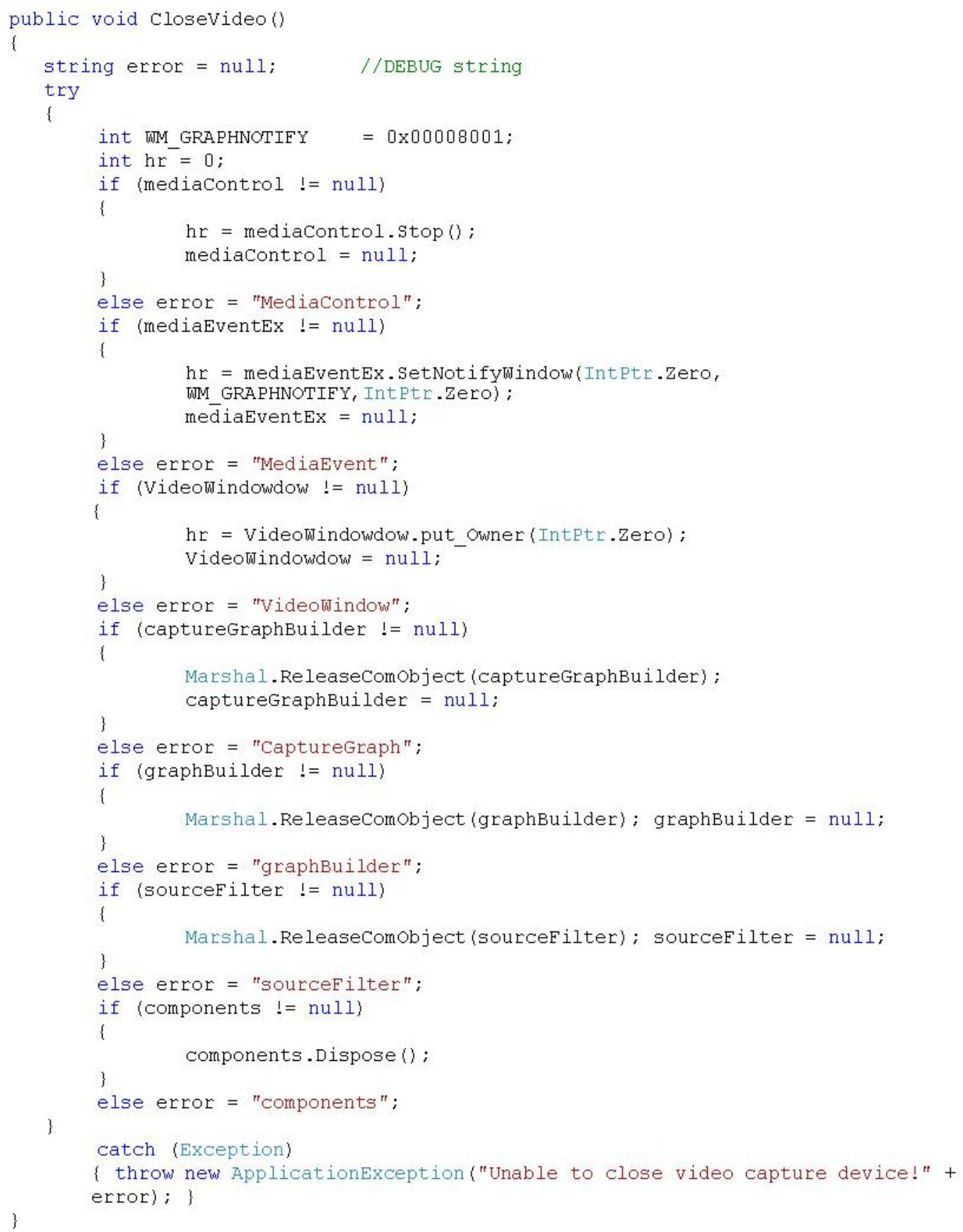

Figure 7.6: Code used to close the video stream on program exit.

\section{Serial Communication}

As there are multiple instructions that need to be sent to different peripheral components, packet data transmission is the most applicable method of communication as it allows for the use of automatic address recognition and large data packet sizes. Automatic address recognition is where each peripheral de- 


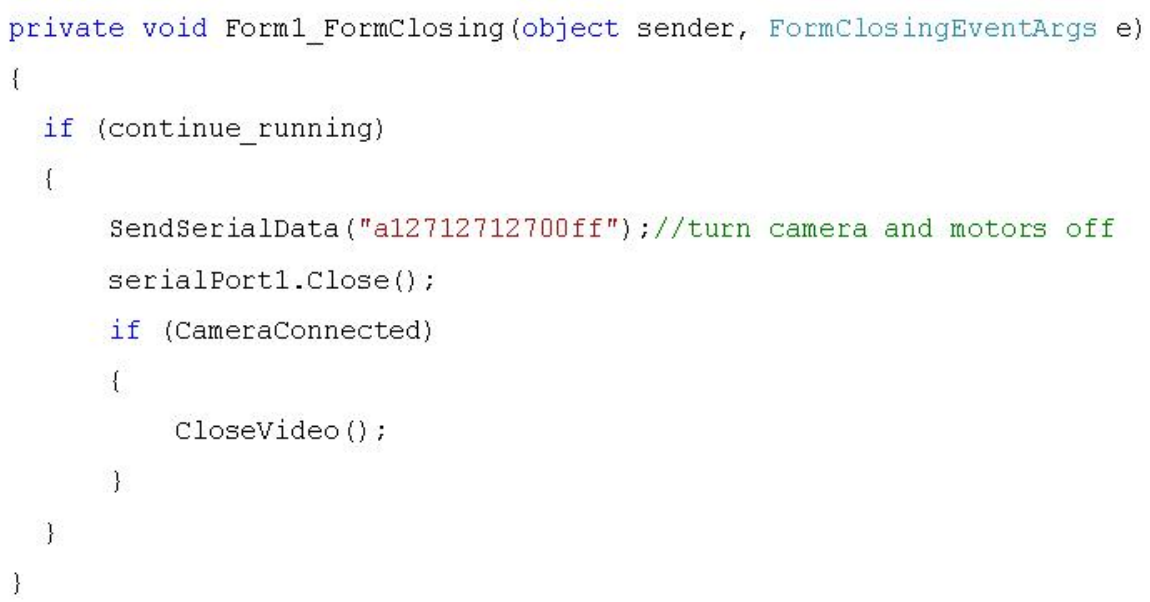

Figure 7.7: Method called when program closes, it closes the serial port then calls video closure method.

vice is assigned a start character to look for. If that character matches the start character of the incoming data packet the device knows that the data is designated for it, otherwise the device ignores the data. As the camera is also able to use serial data packet communication it is possible to connect both the robot and the camera to the same communication line with the data packet method. The camera has an inbuilt character recognition sequence of '8101', hence the robot cannot be assigned a character of 8 , and is instead assigned the character 'a'. The format of the data packets is ASCII due to their cross compatibility with the VISCA ${ }^{T M}$ data transmission protocol designed and used by Sony [47]. The possible control signals and these functions are shown in Table 7.1 in the correct format for transmission. These functions are sent at the instant of occurrence in the code unlike those for control of the robot (explained in section 7.1.1) as communication to multiple devices with one data packet is not possible and the camera can only perform one function per packet.

The data packet is created by adding the automatic address recognition character to the front, end characters to the end and the information required inbetween. This is done by creating a data string and transmitting that down the communication line. This data string has two different formats, the simplest is when the camera is being controlled (shown in Table 7.1), and the other imple- 
Table 7.1: Control strings and their corresponding function for Sony camera modules EVI-371 and FCB-H11

\begin{tabular}{ll}
\hline \hline Data String & Function \\
\hline $8101040702 \mathrm{FF}$ & $\begin{array}{l}\text { Focus out: used to manually adjust the camera focus } \\
\text { causing the camera to focus on a distant object. }\end{array}$ \\
8101040703FF & $\begin{array}{l}\text { Focus in: used to manually adjust the camera focus } \\
\text { causing the camera to focus on a nearby object. }\end{array}$ \\
8101040700FF & $\begin{array}{l}\text { Stop Focus: stops the adjustment of the focus of the } \\
\text { camera. }\end{array}$ \\
8101040802FF & $\begin{array}{l}\text { Zoom in: causes the camera to zoom in on a central } \\
\text { object. }\end{array}$ \\
8101040800FF & $\begin{array}{l}\text { Zoom out: causes the camera to zoom out from an ob- } \\
\text { ject back towards 1x normal outlook. }\end{array}$ \\
\hline
\end{tabular}

mentation is for controlling the robot. The latter implementation is more complex as more than one device is controlled by the main control board housed inside the robot. Each control value for each of the components inside the robot needs to be set, remembered by the program and transmitted to the robot. These controllable devices are the drive motor, the camera tilt and pan motors, and the motor's and camera's on/off switches.

The motors are controlled by Pulse Width Modulation (PWM) signals via a motor driver (see section 7.2.1 for coding details or section 4.2 for hardware configuration). To create these PWM signals, values representing duty cycle levels are sent to the robot's microcontroller which then converts them to a PWM. This requires a set of 3 characters for each of the 3 motors. The control values for the switches need to be set as a high or low, hence 2 one character values are assigned. The resultant is an 11 character data string to be transmitted to the robot with the addition of end characters and an automatic address recognition character. This results in equation 7.1, where "a" is the automatic address recognition character and "FF" the end characters.

$$
\begin{aligned}
\text { Data }= & =a^{\prime \prime}+\text { main_mtr }+ \text { head_mtr_1 }+ \text { head_mtr_2 } \\
& + \text { cam_power }+m t r \_p o w e r+" F F^{\prime \prime}
\end{aligned}
$$


Two end characters are used to prevent the receiving device from ending midstream if it comes across one of the end characters inside the data packet.

The serial communication had been implemented in LabVIEW by Ristic and hence converting them to $\mathrm{C \#}$ was the first step in implementing the robotic control GUI.

\subsubsection{Revision 2: Joystick Adaptation}

The second revision of the control program consisted of making the interface more user friendly. This involved connecting and calibrating a joystick in order to control all functions and movements without using the keyboard or mouse. To use the joystick, the DirectX plug-in is required as this provides the basis for the acquisition of the device as well as the methods required to understand the data being sent to the computer by the device. The joystick used is a Logitech $\mathbb{R}$ Extreme $^{\mathrm{TM}}$ 3D Pro which is a 3-axis 12 button joystick with a throttle control and Point Of View (POV) "hat". Of these functions, only the 3-axis movement and 10 of the 12 buttons are used. The 3 axis of movement allows for the robot to be driven by moving the yoke in a forward or backward motion, tilting of the camera head clockwise or anti-clockwise by pushing the yoke left or right respectively, as well as allowing for the camera head to be panned left or right by twisting the yoke in a anti-clockwise or clockwise motion. The driving brake, which prevents the robot from moving forward or backward, is attached to the joystick trigger and the camera head brake which prevents the camera head from turning is attached to button 2 located on the side of the yoke (Figure 7.9(a)). Buttons 3 and 4 (Figure 7.9(b)) on the yoke are used to control the zoom far and near functions of the camera respectively. These are the most extensively used commands in the day to day operation of the robot and hence this button selection is designed to allow for easy access to these functions. 


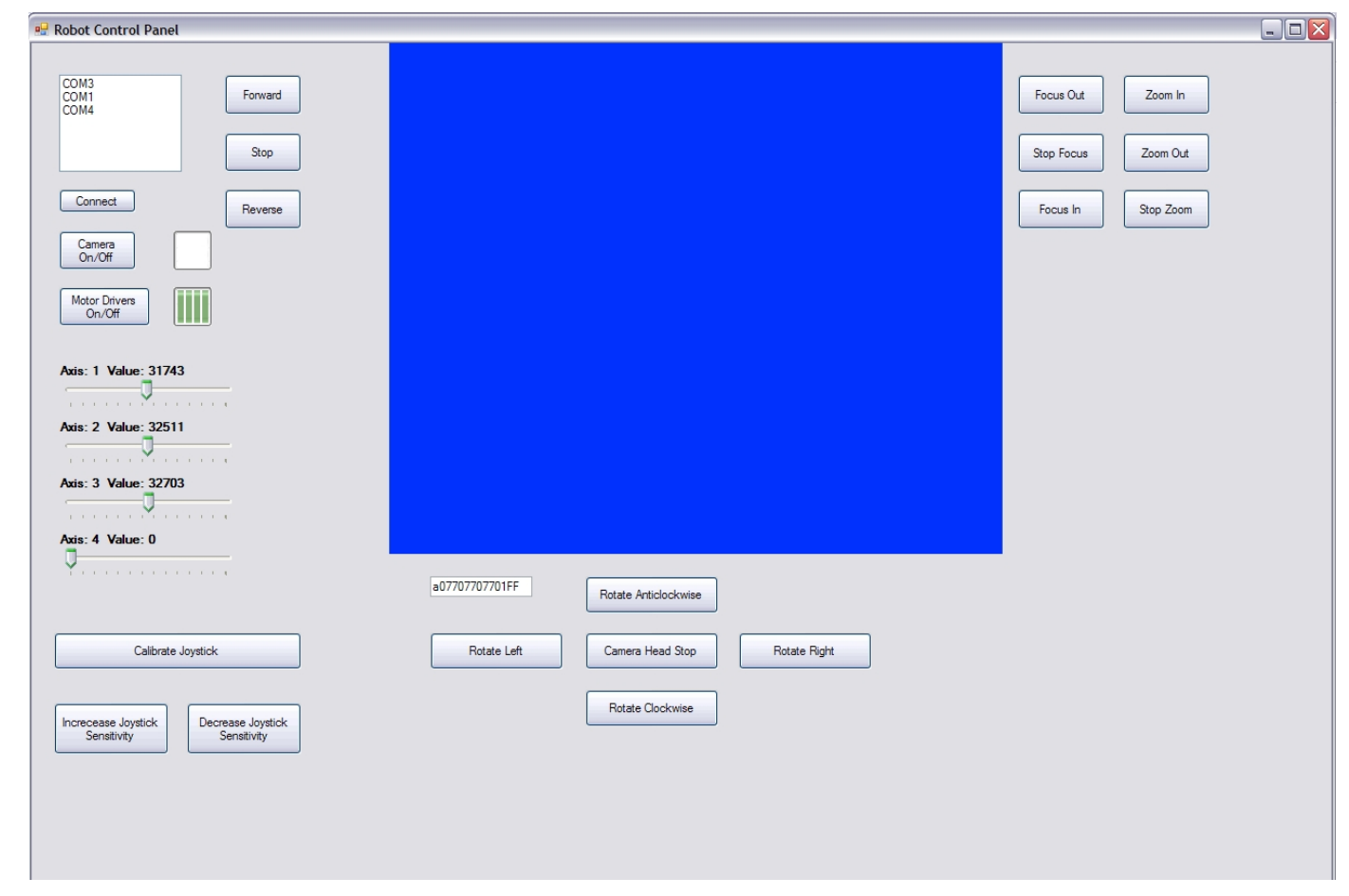

Figure 7.8: GUI Revision 2: Joystick Calibration

The buttons located on the base of the joystick (Figure 7.9(c)) are used for camera focus and joystick calibration. Buttons 7 and 8 have been programmed to control the far and near focusing option of the camera respectively. Although the camera is an auto focus camera, it also contains the option of manually adjusting the focus in case the camera is focusing on the wrong object. Buttons 9 to 11 inclusive are used to calibrate the joystick, allowing for correction of any off-set in the yoke position caused by movement during the original calibration stage without restarting the program. Buttons 9 and 10 are used to decrease or increase the sensitivity of the yoke respectively. Button 11 is programmed as a joystick recalibration button which causes the joystick to recalibrate its zero point values to the current location of the yoke. The final button programmed on the base of the joystick is button 12 , which functions as a panic button, causing both the camera head motors and the drive motor to brake and come to a complete stop.

For the calibration of the yoke, the raw axes values taken from the joystick were displayed in order to derive a formula for converting the joystick input 


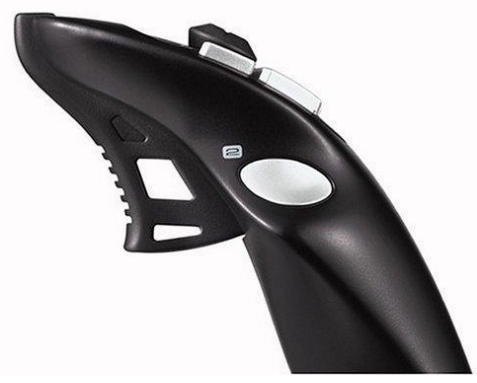

(a)

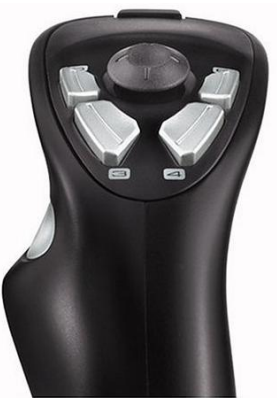

(b)

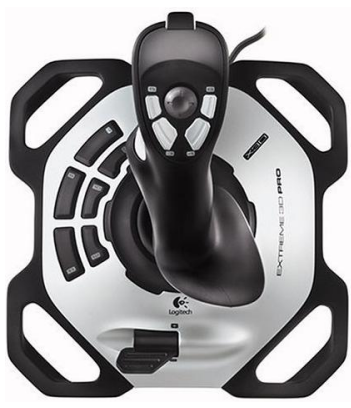

(c)

Figure 7.9: Locations of buttons on the Logitech Extreme 3D Pro joystick including buttons (a) 2, (b) 3-6 and (c) 7-12; the trigger is button 1[48].

to the values required for transmission to the robot and control of its motors. With the forward and backward movement (axis 1), sideways movement (axis 2) and rotational movement (axis 3) data displayed (Figure 7.8) it was possible to plot a joystick input versus required output.

Axes 2 and 3 move in the same direction as the desired movement of the robots head, thus a plot was produce to acquire Equation 7.2 for a positive linear relationship between the joystick input and the desired output. Axis 1 is the exception. As joysticks are configured for flight control in games, a backwards pull on the yoke increases the value sent to the computer. This is opposite to the desired control of the robot, hence a plot was produced to acquire Equation 7.3 for a negative linear relationship.

$$
\begin{aligned}
& \text { Output_Val }=(\text { Input_Val/526) }+13 \\
& \text { Output_Val }=136-(\text { Input_Val } / 526)
\end{aligned}
$$

The intercept values of 136 and 13 correspond to the maximum and minimum speeds that are to be sent to the motors. The absolute minimum, zero, is not used in order to prevent errors in coding or understanding by the microcontroller causing the robot to act unexpectedly. When a register on the microcontroller is passed a negative value it will loop around to its maximum. This causes a negative value to be interpreted as a high positive value. The reverse 
case is also true, where an input value of 256 will cause these 8-bit registers to reset to zero. Thus by avoiding the extremities of the registers this error cannot occur.

The control signals sent to the robot need to be modified to allow for greater speeds from the robot without significantly reducing the precision of control of the robot. This requires the modification of equations 7.2 and 7.3 to utilise the full spectrum of the 8 bit PWM, thus the maximum, minimum and mid values of the control signals should be set to 255,0 and 127 respectively. Greater speed control is possible using equations 7.4 and 7.5 in the GUI.

$$
\begin{aligned}
& \text { Output_Val }=\text { Input_Val/256 } \\
& \text { Output_Val }=255-\text { Input_Val } / 256
\end{aligned}
$$

The joystick was tested with the robot and small errors were found when the joystick was fully pushed in one direction. This was determined as a similar overflow error as stated previously in revision 1 (section 7.1.1) and therefore new buffer regions were incorporated into the system. These buffer regions test if the robot is being sent a value less that 5 . If so the value is set to 5, giving a lower buffer region. The reverse case is handled by using a similar if statement to test when the robot is being sent a value greater than 250 , if so the value is set at 250 to give the upper buffer region.

Each digit in the control signal is sent as an ASCII character (as described in section 7.1.1) thus decoding of the signal by the microcontrollers on the base station and the robot itself must be implemented (sections 7.2.2 and 7.2.1 respectively). 


\subsubsection{Revision 3: Indicators}

In this revision of the program, speed and location indicators have been added to the GUI (Figure 7.10). These indicators allow for the user to easily see how fast each motor is moving and where the camera head is currently positioned with respect to its starting position. In this coding, two horizontal progress bars have been added to display the speeds of the camera motors, two 'track' or slide bars have been added to display the camera head location and one vertical progress bar was added to indicate the speed of the robot itself. The horizontal progress bars and the track bars are standard functions in the C\# program. The vertical progress bar is a custom tool and needs to be created as a separate class before it can be added [49]. The properties of the progress and track bars have been modified to reflect their range of operation and function. All are named after their respective motors and range from 0 to 255 .

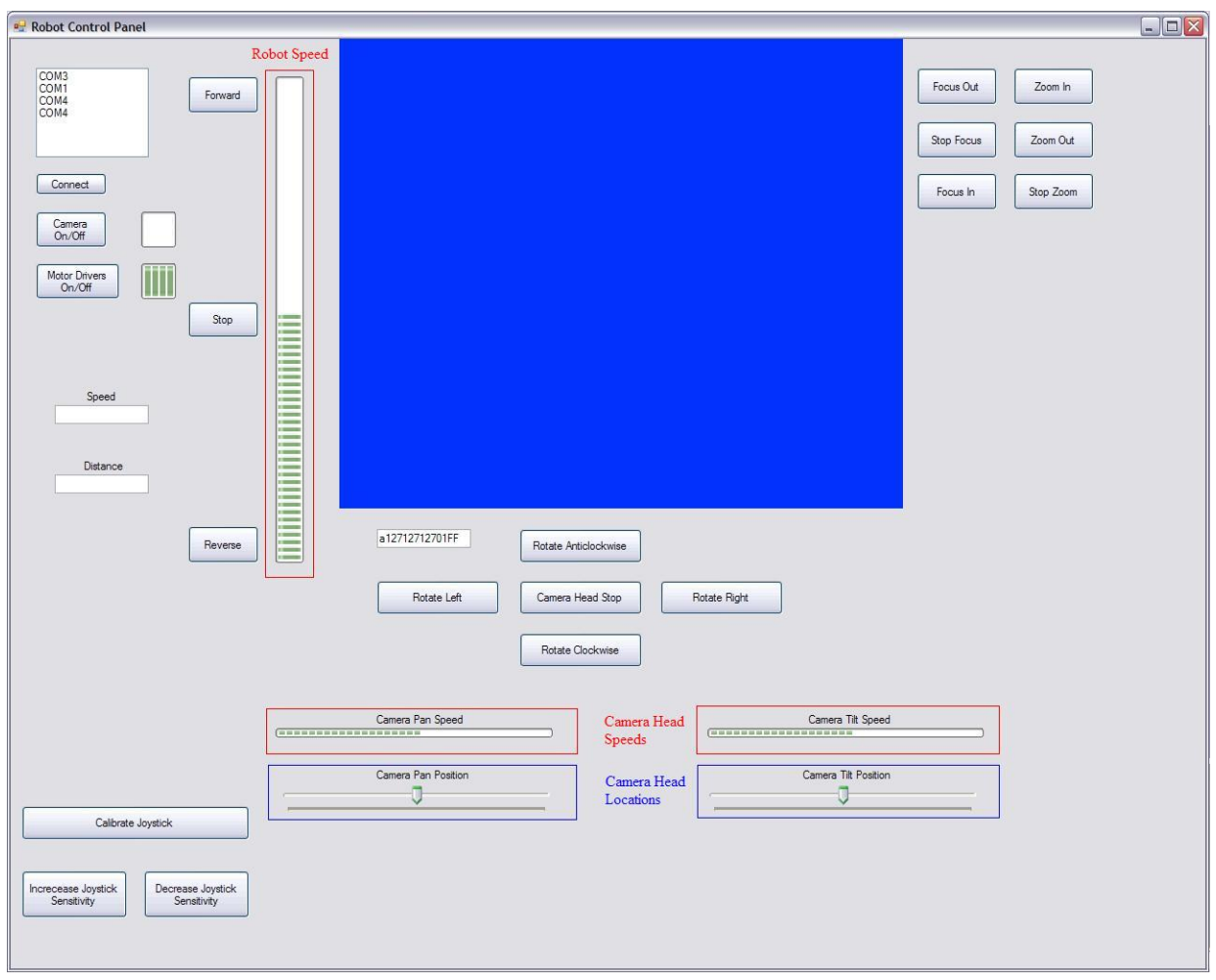

Figure 7.10: GUI Revision 3: Progress bars and position indicators incorporated. 
Feed back from the robot is currently unimplemented, hence the track bars have not been coded.

\subsubsection{Revision 4: Video Recording \& Relocation}

Recording of the video to the hard drive of the computer is required so that the inspection can be analysed at a later date. The problem with recording is that video compression is required as uncompressed video will take up 70 GB of hard drive space for a one hour long recording with the older Sony EVI371 camera (see datasheet on the Appendix CD for camera details, Figure 7.11 for size versus time plot). Recording with the more advanced Sony FCB-H11 camera (datasheet on the Appendix CD) increases the size of the video due to its 2 mega-pixel resolution. When this camera is used in HD mode each of the three component lines has an 8-bit (one byte) depth for each of the 2,000,000 pixels. Hence a video with $6 \mathrm{MB}$ frames at the lowest frame rate of $24 \mathrm{fps}$ will create a video of $144 \mathrm{MB} / \mathrm{s}$ in size. This requires considerable compression as

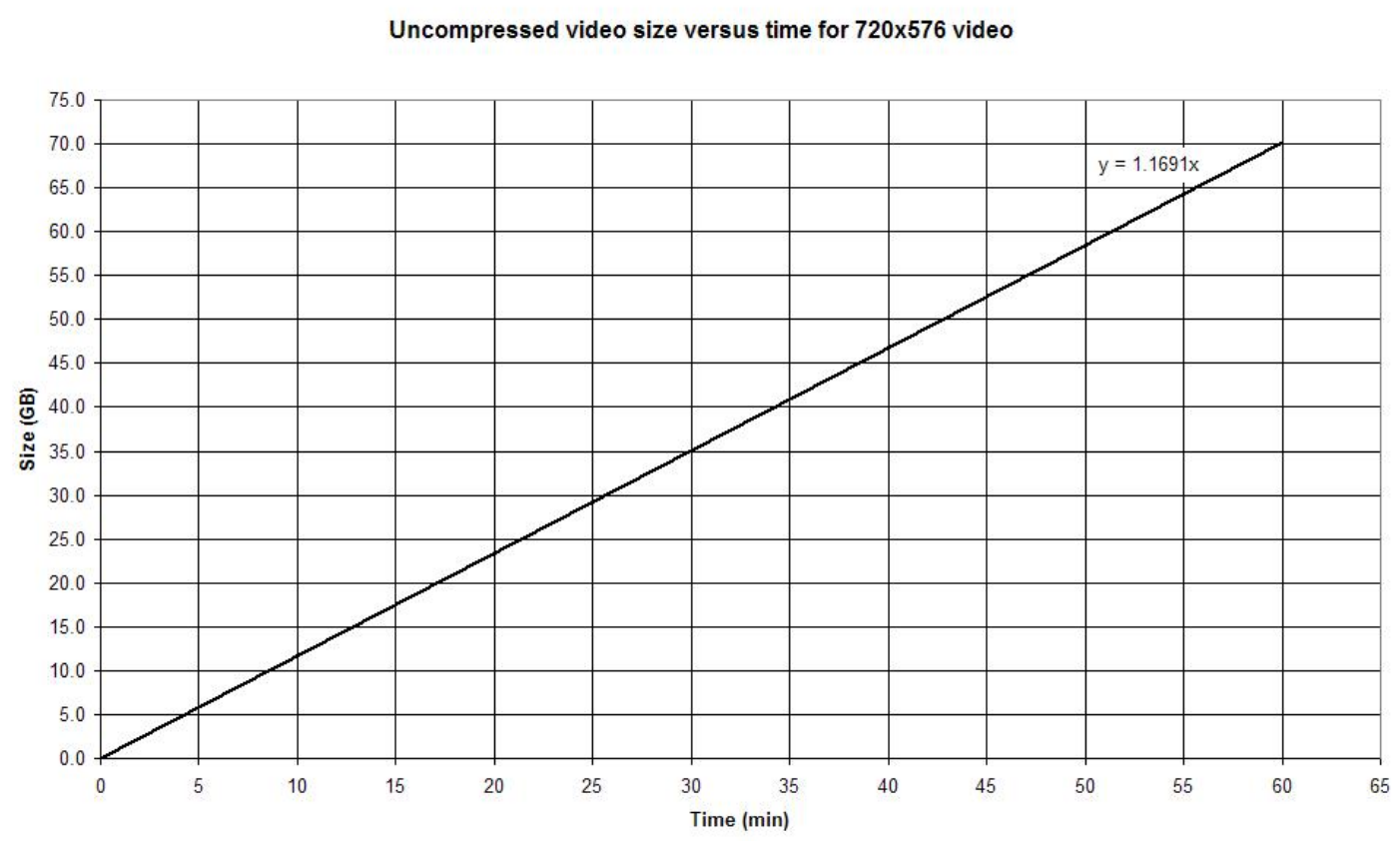

Figure 7.11: Uncompressed video size versus length of recording for Sony EVI371 camera module 
a 1 hour long inspection would encompass a 518.4 GB portion of a hard drive.

Two possibilities for the implementation of video compression exist. The first is to use a software compression algorithm which will require the redesigning of the code for the GUI as the system will need to be split into separate classes such that multi-thread programming can be implemented. This is due to the current setup causing the computer to run out of CPU power hence lagging (video recording setup is explained further on in this section). As modern computers consist of multiple cores each being able to run one or more threads (the new Intel i3, i5 and i7 series processors are able to run two threads on a single CPU), creating a program that is able to run multiple threads would be an efficient way to compress the incoming video stream in real time. A high definition video capture device would be needed to use the HD output from the Sony FCB-H11 camera acquired. The most suitable device found is a USB Hauppauge HD PVR which retails for USD\$199 and requires a 2 GHz CPU with $256 \mathrm{MB}$ of dedicated graphics.

The second option is to use an external hard drive recorder to record the incoming video stream. This setup allows for easy recording of the video stream with high end video compression techniques as the unit would be a commercially designed unit sourced from one of the leading video hardware companies. The hardware video compression technique would allow for "on the fly" video compression as the process of compression would be carried out by dedicated video processors designed specifically for this purpose. Acquiring a system that allows for hard drive expansion and removal would allow AES to easily transfer data from the field teams to the analysts back at the office as the hard drives would simply be able to be switched out. The video would still be displayed on the computer screen for control of the robot by using either a video cable splitter or taking the monitor output from the hard drive recorder and feeding this through an adaptor. The use of an external hardware video 


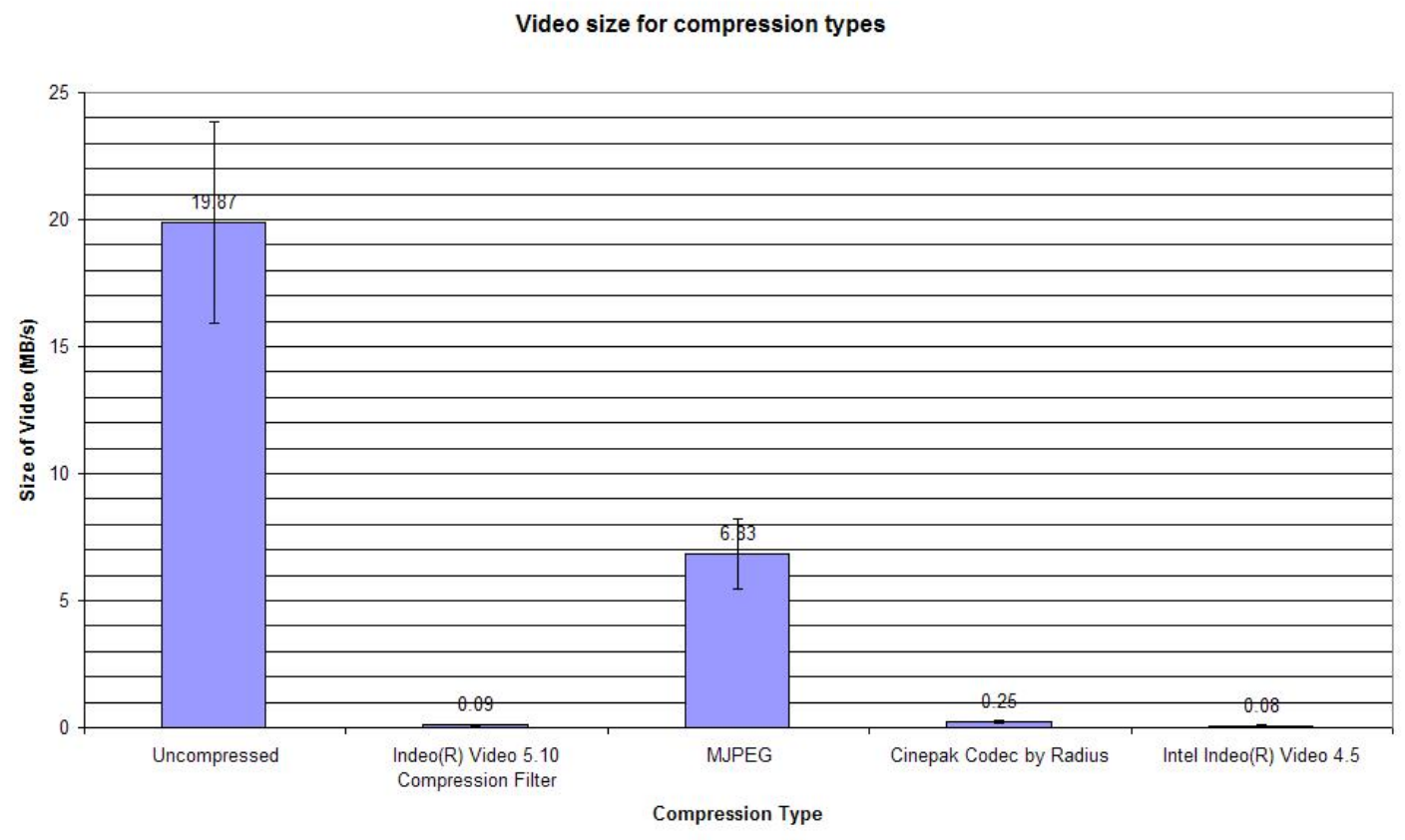

Figure 7.12: Video Compression Comparison

compressor and recorder allows for the possibility of using HD video, which is one of the possible outputs of the Sony FCB-H11 camera. Hardware recorders have an initial cost of NZD $\$ 500$ or more and the units are usually bulky. Due to the easy of implementation versus the portability of the system, both software and hardware options are being considered.

Four high image quality compression codecs are able to be implemented in MS VS. These are Indeo Video 5.10, Intel Indeo Video 4.5, Cinepak Codec by Radius and MJPEG. To find which codec would provide the best compression, a comparison was made by recording a 30 second test video (Figure 7.12). From this the two Indeo codecs were found to provide the most compression with MJPEG providing the least. MJPEG is still considered as it provides a video $\frac{1}{3}$ the size of the raw video and is not severely affected by lagging unlike the others. Lagging occurs in the compression as the program exhausts its allocated CPU allowance, MJPEG only lags during preview as recording takes precedence. Therefore, the most applicable software compression method at the current time is to use MJPEG and possibly reduce the frame rate of the 
Table 7.2: Size of video produced per run and daily

\begin{tabular}{lcc}
\hline \hline Compression Type & Size per run (GB) & Size daily (GB) (6 runs) \\
\hline Uncompressed & 518.4 & 3,110 \\
Indeo(R) Video 5.10 & 2.4 & 14 \\
MJPEG & 180 & 1,080 \\
Cinepak Codec by Radius & 6.5 & 39
\end{tabular}

1 run $=1$ hour of recording

preview.

Using MJPEG compression, a one hour long recording would require approximately 180 GB, whereas a Cinepak compression would result in a 6.5 GB video file and a Indeo compression would result in a 2.4 GB video file. Thus finding a way in which it is possible to use one of the Indeo video compression codecs would be a preferred solution for this project as a standard day for the field technicians consists of 4 - 6 inspections, each taking a maximum of 1 hour. As the two Indeo video codecs are almost identical one has been used when comparing the types of video compression (Table 7.2).

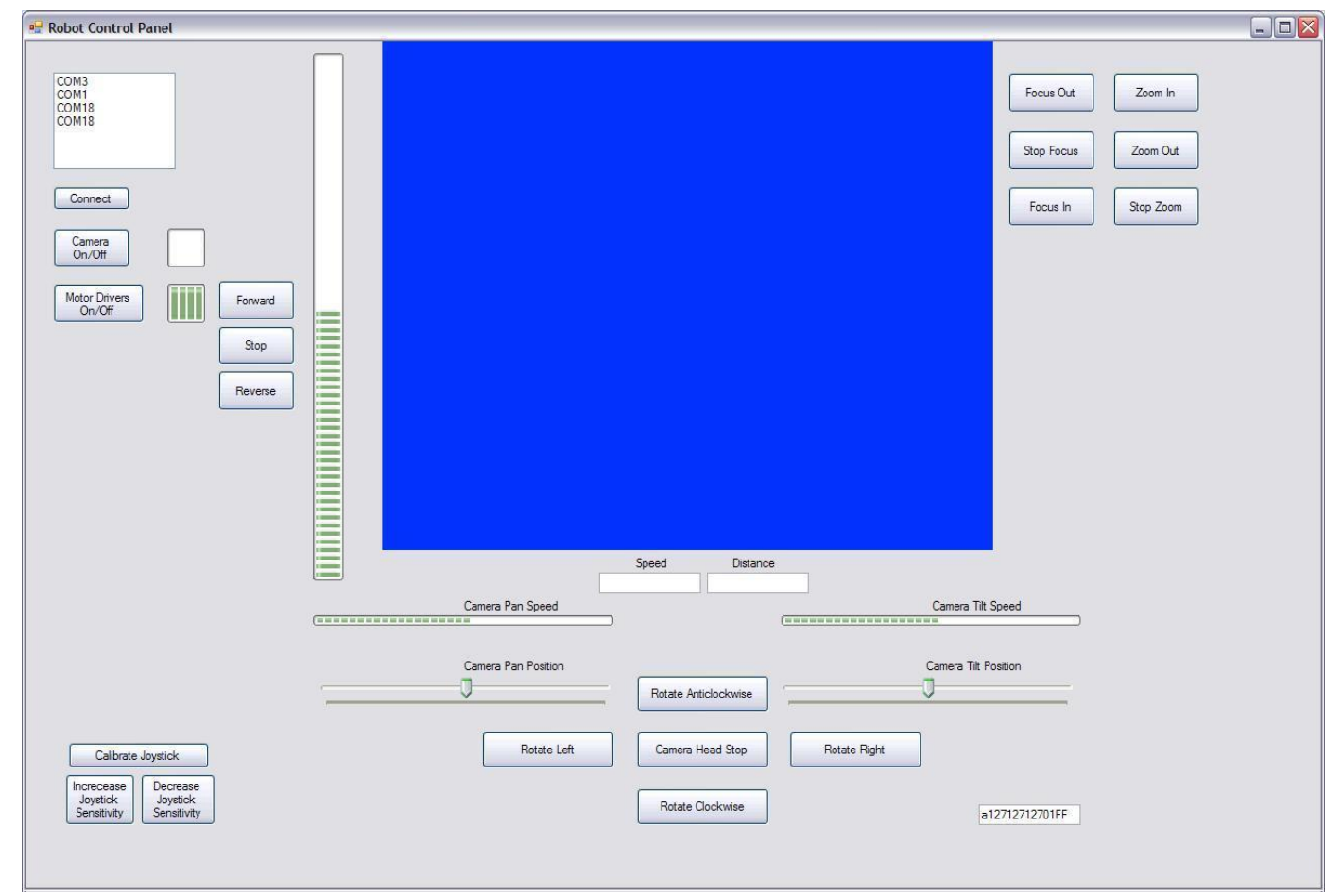

Figure 7.13: The fourth revision of the graphical user interface showing the new positioning of indicators and controls. 
The final modification made to the GUI is to rearrange the positioning of the buttons, indicators and video stream so that they are in the most suitable position for system control (Figure 7.13). The program is designed so that the eye is drawn to the most important parts of the program. As the human eye is drawn to movement, the video feed being displayed in the GUI is most likely to draw the attention of the user, hence the most significant control indicators and variables are placed in close proximity to this video feed. Figure 7.13 shows that the speed indicators, camera head position indicators and digital read-out of the robot's current speed and distance are closest to the video feed. Recalibration buttons for the joystick are the furthest away from the video as these are the least important controls when an inspection is taking place.

\subsubsection{Improvements Over Ristic Version}

When comparing the GUI designed in this project (Figure 7.13) with that designed by Ristic (Figure 2.16, reproduced below) the following improvements

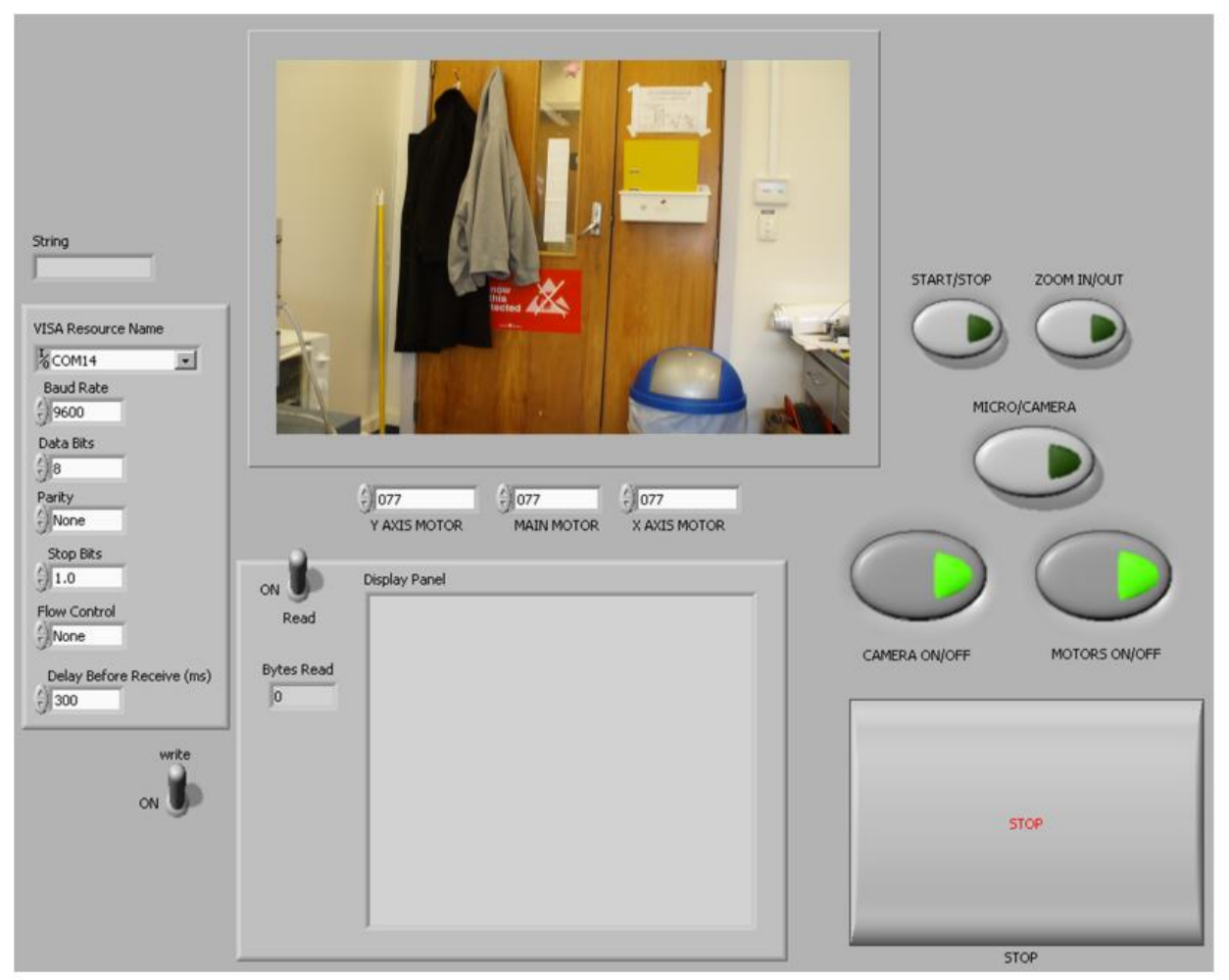

Figure 2.16: Mr Ristic's LabVIEW GUI 
can be noted.

The GUI designed is more user friendly with the adaptation of a joystick for control of the robot. The control also has an indicator for the speed of the robot as well as indicators for the speed and position of both the camera motors. This includes displaying current speeds in the form of graphical indicators and the overall speed and distance of the robot in the form of digital readouts.

The C\# GUI automatically connects to the robot and initiates the camera and motor drivers whereas the LabVIEW system does not. Due to this, the inclusion of a serial communication setup panel is not required, although the port that the robot is connected to is displayed.

The LabVIEW design uses large oval buttons for the camera control functions which are easy to see and click. Additionally the LabVIEW GUI has incremental inputs for all three motors which are not as easy to use. Both of these are redundant in the C\# version due to the use of a joystick for full control of the robot, although buttons for camera functions have been included as a backup if the joystick malfunctions. The zoom functions on the C\# GUI are setup to increment the zoom by one step per click whereas the LabVIEW setup uses continuous zoom until the stop button is clicked. This incrementation allows for more precise control of the camera hence the implementation.

Both GUIs have centrally located video windows, although the C\# GUI has a larger window and no additional border. The C\# GUI does not include a "recorded video display panel" as the LabVIEW GUI does since this was deemed to be a waste of space and computing resources, instead recording is handled "behind the scenes".

The final advantage of using C\# for the creation of the GUI is that it is freeware which allows AES to modify and upgrade the control software easily and freely. LabVIEW would cost the company USD $\$ 4,299$ to purchase if modifica- 
tions to the GUI were required (LabVIEW Professional would be required to produce .exe files [50]).

\subsection{Microcontroller code}

\subsubsection{Robot Code}

The code written to control the robot was initially started by Ristic; a full explanation can be found in his thesis [11]. This code included setting up 3 of the 5 timer modules as PWM outputs. These outputs relate to the camera drive motor, camera pan motor and camera tilt motor respectively. As stated in the Atmel AT89C51ac3 datasheet (see Appendix CD), these modules are hard-wired to pins 5 to 7 on port 1 . ASCII decoding and serial data packet communication is also programmed onto the microcontroller so that communication between the computer and robot via RS-232 was possible.

Slight modifications need to be made to this code to control the robot using the new C\# GUI. This includes adjusting the end character that the microcontroller looks for and setting limits and conditions for the motors. This is done to prevent the value sent to the motors from looping back to the other end of the register, hence causing the motor to run in the wrong direction. The final addition to this code is to add calibration constants for the motors. An offset is required for the robot to remain stationary at start-up as the motors are run via motor drivers (section 6.2.3) which are attached to the microcontroller via $\mathrm{RC}$ filters on the main control board (section 4.2.3). This offset is dependant on both the characteristics of the RC filter network and the motor driver. In using the Sabertooth $2 \times 5$ motor driver, the offset for the two camera motors is the same since the motor driver and RC filter between the microcontroller and the motors for both of these motors ares identical (within standard percentage 
errors). During testing, this value was determined to be 0x21 in hexadecimal, in other words the PWM duty cycle for the camera head motors needs to be reduced by 33 out of 255 to give an average voltage to the motor of $6 \mathrm{~V}$ (as these motors are driven by a $12 \mathrm{~V}$ signal). With the main drive motor the value was determined to be $0 \times 25$, or a duty cycle reduction of 37 out of 255 .

The final addition made to the robot code was to include an "if" statement to determine if the cable has gone slack. This can be done as the robot has an inbuilt sensor which sends a high pulse when the cable slackens. Therefore, the microcontroller is programmed to prevent the robot from reversing and damaging the cable when it is slack (Figure 7.14). With the motorised reel enabled, a warning will not need to be sent to the user as the reel will pick up the slack almost immediately as it will be programmed to engage and reverse when the robot is commanded to.

\subsubsection{Base Station Code}

As the base station is designed to control a motorised reel as well as read data from a rotary encoder mounted on the reel, this board requires a microcontroller (section 5.1). At present, the microcontroller on the base station is able to understand the control commands coming from the computer and to convert these into the signals required to drive a motorised reel in the desired direction.

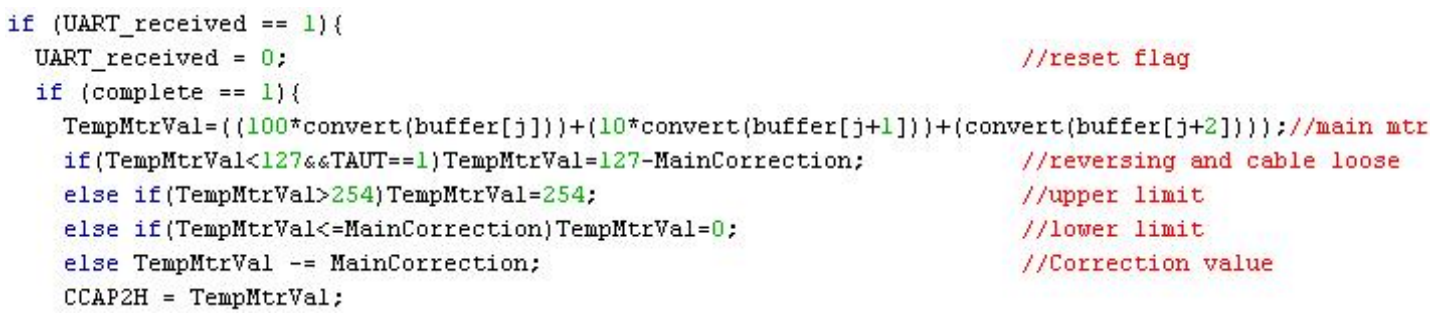

Figure 7.14: Part code for robot main motor control showing correction for motor driver and filter network as well as taut sensor and range conditions. 
The reel motor will be a single direction motor with free spooling capability requiring the code only to control the speed of the motor when the robot is reversing so that the cable is not damaged. Conditions for the reel have been programmed into the code for when the robot is moving. When moving in the forward direction the reel motor goes into a free spooling state which will either be handled using an electromagnetic clutch or internally by a winch motor. When the robot is slowing down the condition causes the reel motor to engage, providing some breaking to the reel and keeping the cable taut. When the robot is stopped, the reel is driven slightly in the reverse direction so that the cable is tight and if the robot then moves backwards the base station is able to respond instantly. Finally, when the robot is reversing, a slightly higher power is applied to the motor as the speed of the reel will be matched to the maximum speed of the robot.

The conditions have been inserted into the code but the actual values to send to the motor driver have not been finalised as both the motor and driver have not been sourced.

The base station is also designed to receive data from the robot and transmit the relevant parts of this data back to the PC, including the values of the camera rotation encoder and the cable sensor inside the robot. The base station will also provide a distance travelled and speed value back to the PC which is acquired from the rotary encoder attached to the reel. Although this is not the best method of acquiring the speed of the robot due to the inherent errors with measuring from an external source that has the possibility of expanding (the cable), it is the most practical solution for this robot.

Due to time constraints in this project which have prevented the sourcing and installation of the reel motor, the first revision of the base station micro controller code is written including all reel control functions, but is untested. Although the ASCII conversion has been directly copied from the robot code 
(section 7.2.1), further work on this code is needed and possible progressive steps are outlined in section 8.2.

\subsection{Code Summary}

A working GUI has been produced for control of the robotic unit. This GUI allows for a joystick input for control of the robot while displaying the video feed to the user. Position and speed indicators have been included in the interface to allow for easy visualisation of what the robot is doing. Additionally buffer regions for the three motors have been included as has serial data packet communication.

As a new GUI has been created, the microcontroller code on the robot had to be modified to allow for correct communication between the two.

A beginning to the base station code has been made with serial communication implemented and skeleton code established for reel motor control. 


\section{Chapter 8}

\section{Evaluation and Conclusion}

\subsection{Evaluation}

The objectives of this project were to redesign the PCBs required for this robotic system, source components and redesign the control software. The original specifications did not require work on the PCBs or software for the base station of the system nor the sourcing of cabling, seals and reel motors. This additional work was undertaken as an extension in order to better inform the hardware and software design for the robot.

The internal circuitry of the robot has been completed to a point where population and testing should result in a working robotic unit for this system, including a camera with resolution and clarity surpassing all researched systems. Additionally overheating of all internal components has been overcome by increasing the efficiency of the onboard regulators.

A GUI has been designed to be intuitive and user friendly by implementing a joystick for control of the robot and by locating all speed and distance indicators near the video window. Additionally recalibration of the joystick and reconnection to the robot is possible once the program is running. Buttons on 
the GUI are positioned so as to make the interface more intuitive and easier to use.

\subsubsection{Efficiency}

The efficiency of the regulators inside the robot determine how much heat is created. Overheating is a significant problem with this system as the robotic unit is airtight, hence there is no air flow to assist component cooling. A comparison between the linear regulators used on Ristic's design and the switch mode regulators used on these boards has been conducted for the main control (Figure 8.1) and camera (Figure 8.2) boards. The theoretical efficiency of the main control board displayed in Figure 8.1 is given at the maximum loading of the regulators. Input voltage indicators (12 V and $24 \mathrm{~V})$ have been included to illustrate where the comparison is most relevant. The LM2676 switch mode regulators on this board are $44 \%$ more efficient at converting $24 \mathrm{~V}$ to $12 \mathrm{~V}$ than linear regulators as shown on Figure 8.1 where the linear regulator is 50\% efficient and the switching regulator is $94 \%$ efficient. This figure also shows that

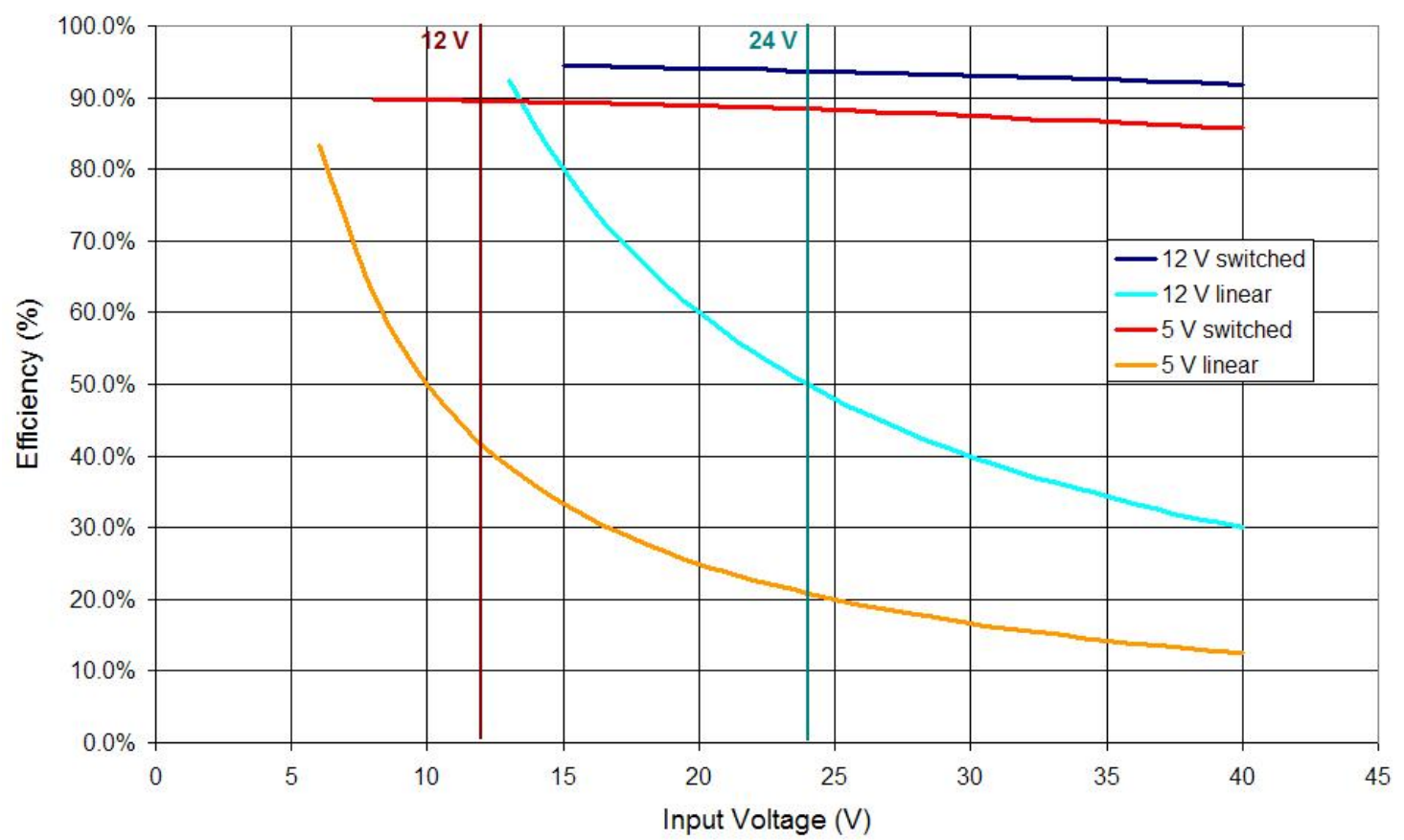

Figure 8.1: Efficiency of regulators on main control board 


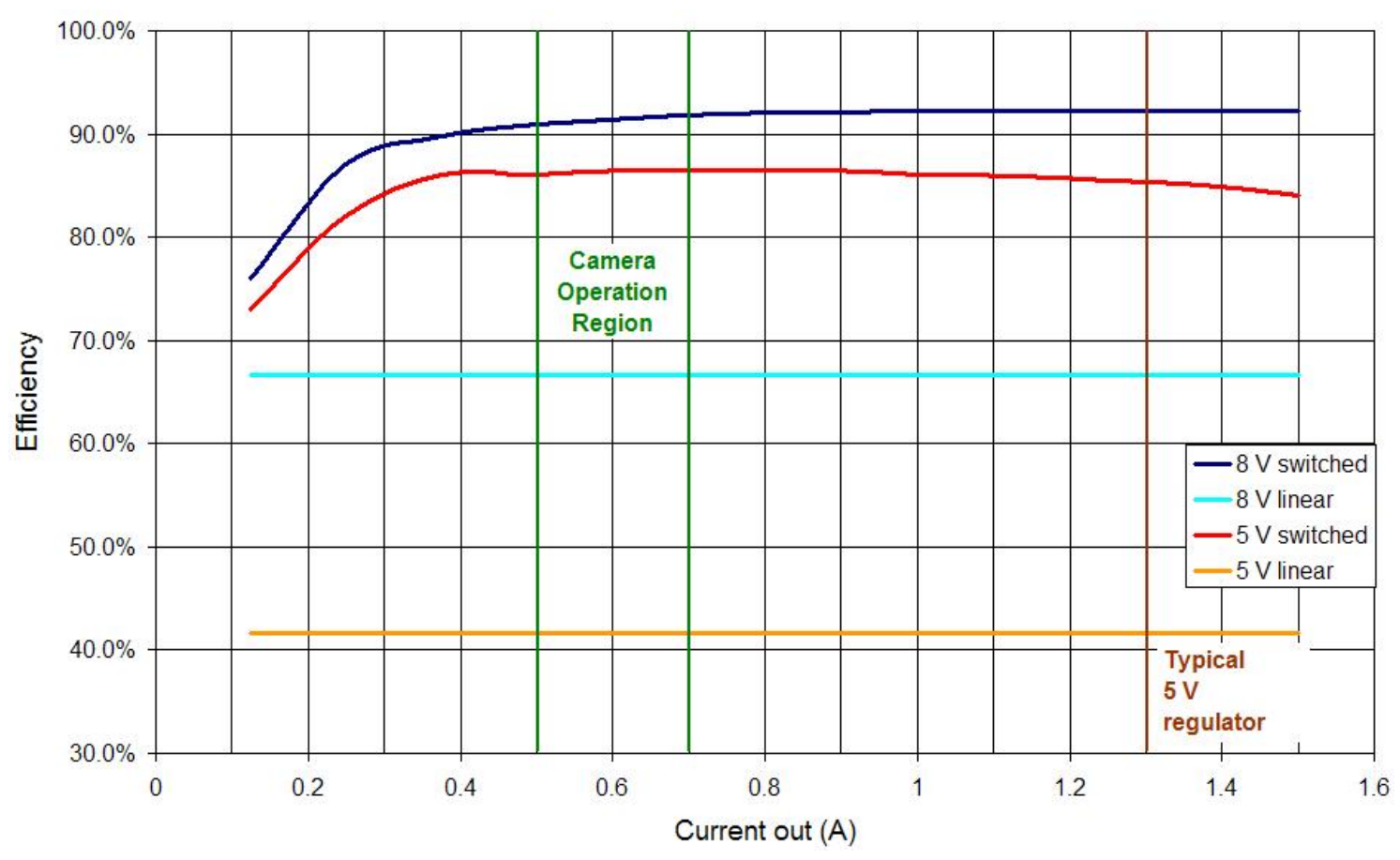

Figure 8.2: Efficiency of regulators on camera board

the $12 \mathrm{~V}$ to $5 \mathrm{~V}$ conversion is $48 \%$ more efficient as the switching and linear regulators are $90 \%$ and $42 \%$ efficient respectively.

The PT78st100 series regulators used on the camera control board are compared to their linear counterparts in Figure 8.2 at a constant voltage of $12 \mathrm{~V}$ due to both 5 and $8 \mathrm{~V}$ variants running off the same input voltage. Typically, the camera runs at between 0.5 and $0.7 \mathrm{~A}$ giving an efficiency improvement greater than $24 \%$ for the $8 \mathrm{~V}$ switch mode regulator. The $5 \mathrm{~V}$ converter typically draws $1.3 \mathrm{~A}$ and is approximately $43 \%$ more efficient than its linear counterpart.

\subsubsection{Review}

The final system designed in this project is a significant progression and advancement over the prior work of Ristic. The overheating of components inside of the robot has been mitigated and the internal circuitry has been redesigned to the point where once the boards are populated and the new camera is adapted the internals of the robot will be complete. Only slight modi- 


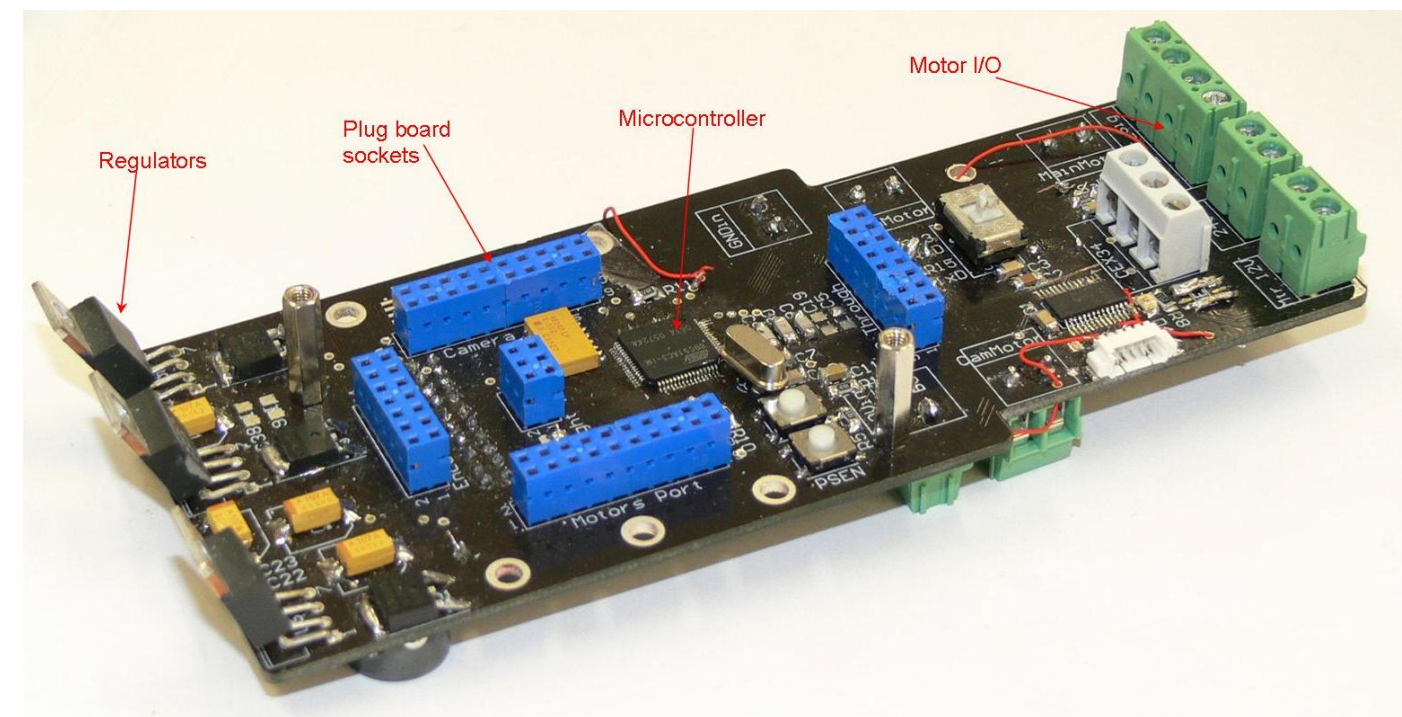

Figure 8.3: Photo of final populated version of the main control board (revision 3) displaying switch mode regulators, plug board sockets, microcontroller, programming switch and $\mathrm{I} / \mathrm{O}$ to and from motor drivers. Underside contains connection to power module, inductors and other motor terminals.

fications of the robot as a whole needs to be made, specifically replacing the seals and rebuilding the light holders (section 6.1) with a different material as acetal is not inert to chloride.

The main control board (Figure 8.3) is able to control the three motors inside the robot by utilising a PWM output from the microcontroller and passing it to a motor driver via a low pass filter. Additionally this board communicates with the base station via RS-485 data packet communication and is able to read all sensor inputs of the robot.

The plug board designed as an extension of the main control board (Figure 8.4) manages all wiring inside the base of the robot as well as allowing for easy connection and disconnection of components inside this base.

The power module board (Figure 8.5) used to protect the circuitry and Vicor converter from voltage spikes utilises two TVS diodes. Capacitors on this board reduce noise on both the input and output sides of the converter. Additionally this board creates an impedance loop to stabilise the voltage output of 


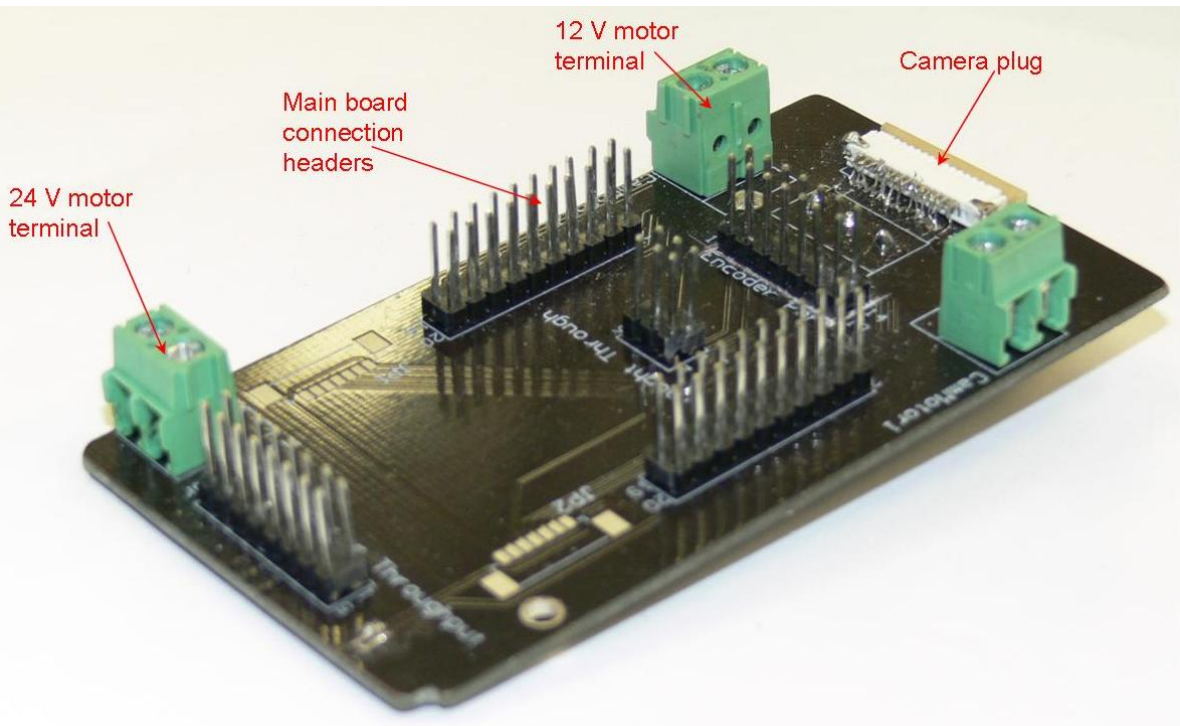

Figure 8.4: Photo of final populated version of plug board (revision 1), although this board has been manually cut to fit inside robot. The board contains $24 \mathrm{~V}$ motor terminal, connection headers to main board, $12 \mathrm{~V}$ motor terminals and camera board plug.

the converter for a larger range of loads.

The camera control board converts the RS-485 signal to RS-232 for the camera module as this is what the camera VISCA protocol runs on. Additionally this

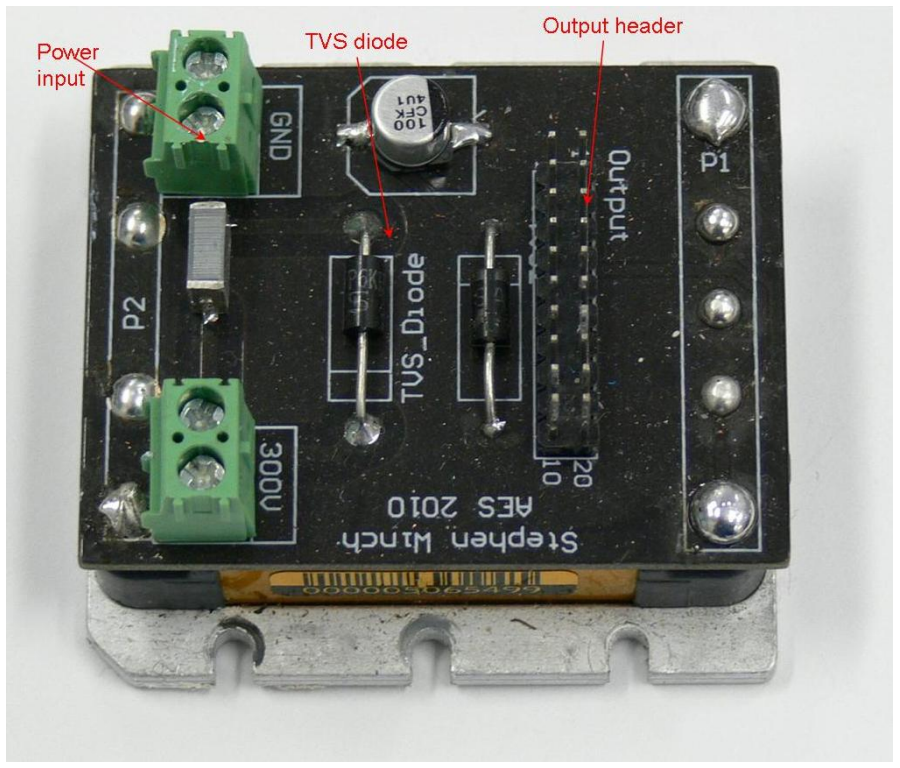

Figure 8.5: Photo of final populated version of power module board (revision 2), displays $300 \mathrm{~V}$ and Ground inputs, TVS diodes and output header to underside of main control board. Note the Vicor DC-DC module is attached underneath. 


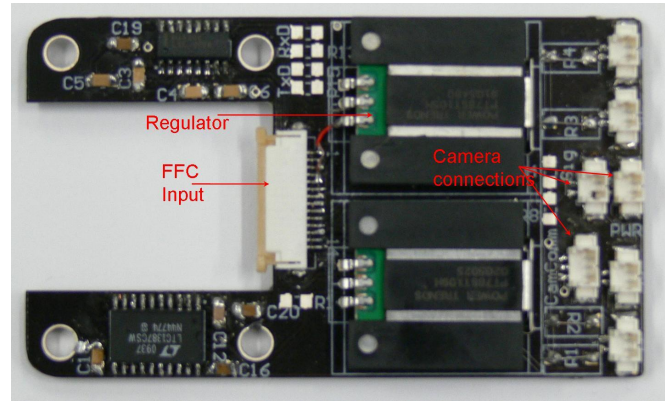

(a)

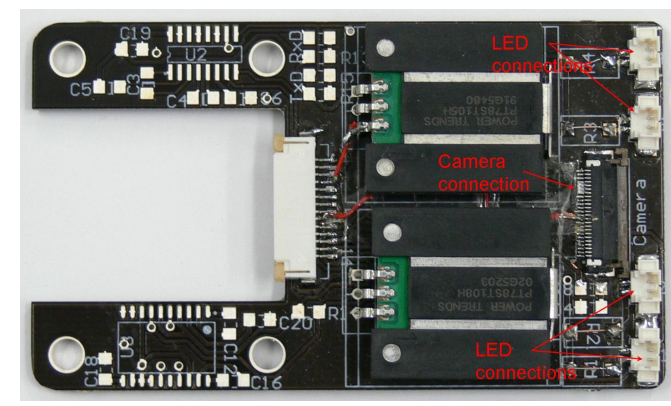

(b)

Figure 8.6: Photos of final versions of camera control boards populated (revision 2) The boards are for the Sony (a) EVI-371 and (b) FCB-H11 cameras. Note that full population is not complete due to time constraints.

board transforms the $12 \mathrm{~V}$ input to the required $8 \mathrm{~V}$ and $5 \mathrm{~V}$ for the camera and LEDs respectively. Two versions of this board exist for the older EVI-371 camera and the new FCB-H11 (Figures 8.6(a) and 8.6(b) respectively).

A new camera module has been acquired from Sony New Zealand and once this camera module becomes fully operational the robot will surpass all inspection robots researched for video resolution and clarity.

As the camera head is a rotating component connected to the base of the robot, the wires connecting the two have to be taken into account when the camera head is rotated otherwise over rotation of the camera head in a single direction can cause the wires to snap. Both software based and hardware based solutions were considered and implemented. The software limitation 'prevents the camera head from rotating more than $\pm 370^{\circ}$ by reading the value from the shaft encoder and recording the position of the camera head and displaying it to the user. $370^{\circ}$ is used as this allows for overlapping of the positive and negative rotation which allows for a full $360^{\circ}$ view of the pipe without the possibility of blank spots.

The hardware backup protection is a screw on the shaft of the robotic head which comes into contact with a limiting rod which is able to move side to side. The distance that the rod is able to move can be adjusted by reducing the size 


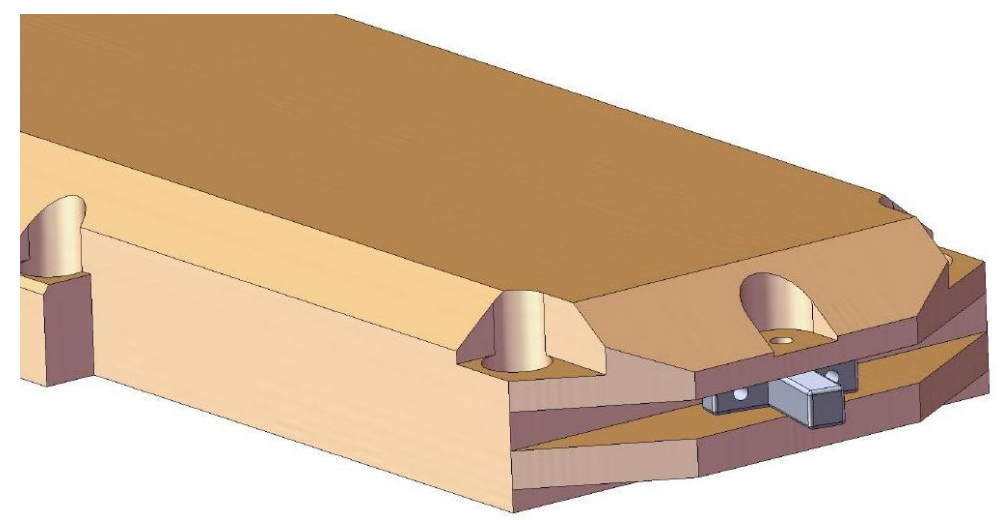

Figure 8.7: Robot lid with over rotation prevention rod.

of the screws extruding out each side. These screws stop the rod when they come into contact with the lid of the robot (Figure 8.7). By implementing both of these methods the integrity and reliability of the cable and wires connecting the base of the robot to the head is maintained.

The base station (Figure 8.8) of this system has been designed to understand commands from the user and to control a motor in the desired way, thus allowing for a fully autonomous motorised reel. The type of motor required to drive the reel has been narrowed down to an AC motor or a high voltage DC motor $(300 \mathrm{~V})$ with a single phase AC variant being the preferred option (section 6.2.7).

The base station has also been setup to be the junction between transmission from the robot and the computer. Data from the reel encoder can be added to the data stream providing the computer with the information on the distance that the robot has travelled. Additionally the board is able to control a motorised reel via a commercial motor driver or the custom made $130 \mathrm{~V} 110 \mathrm{~A}$ motor driver (Figure 8.9).

The graphical user interface designed in C\# allows for the easy control of the robot via a joystick. The GUI was designed to be robust and reliable by testing multiple scenarios and fixing any bugs that were found in these tests. Scenarios tested include prematurely closing the program, cancelling the saving 
of the video file, clicking one of the motor or camera control buttons when the joystick was connected as these operations conflict with each other; and not having the robot plugged in and turned on when the program is started. For the first and last case stated, the program will safely exit and will require restarting if control is required. In the last case, a message box will appear asking the user if they have connected the robot before the program closes. For the second case, the default is for the joystick to take priority over the buttons on the GUI as this is the more user friendly device to use. However GUI camera zoom and focus buttons still work in an incremental fashion where a single click will cause the camera to increase or decrease the zoom or focus by one step. Previously the code was written so that the camera would continue the operation until stopped.

This control interface currently has the option of video recording as well as continuously displaying the video to the user. The video recording has been

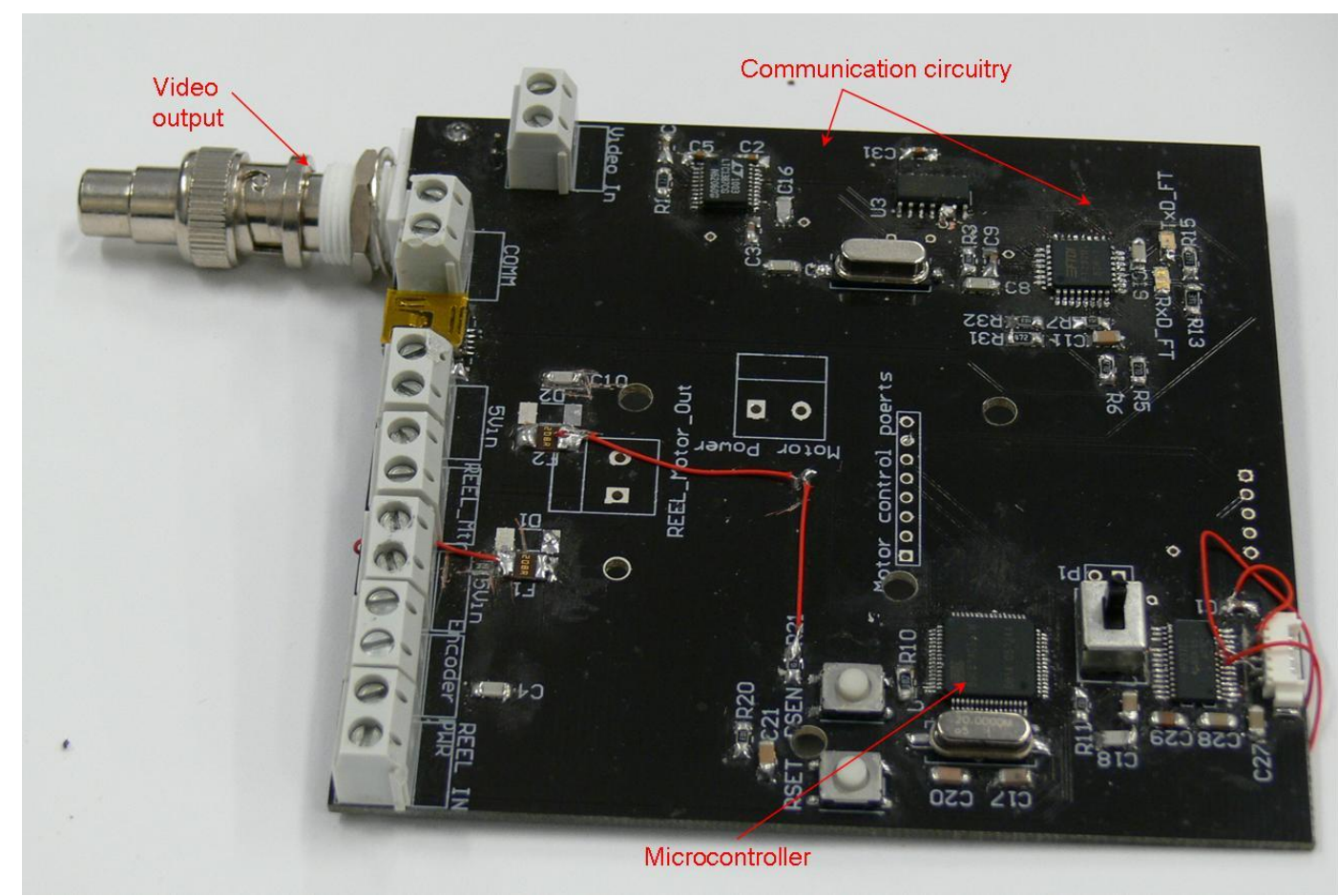

Figure 8.8: Photo of final populated version of base station board (revision 2), displays the I/Os including the video output,USB to RS-232 and RS-485 circuitry and microcontroller and reprogramming circuitry. 


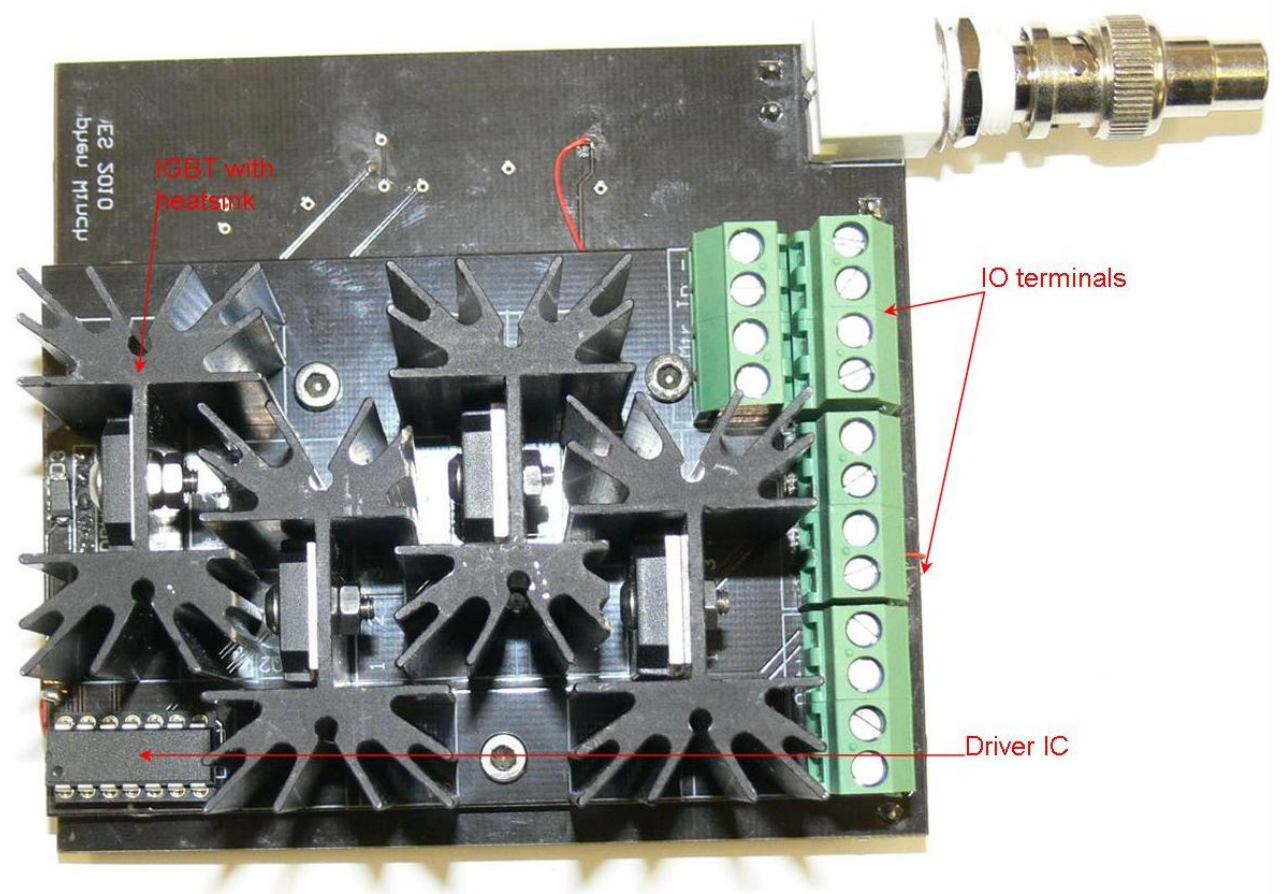

Figure 8.9: Photo of final populated version of the motor driver (revision 1), displays the 4 IGBTs and heat sinks used as well as the I/O headers and driver IC on a DIP socket. Note that the driver is attached directly to the underside of the base station board.

implemented with the option of selecting between several compression algorithms so that file size can be reduced without significantly reducing the picture quality. However, some of these algorithms can cause the video to lag and thus not display in real time (section 7.1.4). Options to overcome this include multi-thread programming or using an external recording device.

Cabling options for the system have been located at Tyco electronics, General Cable, Lapp Group and Falmat, of which Tyco provided the most information and is likely to be able to supply the required cable.

High end DC micro motors have been located at Faulhaber and contacts have been made with their local supplier Erntec[38], and so an upgrading of the motors inside both this robot and the larger version can easily be conducted by AES in the near-future.

The light holders designed for this system are functional, giving a watertight 
Table 8.1: Project outcomes and prospects versus ideal outcomes.

\begin{tabular}{|c|c|c|c|c|}
\hline & & \multirow[b]{2}{*}{ Ideal } & \multicolumn{2}{|c|}{ Project } \\
\hline & & & Implemented & Possible \\
\hline \multirow{2}{*}{$\begin{array}{l}\text { Pipe } \\
\text { diameter } \\
\text { possible }\end{array}$} & Minimum & $100 \mathrm{~mm}$ & $150 \mathrm{~mm}^{[1]}$ & $150 \mathrm{~mm}^{[1]}$ \\
\hline & Maximum & $1.5 \mathrm{~m}$ & $1 \mathrm{~m}^{[1]}$ & $1 \mathrm{~m}^{[1]}$ \\
\hline \multirow[t]{5}{*}{ Camera } & Resolution & $450^{+}$TVL & $450 \mathrm{TVL}^{[2]}$ & 2MP HD \\
\hline & $\begin{array}{l}\text { Zoom } \\
\text { (Optical) }\end{array}$ & $12 \times$ & $12 \times$ & $10 \times^{[3]}$ \\
\hline & $\begin{array}{l}\text { Zoom } \\
\text { (Digital) }\end{array}$ & $10 \times$ & $10 \times$ & $12 \times^{[3]}$ \\
\hline & Rotation & $360^{\circ}$ & $370^{\circ}[1]$ & $370^{\circ[1]}$ \\
\hline & Pan & $\begin{array}{l}\text { continuous } \\
360^{\circ} \\
\text { continuous }\end{array}$ & $270^{\circ[1]}$ & $270^{\circ[1]}$ \\
\hline \multirow[t]{6}{*}{ Robotic Unit } & Casing & Inert & Mostly Inert $^{[4]}$ & Inert \\
\hline & & Watertight & Exposed & Watertight \\
\hline & & Solid & Solid & Solid \\
\hline & Other & Modular & Singular Unit & Two Units ${ }^{[5]}$ \\
\hline & & Mil. Spec. & Mil Spec. & Mil Spec. \\
\hline & & Connector & & \\
\hline \multirow{7}{*}{$\begin{array}{l}\text { Control } \\
\text { System }\end{array}$} & Display & In-Built & In-Built $^{[6]}$ & In-Built $^{[6]}$ \\
\hline & Keys & Programmable & $\begin{array}{l}\text { Pre- } \\
\text { programmed }\end{array}$ & Either \\
\hline & Control & Joystick & Joystick & Joystick \\
\hline & Other & Watertight & Exposed & Watertight \\
\hline & & Ergonomic & Ergonomic & Ergonomic \\
\hline & & Intuitive & Mostly & Intuitive \\
\hline & & & Intuitive $^{[7]}$ & \\
\hline \multirow[t]{4}{*}{ Reel } & Type & Motorised & $\mathrm{N} / \mathrm{A}$ & Motorised \\
\hline & & Portable & $\mathrm{N} / \mathrm{A}$ & Mostly Portable \\
\hline & & Automated & $\mathrm{N} / \mathrm{A}$ & Automated \\
\hline & Cable size & $300 \mathrm{~m}$ & $200 \mathrm{~m}$ & $300 \mathrm{~m}$ \\
\hline
\end{tabular}

[1] Physical limitations of robotic unit chassis

[2] $450 \mathrm{TVL}$ is equivalent to 270,000 pixels in 4:3 aspect ratio

[3] Sony FCB-H11 camera provides less optical zoom but higher resolution video

${ }^{[4]}$ All but the light holder (section 6.1) are inert, problem explained in section 8.2.1

[5] Possibility of using same cabling and control system for larger robot one this is fully implemented

${ }^{[6]}$ As the control system includes a laptop it comes with an inbuilt screen

${ }^{[7]}$ Fully intuitively cannot be stated due to limited testing 
seal as well as allowing for easy replacement of the lights if one dies.

When comparing the system implemented to the specifications for the ideal system outlined in Table 2.2 in section 2.1.4, the modifications made allow the robot to compete with current industrial units. The old camera in the robot is equal to a majority of current cameras and the newly acquired camera surpasses all of these even without the HD mode implemented. Table 8.1 is a comparison between all factors outlined in the ideal system (section 2.1.4), the current system and the possibilities of this system.

\subsection{Future Work}

\subsubsection{Robot}

If a decision to use the high definition output of the Sony FCB-H11 camera is made, a modification will be required to implement three dual line video signals instead of the one dual line video output. Once video output is currently used for transmission of a VBS (Video, Blank, Sync) signal and ground line for the coaxial cable shielding. To transmit the three video signals a modification would be required on both the plug board (section 4.3) and the camera board (section 4.1) so that the video signal can be sent from the camera to the base station. This would require replacing the currently connected 14 line FFC (Flat Flexible Cable) to accommodate for the extra video lines. To do this a cable with line spacing of less than $0.8 \mathrm{~mm}$ would be required to fit inside the robot (the current cable has $1 \mathrm{~mm}$ line spacing whereas the camera cable has $0.5 \mathrm{~mm}$ pin spacing).

The camera head has been prevented from rotating over $370^{\circ}$ by both the GUI control system and a hardware prevention. For the GUI prevention to work the distance that the camera has rotated needs to be sent from the robot to 


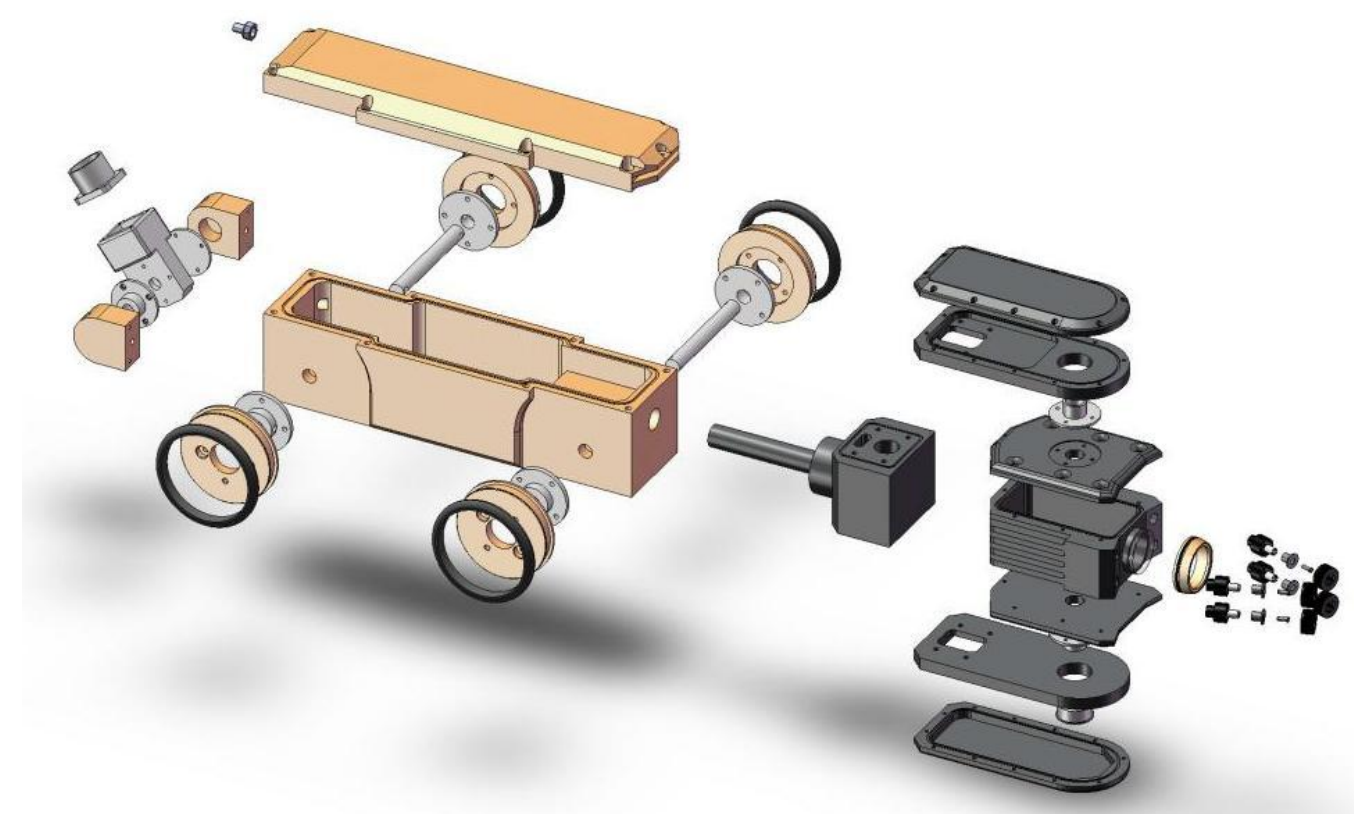

Figure 8.10: exploded view of robotic unit showing extent of seal replacement required

the computer. This has not yet been implemented due to time constraints but would require the code on the robot microcontroller to be modified so that data from the camera head encoder can be recorded. Transmitting this distance of rotation back to the computer would allow the software to prevent over rotation of the camera head whist displaying the distance and direction of rotation to the user so that the orientation of the camera in the circular, uniform pipe is know.

The distance of rotation would then be transmitted back to the base station so that the user knows how far the head has rotated and if they are required to rotate in the other direction.

The final modifications that need to be made to the robotic unit are to get the light holders rebuilt as the current material is acetal. Acetal is chemically inert to almost everything except chloride, which happens to be one of the components that will be found inside of sewage pipes as it is used in many house-hold cleaners. The other components on the robot that need to be replaced are the 
seals. Due to the age of the system these seals are broken and ineffective which will allow water to penetrate into the system. Two options exist for this seal replacement, the first is to cut o-rings and fit them to the robot. This method is the cheapest option but probably also the least reliable. The other option is to get seals custom made for the robot. One possibility is Petone based Pacific Seals Limited [51] which supply standard seals as well as custom making seals for any requirement. The extent of the seal replacement required can be seen clearly in Figure 8.10.

\subsubsection{Base Station}

The first task in completion of the base station for this system is to decide which motor would be most applicable, whether this should be a $300 \mathrm{~V}$ motor or a single phase $240 \mathrm{~V}$ AC motor is currently unknown (section 6.2.7). Once this selection has been made, the type of controller required can then be determined.

Whether the base station requires an additional component in the form of a hardware video recording device has yet to be decided. Real time video compression is required due to the length of video required to be recorded and the size of each frame in that video (section 7.1.4). Compressing using the computer requires a significant amount of processing power which a dual core machine can only provide with the use of both cores. Thus one option is that the entire control GUI needs to be separated into sections such that a multithread program can be created.

Another option for recording the video would be to use an external hard drive video recorder. This option would allow the user to interact with the robot without having to worry about the recording of the video. This would allow for switching of hard drives so that the field technicians are able to hand a days recording to the analysts. This option is the one that will be pursued due to 
the ease of implementation, repair and human interfacing.

The addition of a hardware recorder should only affect the configuration and location of components inside the base station and should not directly affect the base station control board. The components that would possibly have to be moved include the yet to be sourced motor driver and the transformer which takes the AC input and converts this to 300,15 and 5 V DC.

If this system is to be fully portable, the transformer might need to be replaced as currently a separate isolating transformer is required as well as the base station itself. Thus a new transformer with built-in isolation would be sourced with the size and weight of the unit being significant factors on deciding which unit to use.

The base station code written so far has the ability to understand all commands sent by the computer and has skeleton code implemented to control the motorised reel. This code has not been finalised as the motor or controller for the reel has not yet been determined. Skeleton code has been written to understand each action of the robot and to control the reel in the desired manner. This is done using serial data packet decryption to understand the commands sent to the robot and a timer module setup as a PWM to control the motor. Another timer module has been setup in capture mode so that the encoder attached to the reel can be read. Values for converting the encoder signal into speeds and distances have not been included as these differ from encoder to encoder and are dependant on the clock speed of the microcontroller as well (equation 8.1[52]).

$$
R P M=\frac{(\text { seconds } / \text { minute }) *(\text { counts } / \text { second })}{(\text { holes } / \text { revolution }) *(\text { counts } / \text { hole })}
$$

In this equation the counts/second variable is the speed in which the applicable timer of the microcontroller counts, the holes/revolution variable is defined by 
the physical layout of the encoder used and the counts/hole variable is determined by the incoming signal causing a timer interrupt. By taking the current time and the time of the last interrupt it is possible to determine the speed the robot is travelling and therefore the distance that it has travelled.

The microcontroller also needs to send control signals to a motor to control the reel in the desired direction as outlined in section 7.2.2. Additionally it must continue to perform the task of communicating with the computer.

\subsubsection{GUI}

One possible upgrade to the GUI would be to have calibration buttons for each of the motors, as the motors do have offset values due to the filter circuitry. These buttons would be used at the start-up of the program to re-calibrate the robot to a steady state, thus stopping the motors from moving when the robot is not meant to. All that would be required inside the control system would be some variable stored that is added to the motor value before being transmitted to the robot.

Once the base station has been fully implemented and is designed to transmit back to the computer, receiving of this signal needs to be handled so that the data can be displayed to the user.

A method of resetting the camera position to its original state would be a useful addition. The rotation of the camera can easily be reset using the encoder attached to the respective motor but the pan of the camera cannot due to the motor lacking an encoder. The average signal sent to the motor cannot be used as the frame of the robot stops the camera head from moving while power is still being applied. Therefore, the options of adding an encoder to this motor or setting the program to prompt the user to reset this motor on closure exist. 


\subsubsection{Reel design}

An option exists to employ $200 \mathrm{~m}$ of cable instead of the $300 \mathrm{~m}$ cable desired by AES, but this will significantly reduce the length of piping that can be inspected at a single time, and hence significantly increase the time taken for the technicians to inspect $1 \mathrm{~km}$ of piping.

The second option is to expand the size of the reel by increasing the height of the frame and the size of the side disks. An increase of $0.1 \mathrm{~m}$ in diameter will allow for an additional 148 to 175 metres of cable to be added depending on the thickness of the cable (calculations are for $10 \mathrm{~mm}$ and $8 \mathrm{~mm}$ cable respectively). Currently the reel is made from steel. If an aluminium frame is used instead, the overall weight will be halved which would allow for the technicians to still be able to carry the system to the desired locations.

The final option is to purchase a new reel which could possibly be motorised. Motorised reels that have been located and are able to be used for this application are vehicle mounted and hence unable to be carried. Therefore a custom motorisation might be preferable, where the reel and motor can be separated from transport.

\subsection{Summary}

Overall the aims of this project have been met, with significant progress being made towards having a fully operation pipe inspection robot. Problems such as heat dissipation, user interfacing, and size constraints have been overcome, and solutions to problems such as sourcing of motor and cabling replacements have been found.

Contradictions between the desires of AES and the reality of the system have been found and compromise solutions have resulted. Once the current revi- 
sions of the internal boards have been populated a fully functional robotic unit will be given to AES. 



\section{References}

[1] Pobar, B. Private correspondence. At Victoria University, July 2009.

[2] Christchurch City Council. http://www.ccc.govt.nz. Last accessed $20 / 06 / 10$.

[3] Wellington City Council - New Zealand. http://www.wellington. govt.nz. Last accessed 20/06/10.

[4] Manukau Water: Manukau Water. http://manukauwater.co.nz. Last accessed 20/06/10.

[5] Radiodetection - Welcome! http://www.pearpoint.com/. Last accessed 18/06/10.

[6] IBAK Helmut Hunger GmbH \& Co. KG: Home. http://www. ibak. de/. Last accessed 18/06/10.

[7] iPEK Home. http://www. ipek . at/. Last accessed 18/06/10.

[8] Envirosight: Specialists in Pipe Inspection and Rehab. http://www. envirosight.com/. Last accessed 18/06/10.

[9] OPTIMESS Gesellschaft für Optoelektronische Inspektions - und Messtechnik mbH. CCTV Unit MCA150.

[10] HowStuffWorks - Learn How Everything Works! http://www. 
howstuffworks. com. Last accessed 22/06/10.

[11] Ristic, A. Iterative Approach on Redesigning and Construction of a Pipe Inspection Robot. Masters Thesis, Victoria University of Wellington (2009).

[12] Newton, H. Newton's Telecom Dictonary (CMP Books, New York, 2001).

[13] Serial Cable Length Limits (RS232, V.35, RS530, RS422/449), SCSI Cable Length Limits, Parallel Cable Length Limits. http://www . connectworld. net/cable-length.html. Last accessed 08/07/10.

[14] RS485, specifications and in depth tutorial. http://www . lammertbies.nl/comm/info/RS-485.html. Last accessed 08/07/10.

[15] EIA485 Bus Interface Description, RS485. http://www . interfacebus.com/Design_Connector_RS485.html. Last accessed 08/07/10.

[16] RS485 Tech info. http://www.arcelect.com/rs485_info_ tutorial.htm. Last accessed 08/07/10.

[17] QIMAGING. FireWire® vs. USB 2.0 (2006). http: / / www . qimaging . com/support/pdfs/firewire_usb_technote.pdf, last accessed 08/07/10.

[18] USB.org. Univeral Serial Bus Specification. (C) Compaq Computer Corporation, Hewlett-Packard Company, Intel Corporation, Lucent Technologies Inc, Microsoft Corporation, NEC Corporation, Koninklijke Philips Electronics N.V. (2000).

[19] Source of plastic optical fiber and cable - Industrial Fiber Optics, Inc. http://i-fiberoptics.com/index.php. Last accessed 08/07/10.

[20] Meyers, R. A. Encyclopedia of Telecommunications (Academic Press Inc, Cal- 
ifornia, 1989).

[21] Horak, R. Telecommunications and Data Communications Handbook (WileyInterscience, New Jersey, 2007).

[22] YUV, YCbCr, YPbPr colour spaces. http://discoverybiz.net/ enu0/faq/faq_YUV_YCbCr_YPbPr.htm. Last accessed 22/06/10.

[23] Hamilton, E. JPEG File Interchange Format. C-Cube Microsystems, 1778 McCarthy Blvd; Milpitas, CA 95035, 1.02 edn. (2000). http://www . jpeg.org/public/jfif.pdf, last accessed 21/06/10.

[24] Colantoni, P. Color Space Transformations (2004). http: / / www . couleur. org/index.php?page=transformations, last accessed 22/06/10.

[25] Farnell New Zealand - world-leading distributor of electronic and maintenance, repair and operations products. http://nz.farnell.com/. Last accessed 23/06/10.

[26] Vishay - manufacturer of discrete semiconductors and passive components. http: //www . vishay . com. Last accessed 02/12/09.

[27] Howard, W. Handbook of Electronics Tables and Formulas (Sams, 1986), 6th edn.

[28] Powerbox Australia. http://www.powerbox.co.nz. Last accessed 05/08/09.

[29] Vicor Power — DC-DC Converter Modules, Configurable Power Supplies, and Systems. http://cdn.vicorpower.com/. Last accessed 19/01/10.

[30] DigiKey Corp. - Electronic Components Distributor - New Zealand Home Page:. http: //www. digikey. co.nz/. Last accessed 23/06/10. 
[31] Robot Power On-line Catalog. http://www.robotpower.com/ catalog/. Last accessed 28/07/10.

[32] Sony Product Detail Page - FCBH11. https://pro.sony.com/bbsc/ ssr/cat-industrialcameras/cat-block/product-FCBH11/. Last accessed 02/03/10.

[33] Dimension Engineering - R/C, Power Electronics, Sensors. http:// www. dimensionengineering.com/. Last accessed 15/11/09.

[34] RS New Zealand - World Leading Distributor of Electronics, Electromechanical and Industrial Components:. http://newzealand. rs-online.com/web/. Last accessed 23/06/10.

[35] Jaycar Electronics - Better. More Technical. http://www - jaycar.co. $\mathrm{nz} /$. Last accessed 23/06/10.

[36] Active Components :: 1,000,000+ Electronic Components - Authorized Mouser Reseller:. http://www.mouser.co.nz/. Last accessed 23/06/10.

[37] FAULHABER Miniature Drive Systems - FAULHABER Group Brushless Motors and DC Motors:. http: / / www . faulhaber. com/. Last accessed 15/01/10.

[38] ERNTEC : Home:. http: / / erntec . net. Last accessed 22/02/10.

[39] General Cable NZ Limited:. http://www.generalcable.co.nz/. Last accessed 13/06/10.

[40] FALMAT Wire and Cable - Custom Cable Manufacturer. http: / / www . falmat.com/. Last accessed 18/06/10.

[41] Lapp Group :: Home. http://www.lappgroup.com/2/en/home/ index.html. Last accessed 13/06/10. 
[42] Tyco Electronics - Electronic Components, Connectors \& Network Solutions:. http://www.tycoelectronics.com/. Last accessed 13/06/10.

[43] Lammers, M. Incident - Cabling enquiry. Email. Initiated : 15/06/10; Last Responce: 16/06/10.

[44] EMTEQ - Aviation and defence products, engineering and support. http: //www. emteq. com/. Last accessed 13/06/10.

[45] Choosing between C\# and VB.NET. http://www. dnjonline.com/ article.aspx?ID=mar05_vbvscsharp. Last accessed 05/08/09.

[46] Your Development Resource - CodeProject. http://www . codepro ject. com. Last accessed 27/05/10.

[47] Sony Corporation. VISCA $A^{T M}$ RS-232C Control Protocol.

[48] Logitech Extreme 3D Pro Joystick (Silver/Black). http://ucables. com/ref/LOGITECH-EXTREME-3D-P-R53253. Last accessed 05/07/10.

[49] MSDN Home Page (United Kingdom - English). http://social. msdn.microsoft. com. Last accessed 02/04/10.

[50] NI LabVIEW - Improving the Productivity of Engineers and Scientists. http://www.ni.com/labview/. Last accessed 9/08/10.

[51] Welcome - Pacific Seals. http://www.pacificseals.co.nz/. Last accessed 25/04/10.

[52] Jongenelen, A. \& Carnegie, D. ECEN301 Laboritory Manual. Victoria University of Wellington, 5th edn. (2010). 



\section{Appendix : CD Contents}

$C D$ is attached to inside rear cover

Thesis.pdf Thesis.tex

\section{Code}

BaseStation.c

C file compiled to a hex file to programme onto base station microcontroller, this code is designed to control a motorised reel depending on the speed and direction of the robot. It also is designed to read the rotary encoder that outputs the distance travelled by the robot.

PipeBot.c This code is designed to control the robotic unit including deciphering the data packet sent to it from the computer via the base station board, controlling the 3 motors inside the robot, the camera and LEDs mounted on and in the robot.

RobotControl.zip Zip file containing robot control GUI designed for video capture and robot control via RS-485 with a joystick. 


\section{Documentation}

\section{Cameras}

EV370_Command_List.pdf $\quad$ VISCA $^{\mathrm{TM}}$ commands for Sony EVI-371 camera.

EVI_371_MANUAL.pdf Old Sony EVI-371 camera block module datasheet.

FCB-H11 tech manual.pdf New Sony FCB-H11 camera block module technical datasheet.

FCB-H11.pdf New Sony FCB-H11 Basic explanation pamphlet.

ViscaProtocol.pdf Physical setup of Visca protocol

\section{Components}

DC-DC converter.pdf

pt78st100.pdf

Sabertooth2x5.pdf

SyRen10-20.pdf
Vicor $250 \mathrm{~W}, 300 \mathrm{~V}$ to $24 \mathrm{~V}$ DC to DC transformer used inside the robot.

$5 \mathrm{~V}$ and $8 \mathrm{~V}$ integrated switching regulators used on the camera control board datasheet. Sabertooth $2 \times 5$ motor driver datasheet. This dual 12 V 5 A motor driver and its datasheet was acquired from Dimension Engineering (www.dimensionengineering.com).

The SysRen 1024 V 10 A motor driver was also acquired from Dimension Engineering.

\section{ICs}
ACPL-224.pdf
Opto-isolator chip. 
at89c51ac3.pdf

DA108S1.pdf

FDS4935BZ.pdf

FT232BL.pdf

IXTQ130N10T

LM324.pdf

LM339.pdf

LM2676.pdf

LTC1387.pdf

MAX202CPE.pdf

TC4469CPD.pdf
8 bit microcontroller full datasheet.

Quad protection diode pack.

Dual P_MOSFET.

USB to TTL chip.

130 A $100 \mathrm{~V}$ power MOSFET.

Quad operational amplifier.

Quad low power comparator.

Switch mode regulator IC.

RS232/RS485 Multiprotocol Transceiver.

RS-232 Transceiver.

CMOS quad motor driver logic chip.

\section{Other}

2RM360 encoder.pdf

at_c51instruction manual.pdf

Cam Tilt Gearbox.pdf

Cam Tilt Motor.pdf

Gerber and drill export.pdf

KFW150 Service manual.pdf KPY43A pressure sensor.pdf USB2.zip
The datasheet for the camera tilt head encoder.

Microcontroller instruction set.

Faulharber gearbox from ErnTec for use with the camera tilt motor if replacement is required.

12 V Faulharber motor acquired from ErnTec in Australia (http : //www.erntec.NET/).

Advanced Circuits New Zealand export specifications for PCB creation.

Original service manual for robot being repaired.

Pressure sensor found inside robot.

Documentation on USB2.0 standard. 


\section{Robotic Systems}

IBAK_Argus_eng.pdf

IBAK_Kameras_eng.pdf

IBAK_Kanal_TV_e.pdf

IBAK_Kanal_TV_eng.pdf

IBAK_KRA_85_eng.doc

IBAK_KRA_85_s_d.pdf

IBAK_KRA_d.pdf

IBAK_Lisy_1_2_eng.pdf

iPek Rovver.pdf

P350_FlexitraxBroV5_en.pdf

pathfinder2.pdf

Pearpoint_Catalogue.pdf

PTMianline.pdf

Rovver100.pdf

Rovver125.pdf

Rovver225brochure.pdf

XPRESSCrawlLitMaster.pdf
IBAK Argus system details.

IBAK Camera details.

IBAK System diagram overview.

IBAK detailed system overview.

Translated IBAK KRA 85 details.

IBAK KRA 85 details (in German).

IBAK KRA 65 details (in German).

IBAK Lisy attachment details.

Details of Rovver units supplied by iPek.

PearPoint portable system.

Another pipe inspection system.

PearPoint main units catalogue.

Another pipe inspection system.

Rovver100 details (Envirosight).

Rovver125 details (Envirosight).

Rovver225 details (Envirosight).

Another pipe inspection system.

\section{Results and Calculations}

Joystick to motor driver trendlines 136 max.xls

Joystick to motor driver trendlines at 255 bit.xls

Length Of Cable On Reel.xls

Logitech Joystick Pipe Inspection Robot Controls.doc

Main motor characteristics.xls

Motors for reel.xls

Regulators efficiency.xls

Uncompressed Video.xls 
Video Compression Comparison.xls

Winches.xls

\section{Schematics and PCB layouts}

Note that PCB layouts have also been included as the positioning of components was an intrinsic part of this project.

\section{Base Station Board}

BaseStationBoardMyRev.SchDoc

BaseStationBoardMyRev2.SchDoc

BaseStationBoardMyRev3.SchDoc

BaseStationBoardMyRev4.SchDoc

BaseStationBoardMyRev.PcbDoc

BaseStationBoardMyRev2.PcbDoc

BaseStationBoardMyRev3.PcbDoc

BaseStationBoardMyRev4.PcbDoc

\section{Camera Control Board}

CameraBoard.SchDoc

CameraBoardRev2.SchDoc

CameraBoardRev2NewCam.SchDoc

CameraBoard.PcbDoc

CameraBoardRev2.PcbDoc

CameraBoardRev2NewCam.PcbDoc

\section{Main Control Board}

MainControlBoard.SchDoc

MainControlBoardRevision2.SchDoc 
MainControlBoardRevision3.SchDoc

MainControlBoardRevision4.SchDoc

MainControlBoard.PcbDoc

MainControlBoardRevision2.PcbDoc

MainControlBoardRevision3.PcbDoc

MainControlBoardRevision4.PcbDoc

\section{Motor Driver Board}

Motor Driver.SchDoc

Motor Driver.PcbDoc

\section{Camera Test Board}

New Cam Test Board.SchDoc

New Cam Test Board.PcbDoc

\section{Plug Board}

CompleteBoardPlugRevisionBase.SchDoc

CompleteBoardPlugRevisionBase2.SchDoc

MyCompleteBoardPlugRevisionBase.PcbDoc

MyCompleteBoardPlugRevisionBase2.PcbDoc

\section{Power Module Board}

PowerModuleBoardRev1.SchDoc

PowerModuleBoardRev2.SchDoc

PowerModuleBoardRev1.PcbDoc

PowerModuleBoardRev2.PcbDoc 


\section{USB to Serial Board}

USB to Serial.SchDoc

USB to Serial.PcbDoc

\section{SolidWorks Models}

Robot Casing.avi

\section{Boards}

15mmStandOff.SLDPRT

25mmStandOff.SLDPRT

Camera Board Blank.SLDPRT

Camera Board in head.SLDASM

DC-DC Module.SLDPRT

MainBoard.SLDPRT

PlugBoard.SLDPRT

Power Module Board.SLDPRT

Screw.SLDPRT

Stacked Boards.SLDASM

Stacked Boards.SLDDRW

\section{Light Holder}

Assembled.SLDASM

GND_Plate.SLDPRT

PlasticBit.SLDPRT

LightHolder_outer1.SLDPRT

LightHolder_outer2.SLDPRT

LightHolderAssem.SLDASM 
LightHolderAssem.SLDDRW

ScrewIn.SLDPRT

\section{Reel}

Assem1.SLDASM

Frame.SLDPRT

Pivot support.SLDPRT

reel internal.SLDPRT

Reel middle.SLDPRT

reel rotation ring.SLDPRT

Side.SLDPRT

\section{Robot}

AssembledWheel.SLDASM

Body.SLDPRT

Body_CableConnector_LeftSide.SLDPRT

Body_CableConnector_RightSide.SLDPRT

CablePlugConnector_MidSection.SLDPRT

CablePlugConnector_Plug.SLDPRT

CablePlugConnector_RotaryConnection.SLDPRT

Camera_Part_Cam_Holder.SLDPRT

Camera_Part_Cam_Lid.SLDPRT

Camera_Part_Internal1.SLDPRT

Camera_Part_Lid.SLDPRT

Camera_Part_Mid.SLDPRT

Camera_Part_RotationRing.SLDPRT

Camera_Part_SudoBearings.SLDPRT

CameraHead.SLDASM

CameraLens.SLDPRT 
CameraLenseCover.SLDPRT

FullAssembly.SLDASM

Lid With Module.SLDASM

Lid With Stopper.SLDASM

Lid.SLDPRT

Main_Motor.SLDASM

Main_Motor.SLDPRT

Main_Motor_Holder.SLDPRT

Main_Motor_In_Base.SLDASM

Main_Motor_In_Base.SLDDRW

Over_Rotation_Stopper.SLDPRT

PlugAssembled.SLDASM

Shaft.SLDPRT

Tire.SLDPRT

Valve.SLDPRT

Wheel.SLDPRT

Wheel_Shaft_Assemb.SLDASM

WheelRotationCuff.SLDPRT

\section{Other Relevant files}

3rd Edition New Zealand Pipe Inspection Manual.pdf

ENZCON2009 Paper.pdf

ENZCon Presentation.zip 